\title{
Stable twisted curves and their $r$-spin structures
}

\author{
Alessandro Chiodo* \\ September 11, 2018
}

\begin{abstract}
The object of this paper is the notion of $r$-spin structure: a line bundle whose $r$ th power is isomorphic to the canonical bundle. Over the moduli functor $\mathbf{M}_{g}$ of smooth genus- $g$ curves, $r$-spin structures form a finite torsor under the group of $r$-torsion line bundles. Over the moduli functor $\overline{\mathrm{M}}_{g}$ of stable curves, $r$-spin structures form an étale stack, but the finiteness and the torsor structure are lost.

In the present work, we show how this bad picture can be definitely improved simply by placing the problem in the category of Abramovich and Vistoli's twisted curves. First, we find that within such category there exist several different compactifications of $\mathrm{M}_{g}$; each one corresponds to a different multiindex $\vec{l}=\left(l_{0}, l_{1}, \ldots\right)$ identifying a notion of stability: $\vec{l}$-stability. Then, we determine the suitable choices of $\vec{l}$ for which $r$-spin structures form a finite torsor over the moduli of $\vec{l}$-stable curves.
\end{abstract}

\section{Introduction}

For any integer $r \geq 2$, spin structures of order $r$ are natural generalizations of theta characteristics: on a space, they are given by a line bundle $L$ and an isomorphism $f: L^{\otimes r} \stackrel{\sim}{\longrightarrow} \omega$. In this paper we focus on their moduli functor.

For a fixed integer $r \geq 2$, we work over $\operatorname{Spec} \mathbb{Z}[1 / r]$.

\subsection{Smooth curves: the $r$ th roots form a torsor}

For $g \geq 2$ and $2 g-2 \in r \mathbb{Z}$, the category of $r$-spin structures on smooth genus- $g$ curves forms a Deligne-Mumford stack $\mathrm{M}_{g}^{\omega, r}$, finite and étale on $\mathrm{M}_{g}$, which we write as

$$
\mathrm{M}_{g}^{\omega, r}=\left\{(C, L, f) \mid f: L^{\otimes r} \stackrel{\sim}{\longrightarrow} \omega_{C}\right\} / \cong \longrightarrow \mathrm{M}_{g}
$$

In fact $\mathrm{M}_{g}^{\omega, r}$ is a finite torsor under the finite group stack of $r$-torsion line bundles on smooth genus- $g$ curves:

$$
\mathrm{M}_{g}^{\mathcal{O}, r}=\left\{(C, L, f) \mid f: L^{\otimes r} \stackrel{\sim}{\longrightarrow} \mathcal{O}_{C}\right\} / \cong \longrightarrow \mathrm{M}_{g} .
$$

\footnotetext{
${ }^{*}$ Financially supported by the Marie Curie Intra-European Fellowship within the 6th European Community Framework Programme, MEIF-CT-2003-501940.
} 


\subsection{Stable curves: the torsor is lost}

When we extend the study of $r$-spin structures to the category of stable curves $\overline{\mathrm{M}}_{g}$, the properness and the torsor structure are lost.

First, consider the category $\overline{\mathrm{M}}_{g}^{\mathcal{O}, r}$ of $r$-torsion line bundles on stable curves. As above, it forms an étale stack on $\overline{\mathrm{M}}_{g}$ which is equipped with a group structure. However, $\overline{\mathrm{M}}_{g}^{\mathcal{O}, r} \rightarrow \overline{\mathrm{M}}_{g}$ is not proper. Indeed, since $\overline{\mathrm{M}}_{g}^{\mathcal{O}, r}$ is étale and the generic fibre contains $r^{2 g}$ points, one can check that the valuative criterion fails by exhibiting an example of a geometric fibre with less than $r^{2 g}$ points.

1.2.1 Example. Consider an irreducible curve of genus $g$ with only one node. Note that the set of roots of $\mathcal{O}$ consists of $r^{2 g-1}$ elements. Indeed, and more generally, for any stable curve $C$ the group of $r$-torsion line bundles $(\operatorname{Pic} C)_{r}$ fits in the exact sequence

$$
1 \rightarrow \boldsymbol{\mu}_{r} \rightarrow\left(\boldsymbol{\mu}_{r}\right)^{\# V} \rightarrow\left(\boldsymbol{\mu}_{r}\right)^{\# E} \rightarrow(\operatorname{Pic} C)_{r} \rightarrow\left(\operatorname{Pic} C^{\nu}\right)_{r} \rightarrow 1
$$

where $V$ and $E$ are the sets of irreducible components and of singularities of the curve $C$, whereas $C^{\nu}$ is the normalization of $C$. So, we get

$$
\#(\operatorname{Pic} C)_{r}=r^{2 g-1+\#(V)-\#(E)} \text {. }
$$

Second, write $\overline{\mathrm{M}}_{g}^{\omega, r}$ for the category of $r$-spin structures on stable curves. In fact, the morphism to $\overline{\mathrm{M}}_{g}$ is étale; however, it is not proper and it is not a torsor on $\overline{\mathrm{M}}_{g}$. Indeed, the following example shows that the morphism to $\overline{\mathrm{M}}_{g}$ is not surjective.

1.2.4 Example. Let $C$ be a curve with only one node and two irreducible components of genus $g-1$ and 1 , respectively. Then there are no $r$ th roots of $\omega$ on $C$. This happens because the degree of $\omega$ is 1 on the genus- 1 component of $C$. Indeed, recall that the degree of the dualizing sheaf $\omega$ on an irreducible component $C^{\prime}$ of genus $i$ is $2 i-2+\#(N)$ where $N$ is the set of points where $C^{\prime}$ meets the rest of the curve (by "genus" we always mean the arithmetic genus, $\S 2.3$ ).

In the recent years, the interest in moduli of $r$-spin structures has been revived by Witten's conjecture [Wi93], which relates certain enumerative properties of $r$-spin structures to the Gelfand-Dikii hierarchy. The conjecture is a generalization of the Kontsevich-Witten Theorem [Wi91 [Ko92] and has been lately proven in [FSZ]. This result opens the way to further investigations of Gromov-Witten $r$-spin theory.

The original formulation of the relevant enumerative properties was only sketched by Witten in Wi93. A rigourous definition requires - first of all-a compactification of $\mathrm{M}_{g}^{\omega, r}$ (once a suitable compactification is given, the numerical invariants can be defined using [PV01] or [Ch06], see Proposition 4.3.7).

In the existing literature, there are several solutions to the problem of compactifying $\mathrm{M}_{g}^{\omega, r}$ : they consist in enlarging the category $\mathrm{M}_{g}^{\omega, r}$ of smooth $r$-spin curves $(C, L, f)$ to a new category fibred over $\overline{\mathrm{M}}_{g}$. In [Ja98] and [Ja00], Jarvis allows noninvertible sheaves. In [Co87] for $r=2$ and in [CCC07] for all $r$, Cornalba, Caporaso, and Casagrande take as new objects line bundles on semistable curves. In [AJ03. Abramovich and Jarvis realize the same category as in Ja00] in terms of stack-theoretic curves. In all these compactifications the torsor structure is lost over $\overline{\mathrm{M}}_{g}$, because ramification occurs at the new points [Ja00, Thm. 2.4.2] and [CCC07, $\S 3, \S 4.1$. 


\subsection{Placing the problem in the context of twisted curves: $\vec{l}$-stability}

We consider the category $\widetilde{\mathrm{M}}_{g}$ of twisted curves, which are, over an algebraically closed field, stack-theoretic curves whose smooth geometric locus is represented by a scheme and whose stabilizers at the nodes have finite order (see Abramovich and Vistoli AV02 or 2.4 for the definition over a base scheme $X$ ).

Olsson shows that $\widetilde{M}_{g}$ forms an algebraic stack, O107]. However, the stack $\widetilde{M}_{g}$ is nonseparated. Indeed, a twisted curve $\mathrm{C}$ over a discrete valuation ring $R$ with smooth generic fibre $\mathrm{C}_{K}$ is isomorphic to its coarse space over the field of fractions $K$ and may differ from it on the special fibre; in this case the coarse space $|C|$ and the twisted curve $C$ are two nonisomorphic twisted curves extending $C_{K}$ over $R$. Therefore, the valuative criterion of separateness fails.

We describe the condition of stability in the category of twisted curves $\widetilde{M}_{g}$. For any multiindex $\vec{l}=\left(l_{0}, l_{1}, \ldots, l_{\lfloor g / 2\rfloor}\right)$ of invertible integers, we say that a twisted curve is $\vec{l}$-stable if its stabilizers have order $l_{i}$ on nodes of type $i$ (the notion of type of a node can be found in [DM69] and is recalled in (4.1.2)). In this way, for each multiindex $\vec{l}$, we have a notion of stability, which corresponds to a new compactification of $\mathrm{M}_{g}$ (the classical Deligne-Mumford-Knudsen compactification $\overline{\mathrm{M}}_{g}$ corresponds to $\left.\vec{l}=(1, \ldots, 1)\right)$. In the following theorem, we show that the compactifications obtained in this way are all the compactifications of $\mathrm{M}_{g}$ inside $\widetilde{\mathrm{M}}_{g}$.

4.1.4 Theorem. Let us denote by $\mathrm{M}_{g}(\vec{l})$ the category of $\vec{l}$-stable curves. It is contained in $\widetilde{\mathrm{M}}_{g}$ and it contains $\mathrm{M}_{g}$ :

$$
\mathrm{M}_{g} \hookrightarrow \mathrm{M}_{g}(\vec{l}) \hookrightarrow \widetilde{\mathrm{M}}_{g}
$$

I. The stack $\mathrm{M}_{g}(\vec{l})$ is tame, proper (separated), smooth, irreducible and of Deligne-Mumford type. The morphism $\mathrm{M}_{g}(\vec{l}) \rightarrow \overline{\mathrm{M}}_{g}$ is finite, flat, and is an isomorphism on the open dense substack $\mathrm{M}_{g}$.

II. Any proper substack $\mathrm{X}$ of $\widetilde{\mathrm{M}}_{g}$ fitting in $\mathrm{M}_{g} \hookrightarrow \mathrm{X} \hookrightarrow \widetilde{\mathrm{M}}_{g}$ is isomorphic to $\mathrm{M}_{g}(\vec{l})$ for a suitable multiindex $\vec{l}$.

\subsection{The torsor of $r$ th roots of a bundle}

For any line bundle $\mathrm{F}$ on the smooth universal curve on $\mathrm{M}_{g}$ whose relative degree is a multiple of $r$ the category $\mathrm{M}_{g}^{\mathrm{F}, r}$ of $r$ th roots of $\mathrm{F}$ on curves $\mathrm{C} \rightarrow X$ forms a stack, étale and finite on $\mathrm{M}_{g}$, and equipped with a torsor structure under the group stack $\mathrm{M}_{g}^{\mathcal{O}, r}$.

It is well known that $\mathrm{F}$ can be written as a power $\omega^{\otimes k}$ of the relative dualizing sheaf on the universal curve modulo pullbacks from $\mathrm{M}_{g}$ (Enriques and Franchetta's conjecture [Ha83] [Me87] [AC87]). Therefore, in view of an extension of $\mathrm{M}_{g}^{\mathrm{F}, r}$ over $\widetilde{\mathrm{M}}_{g}$, we focus on the case $\mathrm{F}=\omega^{\otimes k}$ and we assume $(2 g-2) k \in r \mathbb{Z}$. We compactify $\mathbf{M}_{g}^{\mathrm{F}, r}$ in two steps:

(1) over $\widetilde{\mathrm{M}}_{g}$, we construct the stack parametrizing $r$ th roots of $\mathrm{F}=\omega^{\otimes k}$;

(2) we restrict such stack to the compactifications $\mathrm{M}_{g}(\vec{l}) \subset \widetilde{\mathrm{M}}_{g}$ for suitable indexes $\vec{l}$.

For (1), we define the stack $\widetilde{\mathrm{M}}_{g}^{\mathrm{F}, r}$ of $r$ th roots of $\mathrm{F}=\omega^{\otimes k}$ on twisted curves

$$
\widetilde{\mathrm{M}}_{g}^{\mathrm{F}, r}=\left\{(\mathrm{C}, \mathrm{L}, \mathrm{f}) \mid \mathrm{f}: \mathrm{L}^{\otimes r} \stackrel{\sim}{\longrightarrow} \mathrm{F}_{\mathrm{C}}\right\} / \cong \longrightarrow \widetilde{\mathrm{M}}_{g}
$$


Note that $\widetilde{\mathrm{M}}_{g}^{\mathrm{F}, r}$ can be regarded as the fibred product $\left(\widetilde{\mathrm{LB}}_{g}\right)_{\mathrm{k}_{r}} \times{ }_{\mathrm{F}} \widetilde{\mathrm{M}}_{g}$, where $\widetilde{\mathrm{LB}}_{g}$ is the stack of line bundles on genus- $g$ twisted curves, $\mathrm{k}_{r}$ is induced by the $r$ th power in $\mathrm{B} \mathbb{G}_{m} \rightarrow \mathrm{B} \mathbb{G}_{m}$, and $\mathrm{F}$ is regarded as a section $\widetilde{\mathrm{M}}_{g} \rightarrow \widetilde{\mathrm{LB}}_{g}$. We show that $\widetilde{\mathrm{M}}_{g}^{\mathrm{F}, r}$ is a Deligne-Mumford stack, étale on $\widetilde{M}_{g}$.

For $(2)$, we choose a multiindex $\vec{l}=\left(l_{0}, l_{1}, \ldots, l_{\lfloor g / 2\rfloor}\right)$ of invertible integers and we consider the restriction $\mathrm{M}_{g}^{\mathrm{F}, r}(\vec{l}) \rightarrow \mathrm{M}_{g}(l)$ of $\widetilde{\mathrm{M}}_{g}^{\mathrm{F}, r} \rightarrow \widetilde{\mathrm{M}}_{g}$. In this way, for each $\vec{l}$, we obtain a stack $\mathrm{M}_{g}^{\mathrm{F}, r}(\vec{l})$ of $r$ th roots of $\mathrm{F}$ on $\vec{l}$-stable curves fibred over $\mathrm{M}_{g}(\vec{l})$. The properness and the torsor structure are lost for general choices of $\vec{l}$ as we already pointed out in the case $\vec{l}=(1, \ldots, 1)$, which corresponds to stable curves. The following theorem determines the suitable choices of $\vec{l}$.

4.2.3 Theorem. For any $\mathrm{F}=\omega^{\otimes k}$, the category $\mathrm{M}_{g}^{\mathrm{F}, r}(\vec{l})$ is a smooth Deligne-Mumford algebraic stack, étale on $\mathrm{M}_{g}(\vec{l})$.

I. For $\mathrm{F}=\mathcal{O}$, the stack $\mathrm{M}_{g}^{\mathcal{O}, r}(\vec{l})$ is a finite group stack if and only if $r$ divides $l_{0}$.

II. For $\mathrm{F}=\omega$ and $2 g-2 \in r \mathbb{Z}$, the stack $\mathrm{M}_{g}^{\mathcal{O}, r}(\vec{l})$ is a finite group stack and $\mathrm{M}_{g}^{\omega, r}(\vec{l})$ is a finite torsor under $\mathrm{M}_{g}^{\mathcal{O}, r}(\vec{l})$ if and only if $r$ divides

$$
(2 i-1) l_{i} \text { for all } i \text {. }
$$

In this way, we obtain several compactifications of the stack $\mathrm{M}_{g}^{\omega, r}$ of smooth $r$-spin curves: for each $\vec{l}$ satisfying $l_{i}(2 i-1) \in r \mathbb{Z}$,

$$
\mathrm{M}_{g}^{\omega, r}(\vec{l}) \rightarrow \mathrm{M}_{g}(\vec{l})
$$

is the finite torsor of $r$-spin $\vec{l}$-stable curves.

III. More generally, for $\mathrm{F}=\omega^{\otimes k}$ and $(2 g-2) k \in r \mathbb{Z}$, the stack $\mathrm{M}_{g}^{\mathcal{O}, r}(\vec{l})$ is a finite group stack and $\mathrm{M}_{g}^{\mathrm{F}, r}(\vec{l})$ is a finite torsor under $\mathrm{M}_{g}^{\mathcal{O}, r}(\vec{l})$ if and only if $r$ divides

$$
l_{0} \quad \text { and }(2 i-1) k l_{i}, \text { for } i>0 .
$$

The fact that these compactifications allow a natural extension of the torsor structure of $r$ th roots defined on the initial uncompactified moduli stack is an improvement in its own right. We further mention some concrete situations in enumerative geometry where this construction is useful:

Gromov-Witten theory. In actual calculations of enumerative geometry of curves, the main advantage of our description of $r$ th roots via this new notion of stability is the generalization to $r$ th roots of the classical tools employed for stable curves. As an example, in [Ch2], we illustrate how the Grothendieck Riemann-Roch formula allows concrete calculations of the genus- $g$ Gromov-Witten invariants of the stack $\left[\mathbb{C}^{2} / G\right]$, where $G$ is a cyclic subgroup of $S L_{2}(\mathbb{C})$. This calculation is the subject of the crepant resolution conjecture, see [BG] for a statement and [CCIT for recent progress in genus 0.

Tensor products of $r$ th roots. In [JKV00, Rem. 4.11] the authors point out that the natural isomorphism $\mathrm{M}_{g}^{\mathrm{F}, r_{1} r_{2}} \cong \mathrm{M}_{g}^{\mathrm{F}, r_{1}} \times \mathrm{M}_{g} \mathrm{M}_{g}^{\mathrm{F}, r_{2}}$, for relatively prime indexes $r_{1}$ and $r_{2}$, does not extend to the boundary. In Proposition 4.2.10 we show that the new compactification allows us to 
extend the equivalence $L \mapsto\left(L^{\otimes r_{2}}, L^{\otimes r_{1}}\right)$ to the boundary over the category of twisted curves with stabilizers of order $r_{1} r_{2}$ on all nodes $\left(r_{1} r_{2}\right.$-stable curves).

In fact, in this way, we get smooth compactifications of the moduli functor of $m$-tuples of spin structures of orders $r_{1}, \ldots, r_{m}$. These moduli stacks are used in [JKV00, Thm. 6.2] in order to describe tensor products of Frobenius manifolds (note that, because of ramifications, taking the fibred product of several Jarvis's compactifications as in [JKV00, §4.2] does not yield a smooth compactification).

Counting boundary points. The description of the geometric points of the boundary locus becomes straightforward: for the indexes $\vec{l}$ defined in the previous theorem, the boundary points are simply represented by $\vec{l}$-stable curves with their $r^{2 g}$ distinct $r$ th roots. We illustrate in Example 4.2.11 that this improves our understanding of the enumerative geometry of $r$ th roots: we show how to count the number of $r$-spin structures on a twisted curve $C$ up to automorphisms of C. This leads to a counterexample of Conjecture 4.2.1 of [Ja01], which states that the Picard group of the moduli stack of smooth genus-1 $r$-spin structures is finite, see Example 4.2.14.

Finally, we point out that the results obtained with previous compactifications extend easily

to the above stacks $\mathrm{M}_{g}^{\mathrm{F}, r}(\vec{l})$. In Proposition 4.3.3, we show that there is a surjective morphism from our compactification to the preexisting compactification due to Abramovich and Jarvis and illustrate where this morphism is not invertible. In Proposition 4.3.7, we prove that the functor of [PV01] and [Ch06] defining the Witten top Chern class yields a class in the rational cohomology of the new compactification and we show that such a class is compatible with previous constructions.

\subsection{Structure of the paper}

In Section 2, we fix our terminology and prove some preliminary results.

In Section 3 we prove the main technical results. The subsection 3.1 is a brief subsection where we prove that the functor of $r$ th roots of a line bundle on a twisted curve on a base scheme $X$ is a Deligne-Mumford stack étale on $X$. The subsection 3.2 focuses on the geometric fibres of this functor on $X$ : we work out the Kummer theory of a twisted curve and compare the long exact Kummer sequence of a twisted curve to that of its coarse space, Theorem 3.2.3. In particular, we state a criterion for a line bundle $\mathrm{F}$ to have $r^{2 g} r$ th roots on a twisted curve, see Theorem 3.2 .2 and see Figure 3.2 at 3.2 .22 ,

By applying these results, in Section 4, we prove Theorem 4.1.4 and Theorem 4.2.3 stated above. We illustrate these theorems in Example 4.2.11. We describe the relation with the previous compactification of Abramovich and Jarvis, Proposition 4.3.3.

In the Appendix we show that line bundles over twisted curves form a stack. A more general treatment which extends to coherent sheaves and proves that such a stack is algebraic can be found in [Li06].

\subsection{Acknowledgements}

I would like to thank Arnaud Beauville, Alessio Corti, Carlos Simpson, Angelo Vistoli, and Charles Walter for comments and help, and especially André Hirschowitz for numerous stimulating discussions and for careful reading of preliminary versions of this paper. 


\section{Terminology and preliminaries}

\section{$2.1 \quad$ Schemes}

We fix an integer $r>0$, and throughout this paper we will consider only schemes over $\operatorname{Spec} \mathbb{Z}[1 / r]$.

\section{$2.2 \quad$ Stacks}

Terminology and generalities. Our general reference is [LM00]. An algebraic stack is a stack satisfying Artin's definition [Ar74]. Stacks in the sense of Deligne and Mumford [DM69] will be called Deligne-Mumford stacks. When working with algebraic stacks with finite diagonal, we use Keel and Mori's Theorem [KM97]: there exists an algebraic space $|X|$ associated to the stack $\mathrm{X}$ and a morphism $\pi_{\mathrm{X}}: \mathrm{X} \rightarrow|\mathrm{X}|$ (or simply $\pi$ ) which is universal with respect to morphisms from $X$ to algebraic spaces. We refer to $|X|$ as the coarse space. In this way we have a functor (and in fact a 2-functor) associating to any morphism between this type of stacks $\mathrm{f}: \mathrm{X} \rightarrow \mathrm{Y}$ the unique morphism between the corresponding coarse algebraic spaces $|\mathrm{f}|:|\mathrm{X}| \rightarrow|\mathrm{Y}|$ satisfying $\mathrm{f} \circ \pi_{Y}=\pi_{X} \circ|\mathrm{f}|$.

We refer to [Br90] for the notion of group stack $G \rightarrow X$. We say that there is an action of the group stack $\mathrm{G} \rightarrow \mathrm{X}$ with product $\mathrm{m}_{\mathrm{G}}$ and unit object e on $\mathrm{T} \rightarrow \mathrm{X}$ if there is a morphism of stacks $\mathrm{m}: \mathrm{G} \times \mathrm{X} T \rightarrow \mathrm{T}$ and homotopies $\mathrm{m} \circ\left(\mathrm{m}_{\mathrm{G}} \times \mathrm{id}_{\mathrm{T}}\right) \Rightarrow \mathrm{m}\left(\mathrm{id}_{\mathrm{G}} \times \mathrm{m}\right)$ and $\mathrm{m} \circ\left(\mathrm{e} \times \mathrm{id}_{\mathrm{T}}\right) \Rightarrow \mathrm{id}_{\mathrm{T}}$ satisfying the associativity constraint [Br90, 6.1.3] and the compatibility constraint [Br90, 6.1.4]. The morphism $\mathrm{T} \rightarrow \mathrm{X}$ is a torsor if the morphism

$$
\mathrm{m} \times \mathrm{pr}_{2}: \mathrm{G} \times \mathrm{x} \mathrm{T} \rightarrow \mathrm{T} \times \mathrm{x} \mathrm{T}
$$

is an isomorphism of stacks and $\mathrm{T} \rightarrow \mathrm{X}$ is flat and surjective.

Morphisms of stacks. We often need to consider 2-categories in which the objects are algebraic stacks, the functors between two stacks are regarded as 1-morphisms, and the natural transformations are regarded as 2-morphisms.

The situation is often simplified by the following criterion showing that certain morphisms between stacks have only trivial 2-automorphisms. In particular this criterion applies to morphisms between twisted curves.

2.2.1 Lemma (Abramovich and Vistoli, [AV02, Lem. 4.2.3]). Let $\mathrm{f}: \mathrm{X} \rightarrow \mathrm{Y}$ be a representable morphism of Deligne-Mumford stacks over a scheme $S$. Assume that there exists a dense open representable substack (i.e. an algebraic space) $U \subset \mathrm{X}$ and an open representable substack $V \subset Y$ such that $\mathrm{f}$ maps $U$ into $V$. Further assume that the diagonal $\mathrm{Y} \rightarrow \mathrm{Y} \times_{S} \mathrm{Y}$ is separated. Then any automorphism of $\mathrm{f}$ is trivial.

Stabilizer of a geometric point of a stack. Let $X$ be an algebraic stack. A geometric point $\mathrm{p} \in \mathrm{X}$ is an object Spec $k \rightarrow \mathrm{X}$, where $k$ is algebraically closed. We denote by Aut(p) the automorphism group of $p$ as an object of the fibred category $X_{p}$. We refer to Aut(p) as the stabilizer of $\mathrm{p}$. 
Local pictures. We often need to describe stacks and morphisms between stacks locally in terms of explicit equations. We adopt the following standard convention, which avoids repeated mention of strict henselization [ACV03, \$1.5].

Let $\mathrm{X}$ and $\mathrm{U}$ be algebraic stacks and let $\mathrm{x} \in \mathrm{X}$ and $\mathrm{u} \in \mathrm{U}$ be geometric points. We say "the local picture of $X$ at $x$ is given by $U$ (at $u$ )" if there is an isomorphism between the strict henselization $X^{\text {sh }}$ of $X$ at $x$ and the strict henselization $U^{\text {sh }}$ of $U$ at $u$.

If $\mathrm{f}: \mathrm{X} \rightarrow \mathrm{Y}$ and $\mathrm{g}: \mathrm{U} \rightarrow \mathrm{V}$ are morphisms of stacks and $\mathrm{x}$ and $\mathrm{u}$ are geometric points in $X$ and $U$, we say "the local picture of $X \rightarrow Y$ at $x$ is given by $U \rightarrow V$ (at $u$ )" if there is an isomorphism between the strict henselization $f^{\text {sh }}: X^{\text {sh }} \rightarrow Y^{\text {sh }}$ of $f$ at $x$ and the strict henselization $\mathrm{g}^{\text {sh }}: \mathrm{U}^{\mathrm{sh}} \rightarrow \mathrm{V}^{\mathrm{sh}}$ of $\mathrm{g}$ at $\mathrm{u}$. This convention allows local descriptions of diagrams of morphisms between stacks; in particular it allows local description of group actions $G \times \mathrm{X} \rightarrow \mathrm{X}$ and of $G$-equivariant morphisms.

The construction of Cadman, Matsuki, Olsson, and Vistoli. For any smooth scheme $X$ and smooth effective divisor $D$ in $X$ we present a stack-theoretic modification of $X$, which contains $X \backslash D$ as a dense open representable substack and has coarse space $X$.

In Ca07, Cadman provides the following construction.

2.2.2 Definition. Let $X$ be a smooth scheme $X$, let $D$ be a smooth effective Cartier divisor in $X$, and let $l$ be a positive integer invertible on $X$. The category $X[D / l]$ is formed by objects $(S, M, j, s)$, where

1. $S$ is an $X$-scheme $S \rightarrow X$;

2. $M$ is a line bundle on $S$;

3. $j$ is an isomorphism between $M^{\otimes l}$ and the pullback of $\mathcal{O}(D)$ on $S$;

4. $s$ is a section $s \in \Gamma(S, M)$ such that $j\left(s^{\otimes l}\right)$ equals the tautological section of $\mathcal{O}(D)$ vanishing along $D$.

The morphisms are defined in the obvious way.

This definition yields a Deligne-Mumford stack $X[D / l]$, with coarse space $X$. The morphism $\pi: X[D / l] \rightarrow X$ is an isomorphism over $X \backslash D$ : we have

$$
X \backslash D \hookrightarrow X[D / l] \stackrel{\pi}{\longrightarrow} X
$$

where $X \backslash D$ is dense in $X[D / l]$ and $\pi$ is finite and flat. Note that $X[D / l]$ is equipped with a tautological line bundle $\mathrm{M}$ and an isomorphism

$$
\mathrm{M}^{\otimes l} \stackrel{\sim}{\longrightarrow} \pi^{*} \mathcal{O}_{X}(D)
$$

A special case of this construction was first introduced by Abramovich, Graber, and Vistoli [AGV01, 3.5.3] (the idea is attributed to Vistoli, see [Ab, 3.5]). In the existing literature, two different and compatible definitions can be found: see Matsuki and Olsson [MO05] and Cadman [Ca07] for the above definition (see [Ca07, 2.4.5] for the compatibility between the two constructions).

It is natural to try and generalize this construction. If $D_{1}, \ldots, D_{n}$ are distinct smooth effective divisors with normal crossings, for any positive integers $l_{1}, \ldots, l_{n}$ invertible on $X$, we 
write

$$
X\left[D_{1} / l_{1}+\cdots+D_{n} / l_{n}\right]:=X\left[D_{1} / l_{1}\right] \times_{X} \cdots \times_{X} X\left[D_{n} / l_{n}\right] .
$$

In this way, $X\left[D_{1} / l_{1}+\cdots+D_{n} / l_{n}\right]$ is a smooth Deligne-Mumford stack.

2.2.5 Generalization. The definition provided in MO05 by Matsuki and Olsson generalizes the above definition of $X\left[D_{1} / l_{1}+\cdots+D_{n} / l_{n}\right]$ to the case where singular divisors $D_{i}$ occur, and the local picture of $D_{i}$ at each point is the union of smooth divisors with normal crossings (a normal crossings divisor). This extension involves the notion of logarithmic structures in the sense of Fontaine and Illusie or the use of étale descent. The output is again a smooth stack, see [MO05, Thm. 4.1].

2.2.6 Example. Consider the affine space $X=\operatorname{Spec} R$ and the divisor $D=\{t=0\}$ for $t \in R$. Assume that $l$ is invertible in $R$. The stack $X[D / l]$ is the quotient stack $\left[\operatorname{Spec} \widetilde{R} / \boldsymbol{\mu}_{l}\right]$, where $\widetilde{R}=\operatorname{Spec} R[\widetilde{t}] /(\widetilde{t}-t)$ and $\boldsymbol{\mu}_{l}$ acts on $\widetilde{R}$ as $g \cdot \widetilde{t}=g^{-1} \widetilde{t}$ and fixes $R$.

2.2.7 Example. Let $\mathrm{X}$ be a Deligne-Mumford stack, whose coarse space is a proper, regular, and reduced curve $|\mathrm{X}|$ over a field $k$ and whose geometric points have trivial stabilizers except for a finite number of distinct points $\mathrm{p}_{1}, \ldots, \mathrm{p}_{n}$ with stabilizers of order $l_{1}, \ldots, l_{n}$ (which we assume invertible). In that case, we have (see [Ca07, Exa. 3.7])

$$
\mathbf{X} \cong|\mathbf{X}|\left[p_{1} / l_{1}+\cdots+p_{n} / l_{n}\right]
$$

where $p_{i}=\left|\mathrm{p}_{i}\right| \in|\mathrm{X}|$ is the point corresponding to $\mathrm{p}_{i} \in \mathrm{X}$. The local picture at $\mathrm{p}_{i}$ : Spec $k \rightarrow \mathrm{X}$ is given by $S\left[0 / l_{i}\right]$, where $S=\operatorname{Spec} k[z]$ and 0 denotes $\{z=0\}$. In this way there is an isomorphism between $\operatorname{Aut}\left(\mathrm{p}_{i}\right)$ and cyclic group of $l_{i}$ th roots of unity of $\mathbb{G}_{m}$.

2.2.8 Remark. At a point $\mathrm{p}_{i}: \operatorname{Spec} k \rightarrow \mathrm{X}=|\mathrm{X}|\left[p_{1} / l_{1}+\cdots+p_{n} / l_{n}\right]$ the projection $\mathrm{pr}_{2}$ from Spec $k_{\left|\mathrm{p}_{i}\right|} \times_{\pi} \mathrm{X}$ to $\mathrm{X}$ induces a canonical embedding $\mathrm{j}_{i}: \mathrm{B}\left(\operatorname{Aut}\left(\mathrm{p}_{i}\right)\right) \hookrightarrow \mathrm{X}$. The group Pic(B(Aut $\left.\left.\mathrm{p}_{i}\right)\right)$ is a cyclic group of order $l_{i}$. The tangent space $\mathrm{T}_{i}$ at $\mathrm{p}_{i}$ is a representation of $\operatorname{Aut}\left(\mathrm{p}_{i}\right)$ and is a canonical generator of $\operatorname{Pic}\left(\right.$ Aut $\left.p_{i}\right)$. In this way $\operatorname{Pic}\left(\right.$ Aut $\left.p_{i}\right)$ is canonically isomorphic to $\mathbb{Z} / l_{i} \mathbb{Z}$.

2.2.9 Remark. On a Deligne-Mumford stack of dimension 1, the degree of a line bundle $\mathrm{F}$ is the degree of the first Chern class $c_{1}(\mathrm{~F})$ in the rational Chow ring $\in A^{1}(\mathrm{X})_{\mathbb{Q}}$. Therefore $\operatorname{deg}(\mathrm{F})$ is a rational number, see [Kr99, 3.3] and references therein. In the case of $C[p / d]$, we have the following proposition.

2.2.10 Proposition. Let $C$ be a proper, regular, and reduced curve over a field $k$. For a closed point $p \in C$ and $l$ a positive integer, consider $\pi: C[p / l] \rightarrow C$. We write $\Gamma$ for the stabilizer of the point of $C[p / l]$ over $p$ and write $\mathrm{j}: \mathrm{B} \Gamma \rightarrow C[p / d]$ for the canonical embedding of Remark 2.2.8. For any line bundle $\mathrm{F}$ on $C[p / d]$, the degree of $\mathrm{F}$ belongs to $\frac{1}{l} \mathbb{Z}$ and we have

$$
l \operatorname{deg}(\mathrm{F}) \equiv \mathrm{j}^{*}(\mathrm{~F}) \in \mathbb{Z} / l \mathbb{Z}
$$

where we used the canonical identification of $\mathrm{Pic}(\mathrm{B \Gamma})$ with $\mathbb{Z} / l \mathbb{Z}$ of Remark 2.2.8.

Proof. Let $h \in\{0, \ldots, l-1\}$ be the integer satisfying $\mathrm{j}^{*}(\mathrm{~F})=\mathrm{T}^{\otimes h}$, for $\mathrm{T}$ the tangent space at $\mathrm{p}$. The tautological line bundle $\mathrm{M}$ on $C[p / d]$ satisfies $\mathrm{M}^{\otimes l} \cong \pi^{*} \mathcal{O}_{C}(p),(2.2 .3)$. Note that $\mathrm{j}^{*} \mathrm{M}=\mathrm{T}$. 
Hence, $\mathrm{F} \otimes\left(\mathrm{M}^{\otimes h}\right)^{\vee}=\pi^{*} \mathcal{O}_{C}(D)$ for an integral divisor $D$ on $C$, and we have

$$
\begin{aligned}
l \operatorname{deg}(\mathrm{F}) & =l \operatorname{deg}\left(\mathrm{M}^{\otimes h} \otimes \pi^{*} \mathcal{O}_{C}(D)\right) \\
& =\operatorname{deg}\left(\mathrm{M}^{\otimes h l} \otimes \pi^{*} \mathcal{O}_{C}(l D)\right) \\
& =\operatorname{deg}\left(\pi^{*} \mathcal{O}(h[p]) \otimes \pi^{*} \mathcal{O}_{C}(l D)\right) \\
& =\operatorname{deg}_{C}\left(\mathcal{O}(h[p]) \otimes \mathcal{O}_{C}(l D)\right) \\
& =h+l \operatorname{deg} D \\
& \equiv h \quad \bmod l \\
& \equiv j^{*}(\mathrm{~F}) \quad \bmod l .
\end{aligned}
$$

\subsection{Stable curves}

We recall some standard definition for sake of clarity and for preparing the definition of twisted curve in $§ 2.4$. For any integer $g \geq 2$, a stable curve of genus $g$ on a scheme $X$ is a proper and flat morphism $C \rightarrow X$ satisfying the following conditions:

1. each geometric fibre $C_{x}$ over $x$ in $X$ is reduced, connected, has dimension one, and has only ordinary double points (which we usually call nodes);

2. the dualizing sheaf of $C_{x}$ is ample;

3. $\operatorname{dim}_{k(x)} H^{1}\left(C_{x}, \mathcal{O}_{C_{x}}\right)=g$.

A stable curve $C$ over an algebraically closed field $k$ yields a natural combinatorial object: the dual graph. The dual graph $\Lambda$ of $C$ is the graph whose set of vertices $V$ is the set of irreducible components of $C$ and whose set of edges $E$ is the set of nodes of $C$. An edge connects the vertices corresponding to the irreducible components containing the two branches of the nodes. If an orientation of $\Lambda$ is fixed, we have a chain complex $\mathcal{C}_{\bullet}(\Lambda, \mathbb{Z} / r \mathbb{Z})$ with differential

$$
\partial:(\mathbb{Z} / r \mathbb{Z})^{E} \rightarrow(\mathbb{Z} / r \mathbb{Z})^{V}
$$

where the edge starting at $v_{-}$and ending at $v_{+}$is sent to the 0 -chain $\left[v_{+}\right]-\left[v_{-}\right]$. We say that a node $e$ of a stable curve $C$ is separating if by normalizing $C$ at the point $e$ we obtain two disjoint components.

If we assign to each vertex $v \in V$ the genus $g_{v}$ of the connected component of the normalization of $C$ corresponding to the irreducible component attached to $v$, the (arithmetic) genus of $C$ can be read off from $\Lambda$ and the function $v \mapsto g_{v}$. Indeed, we have

$$
g(C)=b_{1}+\sum_{v} g_{v}
$$

where $b_{1}=1-\#(V)+\#(E)$ is the first Betti number of $\Lambda$.

\subsection{Twisted curves}

We recall the notion of twisted curve due to Abramovich and Vistoli, see [AV02] or the remarks below for some slight generalizations. 
2.4.1 Definition. A twisted curve of genus $g$ on a scheme $X$ is a proper and flat morphism of tame stacks $\mathrm{C} \rightarrow X$, for which

1. the fibres are purely 1-dimensional with at most nodal singularities,

2. the coarse space is a stable curve $|\mathrm{C}| \rightarrow X$ of genus $g$;

3. the smooth locus $\mathrm{C}^{\mathrm{sm}}$ is an algebraic space;

4. the local picture at a node is given by $\left[U / \boldsymbol{\mu}_{l}\right] \rightarrow T$, where

- $T=\operatorname{Spec} A$,

- $U=\operatorname{Spec} A[z, w] /(z w-t)$ for some $t \in A$, and

- the action of $\boldsymbol{\mu}_{l}$ is given by $(z, w) \mapsto\left(\xi_{l} z, \xi_{l}^{-1} w\right)$.

(Recall that the tameness condition on $\mathrm{C}$ means that for every geometric point $\mathrm{p}:$ Spec $k \rightarrow \mathrm{C}$ the group Aut(p) has order prime to the characteristic of the algebraically closed field $k$.)

2.4.2 Remark (unbalanced twisted curves). In the existing literature twisted curves satisfying the above local condition (4) are often called balanced, AV02. We drop the adjective balanced, because we never consider unbalanced twisted curves.

2.4.3 Remark (twisted curves with smooth stack-theoretic points). A further natural generalization, which is usually given in the definition of a twisted curve (see [AV02, Def. 4.1.2]), consists of allowing nontrivial stabilizers on smooth points. By [Ca07, Thm 4.1] and [O107, Thm 1.8], giving a stack-theoretic curve with a smooth point whose stabilizer has order $l$ is equivalent to assigning an invertible integer $l$, a twisted curve $C \rightarrow X$ in the sense of Definition 2.4.1, and a section $\sigma: X \rightarrow \mathrm{C}$ in the smooth locus. (With the notation introduced above, the equivalence is essentially $(\mathrm{C}, \sigma, l) \mapsto \mathrm{C}[\sigma(X) / l]$.)

2.4.4 Remark (unstable coarse space). Twisted curves are generally defined without imposing the condition of stability on the coarse space. In Olsson's paper [0107], it is shown that these stack-theoretic curves form an algebraic stack in the sense of Artin's definition. If we require that the coarse space $|C|$ is stable, we get a Deligne-Mumford stack. See Theorem 2.4.6.

A 1-morphism $\mathrm{C} \rightarrow X$ to $\mathrm{C}^{\prime} \rightarrow X^{\prime}$ between twisted curves is a morphism of stacks $\mathrm{m}$ fitting in the fibre diagram

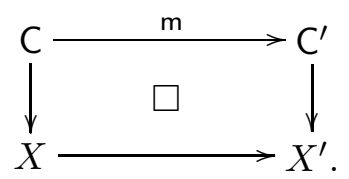

By Lemma 2.2.1, there is at most one natural transformation, which identifies two morphisms. Therefore, we obtain a category by considering morphisms as 1-morphisms up to base-preserving natural transformations.

In [O107, Thm. 1.9], Olsson proves that such a category is an algebraic stack. In particular, see [0107, Rem. 1.10], he shows how the versal deformation space of a twisted curve $C$ over a field $k$ relates to the versal deformation space $\operatorname{Spec} I$ of the coarse space $|C|$. Let $\mathrm{e}_{1}, \ldots, \mathrm{e}_{m}$ be the nodes of $C$ and $\left|e_{1}\right|, \ldots,\left|e_{m}\right|$ the corresponding nodes of $|C|$. Let $l_{i}$ be the order of the automorphism group of $\mathrm{e}_{i}$. Let

$$
D_{i}=\left\{t_{i}=0\right\}
$$

be the divisor classifying deformations of $|C|$ on which the node $\left|e_{i}\right|$ persists: 
2.4.6 Theorem (Olsson, O107, Thm. 1.9]). The category of twisted curves $\widetilde{\mathrm{M}}_{g}$ in the sense of Definition 2.4.1 is a smooth Deligne-Mumford stack and the versal deformation space of a twisted curve $\mathrm{C} \rightarrow$ Spec $k$ is given by

$$
\widetilde{I}:=I\left[z_{1}, \ldots, z_{m}\right] /\left(z_{1}^{l_{1}}-t_{1}, \ldots, z_{m}^{l_{m}}-t_{m}\right),
$$

where $t_{1}, \ldots, t_{m} \in I$ are the parameters satisfying (2.4.5) and $l_{1}, \ldots, l_{m}$ are the orders of the stabilizers of the corresponding nodes.

The group of automorphisms of a twisted curve acts naturally on the versal deformation space of a twisted curve. Using [ACV03, we describe the automorphisms of a twisted curve. Then, in Remark 2.4.10, we illustrate the action on the versal deformation.

Automorphisms of twisted curves. Let $\pi: C \rightarrow|C|$ be a twisted curve over an algebraically closed field $k$. In [ACV03, Prop. 7.1.1] the group $\operatorname{Aut}(\mathrm{C},|\mathrm{C}|)$ of automorphisms of $\mathrm{C}$ that fix the coarse space $|C|$ is explicitly calculated:

2.4.8 Theorem (Abramovich, Corti, Vistoli, [ACV03, Prop. 7.1.1]). For a twisted curve over an algebraically closed field, we have an isomorphism

$$
\operatorname{Aut}(\mathrm{C},|\mathrm{C}|) \cong \boldsymbol{\mu}_{l_{1}} \times \cdots \times \boldsymbol{\mu}_{l_{m}}
$$

where $l_{1}, \ldots, l_{m}$ are the orders of the stabilizers at the nodes $\mathrm{e}_{1}, \ldots, \mathrm{e}_{m}$.

2.4.9 Remark. In fact [ACV03, Thm. 7.1.1] shows that we can choose generators $\mathrm{g}_{1}, \ldots, \mathrm{g}_{m}$ of $\operatorname{Aut}(\mathrm{C},|\mathrm{C}|)$ such that the restriction of $\mathrm{g}_{i}$ to $\mathrm{C} \backslash\left\{\mathrm{e}_{i}\right\}$ is the identity, and the local picture at $\mathrm{e}_{i}$ is given by

$$
\begin{aligned}
k[z, w] /(z w) & \rightarrow k[z, w] /(z w) \\
(z, w) & \mapsto\left(z, \xi_{l_{i}} w\right),
\end{aligned}
$$

where $\xi_{l_{i}}$ is a primitive $l_{i}$ th root of unity. (Local pictures are given up to natural transformations, and the 1-automorphism above is in fact, locally on the strict henselization, 2-isomorphic to $(z, w) \mapsto\left(\xi_{l_{i}}^{a} z, \xi_{l_{i}}^{b} y\right)$ for any $a, b \in\left\{0, \ldots, l_{i}-1\right\}$ satisfying $\left.a+b=1 \bmod l_{i}.\right)$

2.4.10 Remark. The group Aut $(\mathrm{C},|\mathrm{C}|)$, with the basis given above, acts on the versal deformation space (2.4.7) as $\left(\mathrm{g}_{1}, \ldots, \mathrm{g}_{m}\right) z_{i}=\xi_{l_{i}} z_{i}$ (see [0107, Lem. 5.3]).

\subsection{Line bundles on twisted curves}

In view of the study of $r$ th roots in the Picard group we review some facts on line bundles on twisted curves.

2.5.1 Proposition. For any twisted curve $\mathrm{f}: \mathrm{C} \rightarrow X$ with coarse space $|\mathrm{f}|:|\mathrm{C}| \rightarrow X$ the pullback via $\pi: \mathrm{C} \rightarrow|\mathrm{C}|$ of the relative dualizing sheaf of $|\mathrm{f}|$ is the relative dualizing sheaf of $\mathrm{f}$.

Proof. The natural homomorphism $\pi^{*} \omega_{|\mathrm{f}|} \rightarrow \omega_{\mathrm{f}}$ is an isomorphism on the smooth locus. At the 
nodes the local picture of $\mathrm{C} \rightarrow|\mathrm{C}|$ is

$$
\begin{aligned}
U=\operatorname{Spec} A[z, w] /(z w-t) & \rightarrow V=\operatorname{Spec} A[x, y] /\left(x y-t^{l}\right) \\
(z, w) & \mapsto\left(z^{l}, w^{l}\right)
\end{aligned}
$$

with the stabilizer of the node $\boldsymbol{\mu}_{l}$ acting as $\xi_{l} \cdot(z, w)=\left(\xi_{l} z, \xi_{l}^{-1} w\right)$. The local generator of the relative dualizing sheaf of $|\mathrm{f}|$ is

$$
\frac{d z^{l}}{z^{l}}-\frac{d w^{l}}{w^{l}}
$$

and is sent to the local generator $d z / z-d w / w$ of $\omega_{f}$ (recall that $d\left(z^{l}\right)=l z^{l-1} d z$ and $d\left(w^{l}\right)=$ $\left.l w^{l-1} d w\right)$.

We now fix a twisted curve over an algebraically closed field $k$ and describe the action by pullback of $\operatorname{Aut}(\mathrm{C})$ on $\operatorname{Pic}(\mathrm{C})$. We take one of the automorphisms belonging to $\operatorname{Aut}(\mathrm{C}, \mathrm{C} \backslash\{\mathrm{e}\}) \subseteq$ $\operatorname{Aut}(\mathrm{C},|\mathrm{C}|)$ fixing the entire twisted curve away from a node e with stabilizer of order $l$. We describe explicitly such automorphism by choosing for the rest of this section a primitive $l$ th root of unity $\xi$. The local picture of $C$ at e is given by $\left[V / \boldsymbol{\mu}_{l}\right]$, where $V$ equals $\left\{z_{+} z_{-}=0\right\}$ and $\boldsymbol{\mu}_{l}$ acts as $\left(z_{+}, z_{-}\right) \mapsto\left(\xi z_{+}, \xi^{-1} z_{-}\right)$. The automorphism $\mathrm{g} \in \operatorname{Aut}(\mathrm{C},|\mathrm{C}|)$ is chosen in such a way that $C \backslash\{\mathrm{e}\}$ is fixed and the local picture at $\mathrm{e}$ is

$$
\mathrm{g}^{\mathrm{sh}}:\left(z_{+}, z_{-}\right) \mapsto\left(z_{+}, \xi z_{-}\right) .
$$

We already noted in Theorem 2.4.8 and in Remark 2.4.9 that all automophisms of $\mathrm{C}$ are equal (up to natural transformation) to the composite of pullbacks from $|\mathrm{C}|$ and products of morphisms defined in the same way as $\mathrm{g}$.

We now take a line bundle $L$ on $C$ and show in Proposition 2.5.3 that pulling back $L$ via $g$ is the same as tensoring $L$ by a line bundle $T_{L}$ in the torsion subgroup of the Picard group of $C$. We need to set up some standard notation identifying the line bundle $T_{L}$.

Consider the homomorphism

$$
\gamma: \mathbb{G}_{m} \rightarrow \operatorname{Pic} \mathrm{C}
$$

sending $\lambda \in \mathbb{G}_{m}$ to the line bundle of regular functions $f$ on the partial normalization at the node e satisfying $f\left(\mathrm{p}_{+}\right)=\lambda f\left(\mathrm{p}_{-}\right)$, where $\mathrm{p}_{+}$and $\mathrm{p}_{-}$are the points of the normalization lifting the node e.

Consider the pullback of $L$ on the partial normalization, and its restriction on $B\left(\operatorname{Aut}\left(p_{+}\right)\right)$. We already noted in Remark 2.2.8 that the Picard group of $B\left(\operatorname{Aut}\left(\mathrm{p}_{+}\right)\right)$is canonically generated by $T_{p_{+}}$. Therefore, $L$ determines on $B\left(\operatorname{Aut}\left(p_{+}\right)\right)$a power of $T_{p_{+}}$; we denote its exponent by

$$
\text { mult }_{\mathbf{p}_{+}} \mathrm{L} \in\{0, \ldots, l-1\} .
$$

Note that $\gamma$ and mult $p_{p_{+}} \mathrm{L}$ are transformed into $\lambda \mapsto \gamma\left(\lambda^{-1}\right)$ and into $l-$ mult $_{\mathbf{p}_{+}} \mathrm{L}$ (reduced modulo $l$ ) if we interchange the notations $\mathrm{p}_{+}$and $\mathrm{p}_{-}$. This implies that the line bundle

$$
\mathrm{T}_{\mathrm{L}}=\gamma\left(\xi^{\text {mult }_{\mathrm{p}_{+}} \mathrm{L}}\right) \in \operatorname{Pic}(\mathrm{C})
$$

only depends on $L$ and does not depend on the notation $p_{+}, p_{-}$. 
2.5.3 Proposition. For any line bundle $\mathrm{L}$ on $\mathrm{C}$ over an algebraically closed field $k$ and for $\mathrm{g} \in \operatorname{Aut}(\mathrm{C}, \mathrm{C} \backslash\{\mathrm{e}\})$ satisfying (2.5.2) we have

$$
g^{*} L \cong L \otimes T_{L}
$$

Proof. The local picture of $\mathrm{L} \rightarrow \mathrm{C}$ at the point of the zero section over e is

$$
\mathrm{W}=\left[\left(V \times \mathbb{A}^{1}\right) / \boldsymbol{\mu}_{l}\right] \rightarrow\left[V / \boldsymbol{\mu}_{l}\right]
$$

where $V$ is $\left\{z_{+} z_{-}=0\right\}$ as above, and $\xi \in \operatorname{Aut}(\mathrm{e}) \operatorname{acts}$ on $\left(\left(z_{+}, z_{-}\right), \lambda\right) \in V \times \mathbb{A}^{1}$ as

$$
\left(\left(z_{+}, z_{-}\right), t\right) \mapsto\left(\left(\xi z_{+}, \xi^{-1} z_{-}\right), \xi^{\text {mult } \left._{\mathrm{p}_{+}} \mathrm{L}_{t}\right)}\right.
$$

By tensoring with a suitable element of $\pi^{*} \mathrm{Pic}|\mathrm{C}|$, we can restrict to the case of a line bundle $\mathrm{L}$ on $\mathrm{C}$ which is trivial on $\mathrm{C} \backslash\{\mathrm{e}\}$. In this way, for $V^{\times}=V \backslash(0,0)$, we can regard $\mathrm{L}$ as the datum of a line bundle $\mathrm{W}$ on $\left[V / \boldsymbol{\mu}_{l}\right]$ alongside with an isomorphism $\Phi$ between $\left.\mathrm{W}\right|_{V^{\times}}$and the submodule of $\mathcal{O}_{V^{\times}}=k\left[z_{+}, z_{+}^{-1}\right] \oplus k\left[z_{-}, z_{-}^{-1}\right]$ invariant under the action of $\boldsymbol{\mu}_{l}$. By (2.5.5), the line bundle $\mathrm{W}$ is trivial on $V$ and $\boldsymbol{\mu}_{l}$-linearized by the character $\xi \mapsto \xi^{h}$ for $h=$ mult $_{\mathrm{p}_{+}} \mathrm{L}$. Note that invariant sections of $\left.\mathrm{W}\right|_{V^{\times}}$form a module $z_{+}^{l-h} k\left[z_{+}^{l}, z_{+}^{-l}\right] \oplus z_{-}^{h} k\left[z_{-}^{l}, z_{-}^{-l}\right]$. Pulling back via $\mathrm{g}:\left(z_{+}, z_{-}\right) \mapsto\left(z_{+}, \xi z_{-}\right)$changes $\Phi$ by multiplication by $\left(1 \oplus \xi^{h}\right)$ in $k\left[z_{+}, z_{+}^{-1}\right] \oplus k\left[z_{-}, z_{-}^{-1}\right]$. In this way, we only change the descent datum along the partial desingularization at e by multiplication by $\xi^{h}$ on the branch $\left(z_{+}=0\right)$. This amounts to tensoring as in (2.5.4).

On a stable curve $C \rightarrow$ Spec $k$, the total degree of a line bundle is the sum of the degrees on the connected components of the normalization. We extend this definition to twisted curves.

2.5.6 Proposition. The total degree of a line bundle $\mathrm{F}$ on a twisted curve $\mathrm{C}$ over $k$ is an integer.

Proof. We only need to prove the claim in the case when $F$ is trivial on $C \backslash\{\mathrm{e}\}$ and nontrivial on the node e. Then, in order to calculate the total degree, we can regard $F$ as a line bundle on a twisted curve $C$ with trivial stabilizers on every node except e. The normalization of $C$ is a smooth stack $C^{\nu}$ as in Example 2.2.7 $C^{\nu}=X\left[p_{1} / l+p_{2} / l\right]$ for $p_{1}, p_{2} \in X$ and $l \in \mathbb{Z}_{\geq 1}$. We calculate the total degree using Proposition 2.2.10.

First, assume that the partial normalization of $C$ at e is the disconnected stack $C^{\nu}=D_{1} \sqcup D_{2}$. By Proposition 2.2.10, the total degree of $\mathrm{F}$ is in $\frac{1}{l} \mathbb{Z}$. We need to show that the total degrees of the restrictions $F_{1}$ and $F_{2}$ on $D_{1}$ and $D_{2}$ satisfy

$$
l \operatorname{deg}\left(\mathrm{F}_{1}\right)+l \operatorname{deg}\left(\mathrm{F}_{2}\right) \equiv 0 \quad \bmod l .
$$

By Proposition 2.2.10, this amounts to showing that the pullbacks of $F_{i}$ with respect to B Aut $\left(\mathrm{p}_{i}\right) \rightarrow \mathrm{D}_{i}$ yield inverse characters of for $i=1$ and 2 . This follows using the local picture given above, (2.5.5).

Finally, if the partial normalization $\mathrm{h}: \mathrm{C}^{\nu} \rightarrow \mathrm{C}$ is connected we have two distinct points $\mathrm{p}_{1}$ and $\mathrm{p}_{2}$ with nontrivial stabilizer of order $d$ lying over e. We can define two line bundles $\mathrm{F}_{1}$ and 
$\mathrm{F}_{2}$ on $\mathrm{C}^{\nu}$ such that $\mathrm{F}_{i}$ is trivial at $\mathrm{p}_{i}$, and $\mathrm{F}_{1} \otimes \mathrm{F}_{2}=\mathrm{h}^{*} \mathrm{~F}$. Then, (2.5.7) holds (with the same proof) and this implies the claim.

\section{The functor of $r$ th roots of a line bundle}

This section is divided in two subsections: 93,1 and 93,2 .

1. Relying on the results of [Li06] or on the appendix, this first part recalls briefly that $r$ th roots of a line bundle form a Deligne-Mumford stack. We consider a twisted curve $\mathrm{C} \rightarrow X$ and the functor $\mathrm{F}^{1 / r}$ of $r$ th roots of $\mathrm{F}$, a line bundle on $\mathrm{C}$ whose relative degree is a multiple of $r$. We show that it is a $\boldsymbol{\mu}_{r}$-gerbe over a scheme étale on $X$. We notice that the stack of $r$ th roots $\mathrm{F}^{1 / r}$ is finite as soon as the geometric fibre on $X$ is constant.

2. In Section 3.2 , we study the geometric fibres of $\mathrm{F}^{1 / r} \rightarrow X$ by calculating the cohomology of the Kummer sequence

$$
1 \rightarrow \boldsymbol{\mu}_{r} \rightarrow \mathbb{G}_{m} \stackrel{r}{\longrightarrow} \mathbb{G}_{m} \rightarrow 1
$$

for a twisted curve $\mathrm{C}$ over an algebraically closed field. The main result is Theorem 3.2.3, where the endomorphism $\mathrm{L} \mapsto \mathrm{L}^{\otimes r}$ of Pic $\mathrm{C}$ is inscribed in a diagram of exact sequences. In Corollary 3.2.16, we apply the result to twisted curves (we draw the diagram for a twisted curve in Figure 3.2 at 3.2.22). Finally, in Theorem 3.2.2, we deduce the numerical criterion classifying line bundles on twisted curve having exactly $r^{2 g}$ roots. Let us record straight away a consequence of Theorem 3.2 .3 in the context where $r$ divides \#(Aut(e)) for every node e.

3.0.8 Corollary. Let $\pi: \mathrm{C} \rightarrow|\mathrm{C}|$ be a twisted curve of genus $g$ over an algebraically closed field. There is an exact sequence

$$
1 \rightarrow \mathrm{Pic}|\mathrm{C}| \stackrel{\pi^{*}}{\rightarrow} \operatorname{Pic} \mathrm{C} \rightarrow \prod_{\mathrm{e} \in E} \operatorname{Pic} \mathrm{B}(\operatorname{Aut}(\mathrm{e})) \rightarrow 1
$$

where $E$ is the set of nodes e in C. Furthermore, as soon as

$$
\#(\operatorname{Aut}(\mathrm{e})) \in r \mathbb{Z} \quad \forall \mathrm{e} \in E
$$

we can write $\prod_{E} \operatorname{Pic} \mathrm{B}(\operatorname{Aut}(\mathrm{e}))_{r} \cong \mathcal{C}_{1}(\Lambda, \mathbb{Z} / r \mathbb{Z})$ where $\Lambda$ denotes the dual graph of $\mathrm{C}$ and we have an exact sequence

$$
1 \rightarrow(\mathrm{Pic}|\mathrm{C}|)_{r} \stackrel{\pi^{*}}{\rightarrow}(\mathrm{Pic} \mathrm{C})_{r} \rightarrow \mathrm{C}_{1}(\Lambda, \mathbb{Z} / r \mathbb{Z}) \stackrel{\partial}{\rightarrow} \mathrm{C}_{0}(\Lambda, \mathbb{Z} / r \mathbb{Z}) \stackrel{\varepsilon}{\rightarrow} \mathbb{Z} / r \mathbb{Z} \rightarrow 1
$$

where $(\mathrm{Pic}|\mathrm{C}|)_{r}$ and $(\mathrm{Pic} \mathrm{C})_{r}$ denote the $r$-torsion subgroups of the Picard groups, $\partial$ is the boundary homomorphism with respect to a chosen orientation of $\Lambda$, and $\varepsilon$ denotes the augmentation homomorphism sending $\left(h_{v}\right)_{V}$ to $\sum_{V} h_{v} \in \mathbb{Z} / r \mathbb{Z}$.

As a consequence, for any line bundle on $|\mathrm{C}|$, whose total degree is a multiple of $r$, the pullback on $\mathrm{C}$ has $r^{2 g}$ rth roots. (For $(2 g-2) k \in r \mathbb{Z}$, this applies in particular to $\mathrm{F}=\omega_{\mathrm{C}}^{\otimes k}$ by Proposition 2.5.1.) 


\subsection{The stack of $r$ th roots of a line bundle}

Consider the twisted curve $\mathrm{f}: \mathrm{C} \rightarrow X$. We write $\mathrm{LB}_{\mathrm{f}}$ for the category of line bundles on base changes $\mathrm{C}_{S}=\mathrm{C} \times_{X} S$ for every $X$-scheme $S$. More precisely, the objects are pairs $(S, \mathrm{M})$, where $S$ is an $X$-scheme and $\mathrm{M}$ is a line bundle on $\mathrm{C}_{S}=\mathrm{C} \times_{X} S$. The morphisms $(S, \mathrm{M}) \rightarrow\left(S^{\prime}, \mathrm{M}^{\prime}\right)$ are pairs $(m, \mathrm{a})$, where $m \in \operatorname{Hom}_{X}\left(S, S^{\prime}\right)$ and a is an isomorphism of line bundles a: $\mathrm{M} \stackrel{\sim}{\rightarrow} \mathrm{M}^{\prime} \times_{S^{\prime}} S$ on $C_{S}$. This fibred category is an algebraic stack and indeed a substack of the fibred category of coherent sheaves, [Li06, Thm. 2.1.1, Lem. 2.3.1]. The properties of LB $_{f}$ needed here are independently proven in Appendix A (Proposition A.1.2 and Remark A.1.5).

3.1.1 Remark. Note that, in this way, we can define the algebraic stack $\widetilde{\mathrm{LB}}_{g}$ : the category fibred on the category of twisted curves $\widetilde{\mathrm{M}}_{g}$ whose fibre on $\mathrm{C} \rightarrow X$ is the stack $\mathrm{LB}_{\mathrm{f}}$.

Let $\mathrm{F}$ be a line bundle on $\mathrm{C}$, whose relative degree is a multiple of $r$; over $X$ we have $\mathrm{F} \rightarrow \mathrm{C}$. Consider the category $\mathrm{F}^{1 / r}$ of $r$ th roots of $\mathrm{F}_{S}=\mathrm{F} \times_{X} S$ on $\mathrm{C}_{S}=\mathrm{C} \times_{X} S$ for an $X$-scheme $S$. More precisely, the category is formed by the objects $(S, \mathrm{M}, \mathrm{j})$, where $S$ is an $X$-scheme, $\mathrm{M}$ is a line bundle on $\mathrm{C}_{S}$, and $\mathrm{j}$ is an isomorphism $\mathrm{M}^{\otimes r} \stackrel{\sim}{\rightarrow} \mathrm{F}_{S}$. The morphisms $(S, \mathrm{M}, \mathrm{j}) \rightarrow\left(S^{\prime}, \mathrm{M}^{\prime}, \mathrm{j}^{\prime}\right)$ are pairs $(m, a)$ as above, with $a^{\otimes r}$ commuting with $\mathbf{j}$ and $\mathrm{j}^{\prime}$.

3.1.2 Remark. The line bundle $\mathrm{F}$ can be regarded as a section from $X$ to $\mathrm{LB}_{\mathrm{f}}$. Consider the $X$-morphism $\mathrm{k}_{r}: \mathrm{LB}_{\mathrm{f}} \rightarrow \mathrm{LB}_{\mathrm{f}}$ defined by $(S, \mathrm{M}) \mapsto\left(S, \mathrm{M}^{\otimes r}\right)$ and $(m, \mathrm{a}) \mapsto\left(m, \mathrm{a}^{\otimes r}\right)$. In this way, we can equivalently define $\mathrm{F}^{1 / r}$ as the fibre product

$$
\mathrm{F}^{1 / r}=\left(\mathrm{LB}_{\mathrm{f}}\right)_{\mathrm{k}_{r}} \times_{\mathrm{F}} X
$$

3.1.3 Proposition. The category $\mathrm{F}^{1 / r}$ satisfies the following properties.

I. It is a Deligne-Mumford stack, étale and separated over $X$.

II. If $\mathrm{F}=\mathcal{O}$, it is a group stack on $X$.

III. For any $\mathrm{F}$ the functor

$$
\begin{aligned}
\mathrm{m}: \mathcal{O}^{1 / r} \times{ }_{X} \mathrm{~F}^{1 / r} & \rightarrow \mathrm{F}^{1 / r} \\
(\mathrm{~N}, \mathrm{j}),(\mathrm{M}, \mathrm{k}) & \mapsto(\mathrm{N} \otimes \mathrm{M}, \mathrm{j} \otimes \mathrm{k})
\end{aligned}
$$

is an action of $\mathcal{O}^{1 / r}$ on $\mathrm{F}^{1 / r}$, and

$$
\left(\mathrm{m} \times \mathrm{pr}_{2}\right): \mathcal{O}^{1 / r} \times_{X} \mathrm{~F}^{1 / r} \rightarrow \mathrm{F}^{1 / r} \times_{X} \mathrm{~F}^{1 / r}
$$

is an isomorphism of stacks.

Proof. Using [Li06] or Appendix A, the proof of the fact that $\mathrm{F}^{1 / r}$ is of Deligne-Mumford type only amounts to showing that the diagonal is unramified (the category of algebraic stacks is closed under fibred products [LM00, 4.5]). We show that the fibre of the morphism $\operatorname{Isom}_{S}(\alpha, \beta) \rightarrow$ $S$ is reduced. Indeed, at each point $s$ : Spec $k \rightarrow S$ the fibre is either empty, if $\mathrm{M}_{\alpha, s} ¥ \mathrm{M}_{\beta, s}$, or represented by the reduced group scheme $\boldsymbol{\mu}_{r}(k)$, if $\mathbf{M}_{\alpha, s} \cong \mathbf{M}_{\beta, s}$ (the group scheme $\boldsymbol{\mu}_{r}(k)$ acts transitively and freely by multiplication along the fibres of the line bundle).

The étaleness of $\mathrm{F}^{1 / r} \rightarrow X$ claimed in part (I) follows from the fact that the relative cotangent complex vanishes. Indeed, as shown in [ACV03, Prop. 3.0.2] and [AJ03, §2.1], this is a consequence of the fact that the relative cotangent complex $\mathbb{L}_{\mathbf{k}_{r}}$ vanishes. 
In order to show that $\mathrm{F}^{1 / r} \rightarrow X$ is separated it suffices to consider the case $\mathrm{F}=\mathcal{O}$ and to apply the valuative criterion. Let $\left(\operatorname{Spec} R, \mathrm{M}_{R}, \mathrm{j}_{R}\right)$ be an $r$ th root of $\mathcal{O}$ on a twisted curve $\mathrm{C}_{R}$ over a discrete valuation ring $R$. Over the field of fractions $K$, we assume that ( $\operatorname{Spec} K, \mathrm{M}_{R} \otimes K, \mathrm{j}_{R} \otimes K$ ) is trivial: i.e. there exists a trivialization $\mathrm{b}_{K}: \mathrm{M}_{R} \otimes K \rightarrow \mathcal{O}$ compatible with $\mathrm{j}_{R} \otimes K$. Then, the trivialization $\mathrm{b}_{K}$ can be extended over $R$. If we assume that $\mathrm{M}_{R}$ is a pullback from $\left|\mathrm{C}_{R}\right|$, then we can focus on $\left|C_{R}\right| \rightarrow$ Spec $R$ and the claim follows from the separateness of the functor of line bundles of degree zero on each irreducible components of each fibre, Ra70. We claim that $\mathrm{M}_{R}$ is a pullback from $\left|C_{R}\right|$, because the stabilizers at the nodes act trivially on the fibres. This is immediate for nodes that belong to the closure of the nodes of the generic fibre. Otherwise, we can focus on a 'new' node, whose local picture is $\left[\{z y=t\} / \boldsymbol{\mu}_{l}\right]$ for $t \in(\pi) \subset R$. Note that, here, as soon as $\boldsymbol{\mu}_{l}$ acts nontrivially on the fibres, the generic $r$ th root of $\mathcal{O}$ is nontrivial (it induces a nontrivial cyclic covering of the generic fibre).

Part (II) follows from the fact that the stack $\mathcal{O}^{1 / r}$ is a functor sending each $X$-scheme $S$ to the groupoid of $r$-torsion line bundles on $C_{S}$. In fact such a groupoid is a Picard category: a symmetric monoid where each object is invertible and the functors

$$
\begin{aligned}
\mathcal{O}^{1 / r} \times_{X} \mathcal{O}^{1 / r} & \rightarrow \mathcal{O}^{1 / r} \\
\left(S,\left(\mathrm{M}_{1}, \mathrm{j}_{1}\right),\left(\mathrm{M}_{2}, \mathrm{j}_{2}\right)\right) & \mapsto\left(S, \mathrm{M}_{1} \otimes \mathrm{M}_{2}, \mathrm{j}_{1} \otimes \mathrm{j}_{2}\right), \\
X & \rightarrow \mathcal{O}^{1 / r} \\
S & \mapsto(S, \mathcal{O}, \mathrm{id})
\end{aligned}
$$

and

$$
\begin{aligned}
\mathcal{O}^{1 / r} & \rightarrow \mathcal{O}^{1 / r} \\
(S, \mathrm{M}, \mathrm{j}) & \mapsto\left(S, \mathrm{M}^{\vee},\left(\mathrm{j}^{\vee}\right)^{-1}\right)
\end{aligned}
$$

satisfy the law of associativity, the law of the identity, and the law of the inverse.

Part (III) holds because the inverse of the morphism $\mathrm{m} \times \mathrm{pr}_{2}$ is given by the functor $\left(S,\left(\mathrm{M}_{1}, \mathrm{k}_{1}\right),\left(\mathrm{M}_{2}, \mathrm{k}_{2}\right)\right) \mapsto\left(S,\left(\mathrm{M}_{1} \otimes \mathrm{M}_{2}^{\vee}, \mathrm{k}_{1} \otimes\left(\mathrm{k}_{2}^{\vee}\right)^{-1}\right),\left(\mathrm{M}_{2}, \mathrm{k}_{2}\right)\right)$.

3.1.4 Definition (the stack $\overline{\mathrm{F}^{1 / r}}$ ). Note that each object $\left(S, \mathrm{M}, \mathrm{j}\right.$ ) of $\mathrm{F}^{1 / r}$ over an $X$-scheme $S$ has automorphisms given by multiplication by an $r$ th root of unity along the fibre of $\mathrm{L}$. We can eliminate these automorphism by passing to the corresponding rigidified stack (the general setting is recalled in $\S \mathrm{A} .2)$. The stack $\overline{\mathrm{F}^{1 / r}}$ is the rigidification of $\mathrm{F}^{1 / r}$ along $\boldsymbol{\mu}_{r}$ in the sense of [ACV03] (see Theorem A.2.1):

$$
\overline{\mathrm{F}^{1 / r}}=\left(\mathrm{F}^{1 / r}\right)^{\boldsymbol{\mu}_{r}} .
$$

3.1.5 Proposition. The morphism $\overline{\mathrm{F}^{1 / r}} \rightarrow X$ is represented by an étale and separated $X$ scheme, whose geometric fibre on $x$ in $X$ is

$$
\left\{\alpha \in \operatorname{Pic}\left(\mathrm{C}_{x}\right) \mid \alpha^{r}=\left[\mathrm{F}_{x}\right]\right\} .
$$

Assume that $\mathrm{F}$ is the trivial line bundle $\mathcal{O}$ of $\mathrm{C}$; then, $\overline{\mathcal{O}^{1 / r}}$ is a group scheme. 
Proof. The morphism $\mathrm{F}^{1 / r} \rightarrow X$ is étale and separated, Proposition 3.1.3. The morphism $\mathrm{F}^{1 / r} \rightarrow \overline{\mathrm{F}^{1 / r}}$, is surjective and indeed a morphism "locally isomorphic" to $\mathrm{B} \boldsymbol{\mu}_{r}$ on $\overline{\mathrm{F}^{1 / r}}$ (see [Ro, I. Prop. 3.0.2, (2)] and [ACV03, Thm. 5.1.5]). Therefore, $\overline{\mathrm{F}^{1 / r}} \rightarrow X$ is étale and separated.

Finally $\overline{\mathrm{F}^{1 / r}} \rightarrow X$ is representable because for any algebraically closed field $k$ and any $\tau \in \overline{\mathrm{F}^{1 / r}}(\mathrm{Spec} k)$ the automorphism group of $\tau$ is trivial (this condition suffices by a criterion due to Abramovich and Vistoli, AV02, Lem. 4.4.3]). Indeed, the objects of $\overline{F^{1 / r}}=\left(F^{1 / r}\right)^{\mu_{r}}$ over an algebraically closed field $k$ are the same as the objects of $\mathrm{F}^{1 / r}$ over $k$. Now, the automorphism group of the $\boldsymbol{\mu}_{r}$-rigidified object $\tau$ is the quotient by $\boldsymbol{\mu}_{r}(k)$ of the automorphism group of $\tau$ in $\mathrm{F}^{1 / r}$, Theorem A.2.1, (3). This quotient can be easily seen to be trivial: note that $\boldsymbol{\mu}_{r}(k) \rightarrow \operatorname{Aut}(\tau)$ is surjective, because an automorphism of an $r$ th $\operatorname{root}\left(\mathrm{M}, \mathrm{j}: \mathrm{M}^{\otimes r} \stackrel{\sim}{\rightarrow} \mathrm{F}\right)$ on a twisted curve $\mathrm{C} \rightarrow \operatorname{Spec} k$

$$
\mathrm{a}: \mathrm{M} \stackrel{\sim}{\rightarrow} \mathrm{M} \quad \mathrm{a}^{\otimes r} \circ \mathrm{j}=\mathrm{j}
$$

necessarily satisfies $\mathrm{a}^{\otimes r}=\mathrm{id}$ and, since $\mathrm{C} \otimes k$ is connected, a is given by the multiplication by an $r$ th root of unity along the fibre of $\mathrm{M}$.

The rest of the proposition follows immediately from Proposition 3.1 .3 (the group structure and the action of $\mathcal{O}^{1 / r}$ on $\mathrm{F}^{1 / r}$ descend to $\overline{\mathrm{F}^{1 / r}}$ and their relations are preserved).

3.1.6 Proposition. Assume that $\mathrm{C} \rightarrow X$ is a twisted curve and $\mathrm{F} \rightarrow \mathrm{C}$ is a line bundle satisfying the following conditions: for any geometric point $x$ in $X$ the line bundle $\mathrm{F}_{x}$ has $r^{2 g}$ rth roots on $\mathrm{C}_{x}$ up to isomorphism. Then, we have the following properties.

(1) $\overline{\mathcal{O}^{1 / r}}$ is a finite group scheme and $\overline{\mathrm{F}^{1 / r}}$ is a finite torsor under $\overline{\mathcal{O}^{1 / r}}$.

$\left(1^{\prime}\right) \mathcal{O}^{1 / r}$ is a finite group stack and $\mathrm{F}^{1 / r}$ is a finite torsor under $\mathcal{O}^{1 / r}$.

Proof. The morphism $p: Y=\overline{\mathrm{F}^{1 / r}} \rightarrow X$ is étale and the geometric fibres are reduced and consist of $n=r^{2 g}$ distinct points. This condition is sufficient for the properness of $p$ : indeed it implies that $p$ is an isomorphism when $n$ equals 1 , and it implies properness by induction on the degree (we can check properness after an étale base change, and we note that $Y \times_{X} Y$ is finite on $Y$ because $\left(Y \times_{X} Y\right)$ minus the diagonal $\Delta(X)$ is étale and separated on $Y$, and has constant reduced fibre consisting of $n-1$ points).

Now, note that if $\mathrm{F}_{x}$ has $r^{2 g}$ distinct $r$ th roots on $\mathrm{C}_{x}$, then the structure sheaf of $\mathrm{C}_{x}$ also has $r^{2 g}$ distinct roots. Therefore, $\overline{\mathcal{O}^{1 / r}}$ is a finite group and $\overline{\mathrm{F}^{1 / r}}$ is a finite torsor under $\overline{\mathcal{O}^{1 / r}}$, because the geometric fibres of $\overline{\mathrm{F}^{1 / r}} \rightarrow X$ are nonempty, and $\overline{\mathcal{O}^{1 / r}} \times_{X} \overline{\mathrm{F}^{1 / r}} \rightarrow \overline{\mathrm{F}^{1 / r}} \times_{X} \overline{\mathrm{F}^{1 / r}}$ is an isomorphism. Finally the point $\left(1^{\prime}\right)$ follows from Proposition 3.1.3, and the fact that $\mathrm{F}^{1 / r}$ is proper on $\overline{\mathrm{F}^{1 / r}}$.

\subsection{The Kummer theory of a twisted curve}

Since, by the above proposition, $\mathrm{F}^{1 / r}$ is finite as soon as the fibre is constant, we focus on the geometric fibres of $\overline{\mathrm{F}^{1 / r}} \rightarrow X$.

3.2.1 Notation (multiplicity of a line bundle at a smooth stack-theoretic point). We consider 1-dimensional Deligne-Mumford stacks $X$ with nodal singularities and trivial stabilizers except for a finite number of points. As noted in Remark 2.2.8 for each smooth point $\mathrm{p}$ there is a canonical embedding

$$
\mathrm{j}: \mathrm{B}(\operatorname{Aut}(\mathrm{p})) \rightarrow \mathrm{C}
$$


and a canonical generator of $\operatorname{Pic} B(\operatorname{Aut}(p))$ induced by the Aut(p)-linearized tangent space $\mathrm{T}$. With such a canonical generator, the group $\operatorname{Pic} B(\operatorname{Aut}(p))$ can be regarded as $\mathbb{Z} / \#(\operatorname{Aut}(p)) \mathbb{Z}$. Then for any line bundle $F$ at any smooth point $p$ of $X$ we define a multiplicity index

$$
0 \leq \operatorname{mult}_{\mathrm{p}} \mathrm{F} \leq \#(\operatorname{Aut}(\mathrm{p}))-1
$$

via the pullback homomorphism

$$
\operatorname{Pic} X \stackrel{j^{*}}{\rightarrow} \operatorname{Pic} B(\operatorname{Aut}(p))
$$

If $e$ is a node the notation mult $F$ does not make sense, because there is no canonical generator for $\operatorname{Pic} B(\operatorname{Aut}(p))$. Instead, we will consider the pullback of $F$ on the normalization, which is smooth, 1-dimensional, and contains two distinct geometric points $p_{1}$ and $p_{2}$ lying over the node e. By a slight abuse of notation we shall write mult $p_{1} F$ and mult $_{p_{2}} F$ for the multiplicity index at $\mathrm{p}_{1}$ and $\mathrm{p}_{2}$ of the pullback of $\mathrm{F}$ on the normalization.

3.2.2 Theorem. Let $\mathrm{C} \rightarrow$ Spec $k$ be a twisted curve with stabilizers of order $l(\mathrm{e})$ on each node e. Let $\mathrm{F}$ be a line bundle on $\mathrm{C}$ whose total degree is a multiple of $r$.

The number of rth roots of $\mathrm{F}$ is $r^{2 g}$ if and only if the two following numerical conditions are satisfied. First, for each nonseparating node e, $r$ divides

$$
l(\mathrm{e}), m_{1}(\mathrm{e}) \text {, and } m_{2}(\mathrm{e})
$$

where the normalization $\mathrm{C}$ at $\mathrm{e}$ is the connected stack $\mathrm{C}_{0}, \mathrm{p}_{1}, \mathrm{p}_{2} \in \mathrm{C}_{0}$ map to e, and $m_{i}(\mathrm{e})$ equals mult $_{\mathrm{p}_{i}}(\mathrm{~F})$. Second, for each separating node e, $r$ divides

$$
d_{1}(\mathrm{e}) l(\mathrm{e}) \text { and } d_{2}(\mathrm{e}) l(\mathrm{e})
$$

where the normalization of $\mathrm{C}$ at $\mathrm{e}$ is $\mathrm{C}_{1} \sqcup \mathrm{C}_{2}$ and $d_{i} \in(1 / l(\mathrm{e})) \mathbb{Z}$ is the total degree of $\mathrm{F}$ on the curve $\mathrm{C}_{i}$.

In particular, for any line bundle on $|\mathrm{C}|$, whose total degree is a multiple of $r$, there are exactly $r^{2 g}$ roots of the pullback on $\mathrm{C}$ if and only if $r$ divides $l(\mathrm{e})$ for each nonseparating node, and $r$ divides $l(\mathrm{e}) d_{i}(\mathrm{e})$ for each separating node.

We prove the above criterion at the end of the section (3.2.22). The main tool is the exactness of the following diagram (Figure 3.1) and its interpretation for twisted curves (3.2.22, Figure $3.2)$.

3.2.3 Theorem. Let $\pi: \mathrm{X} \rightarrow|\mathrm{X}|$ be a tame and proper Deligne-Mumford stack of dimension 1 with nodal singularities and assume that $\operatorname{Aut}(\mathrm{p})$ is trivial except for a finite number of points $\mathrm{p}_{i}:$ Spec $k \rightarrow \mathrm{X}$. We write $\Gamma_{i}=\operatorname{Aut}\left(\mathrm{p}_{i}\right)$ and we denote by $\mathrm{j}$ the canonical embedding $\sqcup_{i} \mathrm{~B} \Gamma_{i} \rightarrow \mathrm{X}$.

Then, the direct images $R \pi_{*} \mu_{r}$ and $R \pi_{*} \mathbb{G}_{m}$ are represented by complexes on $|\mathrm{X}|$ fitting in the exact sequences

$$
\begin{gathered}
1 \rightarrow \boldsymbol{\mu}_{r} \rightarrow R \pi_{*} \boldsymbol{\mu}_{r} \rightarrow R \pi_{*} \boldsymbol{\mu}_{r} / \boldsymbol{\mu}_{r} \rightarrow 1, \\
1 \rightarrow \mathbb{G}_{m} \rightarrow R \pi_{*} \mathbb{G}_{m} \rightarrow R \pi_{*} \mathbb{G}_{m} / \mathbb{G}_{m} \rightarrow 1 .
\end{gathered}
$$


The long exact sequence of hypercohomology of (3.2.4) yields

$$
\begin{aligned}
& 1 \rightarrow H^{1}\left(|\mathbf{X}|, \boldsymbol{\mu}_{r}\right) \rightarrow H^{1}\left(\mathbf{X}, \boldsymbol{\mu}_{r}\right) \rightarrow \prod_{i} H^{1}\left(\Gamma_{1}, \boldsymbol{\mu}_{r}\right) \\
& \stackrel{\delta}{\longrightarrow} H^{2}\left(|\mathrm{X}|, \boldsymbol{\mu}_{r}\right) \rightarrow H^{2}\left(\mathbf{X}, \boldsymbol{\mu}_{r}\right) \rightarrow \prod_{i} H^{2}\left(\Gamma_{1}, \boldsymbol{\mu}_{r}\right) \rightarrow 1,
\end{aligned}
$$

and the long exact sequence of hypercohomology of (3.2.5) in degree 1 yields

$$
1 \rightarrow \operatorname{Pic}|\mathrm{X}| \stackrel{\pi^{*}}{\rightarrow} \operatorname{Pic} \mathrm{X} \rightarrow \prod_{i} \operatorname{Pic}\left(\mathrm{B}_{i}\right) \rightarrow 1
$$

Both exact sequences above fit in the following commutative diagram (see the second and the third columns), where all vertical and horizontal sequences are exact

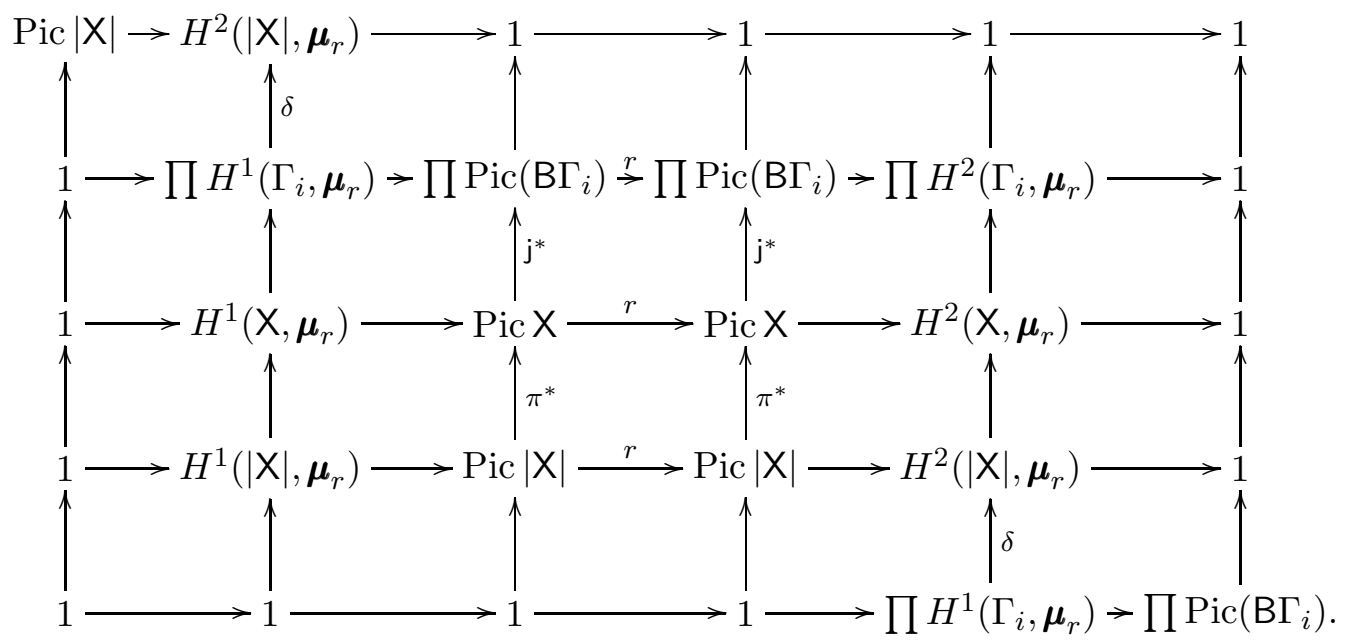

Figure 1: the diagram can be regarded as a double complex $K^{\bullet \bullet \bullet}$ periodic of period $(3,-3)$.

Proof. The exactness of $1 \rightarrow \boldsymbol{\mu}_{r} \rightarrow \mathbb{G}_{m} \rightarrow \mathbb{G}_{m} \rightarrow 1$ holds in the étale topology of $\mathrm{X}$ by the same argument of [Mi80, II.2.18b]. Our goal is to compare the long exact sequences of cohomology of the Kummer sequence of $\mathrm{X}$ and of $|\mathrm{X}|$.

We show that there are three complexes on $|X|$ representing the direct images in the derived category $R \pi_{*} \boldsymbol{\mu}_{r}, R \pi_{*} \mathbb{G}_{m}$, and $R \pi_{*} \mathbb{G}_{m}$ and fitting in a short exact sequence as follows

$$
1 \rightarrow R \pi_{*} \boldsymbol{\mu}_{r} \rightarrow R \pi_{*} \mathbb{G}_{m} \rightarrow R \pi_{*} \mathbb{G}_{m} \rightarrow 1
$$

Indeed, in the category of sheaves of abelian groups on $|\mathrm{X}|$, we can consider three injective resolutions

$$
\begin{aligned}
\boldsymbol{\mu}_{r} & \rightarrow I_{1}^{\bullet}, \\
\mathbb{G}_{m} & \rightarrow I_{2}^{\bullet}, \\
\mathbb{G}_{m} & \rightarrow I_{3}^{\bullet},
\end{aligned}
$$


fitting in

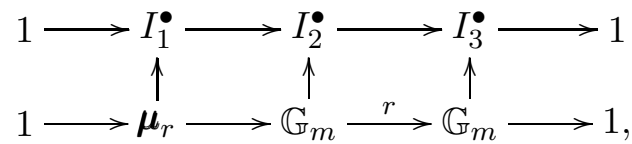

where all the horizontal sequences are exact (note that this happens because the category of sheaves of abelian groups has enough injectives, and, given the injective resolutions $I_{1}^{\bullet}$ and $I_{3}^{\bullet}$, we can also find a third injective resolution $I_{2}^{\bullet}$ making all horizontal sequences exact). Now, applying the direct image via $\pi: \mathrm{X} \rightarrow|\mathrm{X}|$ to $1 \rightarrow I_{1}^{\bullet} \rightarrow I_{2}^{\bullet} \rightarrow I_{3}^{\bullet} \rightarrow 1$, we get the short exact sequence (3.2.6), because all the sheaves involved are injective.

The sequence (3.2.6) allows the comparison of the long exact cohomology sequences attached to the Kummer sequences of $X$ and of $|X|$. Indeed, we show that the Kummer sequence of $|\mathrm{X}|$ injects in (3.2.6). We have $\pi_{*} \boldsymbol{\mu}_{r}=\boldsymbol{\mu}_{r}$ and $\pi_{*} \mathbb{G}_{m}=\mathbb{G}_{m}$, because $\pi_{*} \mathcal{O}_{\mathrm{C}}$ is canonically isomorphic to $\mathcal{O}_{|\mathrm{C}|}$ by Keel and Mori's theorem [KM97], and the isomorphism yields the identity $\pi_{*} \boldsymbol{\mu}_{r}=\boldsymbol{\mu}_{r}$ because it identifies the $r$ th roots of unity. We deduce that, on $|\mathbf{X}|$, the sequence $1 \rightarrow \boldsymbol{\mu}_{r} \rightarrow \mathbb{G}_{m} \rightarrow \mathbb{G}_{m} \rightarrow 1$ injects into (3.2.6). Therefore, we write

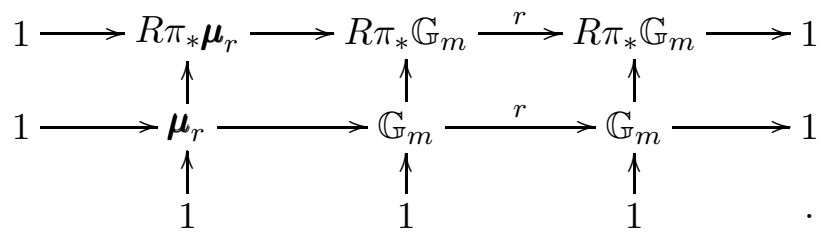

Note that, each vertical sequence in the diagram above is of the form $\pi_{*} \mathrm{~J} \rightarrow R \pi_{*} \mathrm{~J}$, where, on the one hand, $\pi_{*} \mathrm{~J}$ is concentrated in degree $0:\left(1 \rightarrow A^{0} \rightarrow 1 \rightarrow 1 \rightarrow \ldots\right)$, on the other hand $R \pi_{*} \mathrm{~J}$ is $\left(1 \rightarrow B^{0} \rightarrow B^{1} \rightarrow B^{2} \ldots\right)$ and the morphism of complexes is given by

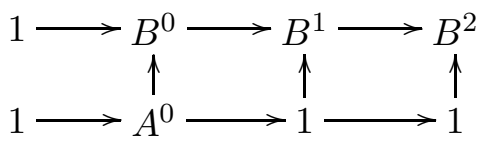

where $A^{0} \rightarrow B^{0}$ is injective. By taking quotients, we get complexes representing $R \pi_{*} \mathrm{~J} / \pi_{*} \mathrm{~J}$ for $\mathrm{J}=\boldsymbol{\mu}_{r}$ or $\mathbb{G}_{m}$. By the exactness of the horizontal sequences in (3.2.7), we get the following diagram where horizontal and vertical sequences are exact.

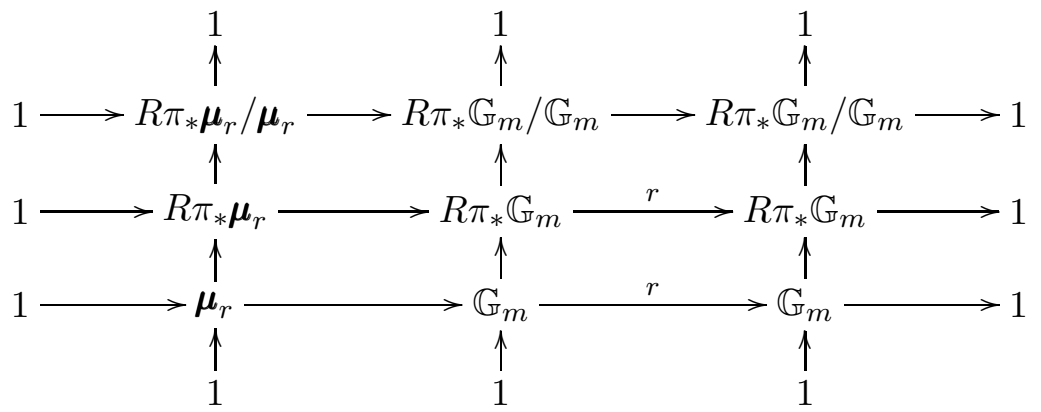

We calculate the hypercohomology groups of $|\mathrm{X}|$ with respect to the complexes $\mathbb{G}_{m}, \boldsymbol{\mu}_{r}$, $R \pi_{*} \mathbb{G}_{m}, R \pi_{*} \boldsymbol{\mu}_{r}, R \pi_{*} \mathbb{G}_{m} / \mathbb{G}_{m}$, and $R \pi_{*} \boldsymbol{\mu}_{r} / \boldsymbol{\mu}_{r}$ in the diagram above. Since the hypercohomology 
groups coincide with the cohomology groups when the complex is concentrated in degree 0 , for any sheaf $E$ on $|\mathrm{X}|$ we have

$$
\mathbb{H}^{j}(|\mathrm{X}|, E)=H^{j}(|\mathrm{X}|, E) .
$$

By the Leray spectral sequence, for any sheaf of groups $\mathrm{J}$ on $\mathrm{X}$, we have

$$
\mathbb{H}^{j}\left(|\mathrm{X}|, R \pi_{*} \mathrm{~J}\right)=H^{j}(\mathrm{X}, \mathrm{J}) \text {. }
$$

Finally the spectral sequence $E_{2}^{p, q}=H^{p}\left(|\mathrm{X}|, H^{q}\left(R \pi_{*} \mathrm{~J} / \pi_{*} \mathrm{~J}\right)\right)$ abuts to $\mathbb{H}^{p+q}\left(|\mathrm{X}|, R \pi_{*} \mathrm{~J} / \pi_{*} \mathrm{~J}\right)$. The sheaf $H^{q}\left(R \pi_{*} \mathrm{~J} / \pi_{*} \mathrm{~J}\right)$ is equal to $R^{q} \pi_{*} \mathrm{~J}$ for $q>0$, and vanishes otherwise. We now notice that $R^{q} \pi_{*} \mathrm{~J}$ is supported on the points with nontrivial stabilizer. Indeed, it is shown in ACV03, Prop. A.0.1] that the stalk of $R^{q} \pi_{*} \mathrm{~J}$ at a point $|\mathrm{p}| \in|\mathrm{X}|$ is canonically isomorphic to the $q$ th cohomology group $H^{q}\left(\operatorname{Aut}(\mathrm{p}), J_{\mathrm{p}}\right)$. Therefore, we have

$$
\mathbb{H}^{j}\left(|\mathbf{X}|, R \pi_{*} \mathrm{~J} / \pi_{*} \mathrm{~J}\right)= \begin{cases}0 & \text { if } j=0 \\ \prod_{i} H^{j}\left(\Gamma_{i}, \mathrm{~J}_{\mathrm{p}_{i}}\right) & \text { if } j>0\end{cases}
$$

because $R^{q} \pi_{*} \mathrm{~J}$ is supported on the points $\left|\mathrm{p}_{i}\right|$, and the differentials of $E_{2}^{p, q}$ vanish.

Passing to the long exact hypercohomology sequences in all directions in the diagram (3.2.8), we get the following diagram. The fact that the diagram (3.2.8) commutes implies that all the squares below commute except for the squares involving the boundary homomorphisms, which anticommute. However, note that all the composition of homomorphism in the squares involving the boundary homomorphisms are trivial, so the following diagram is indeed commutative

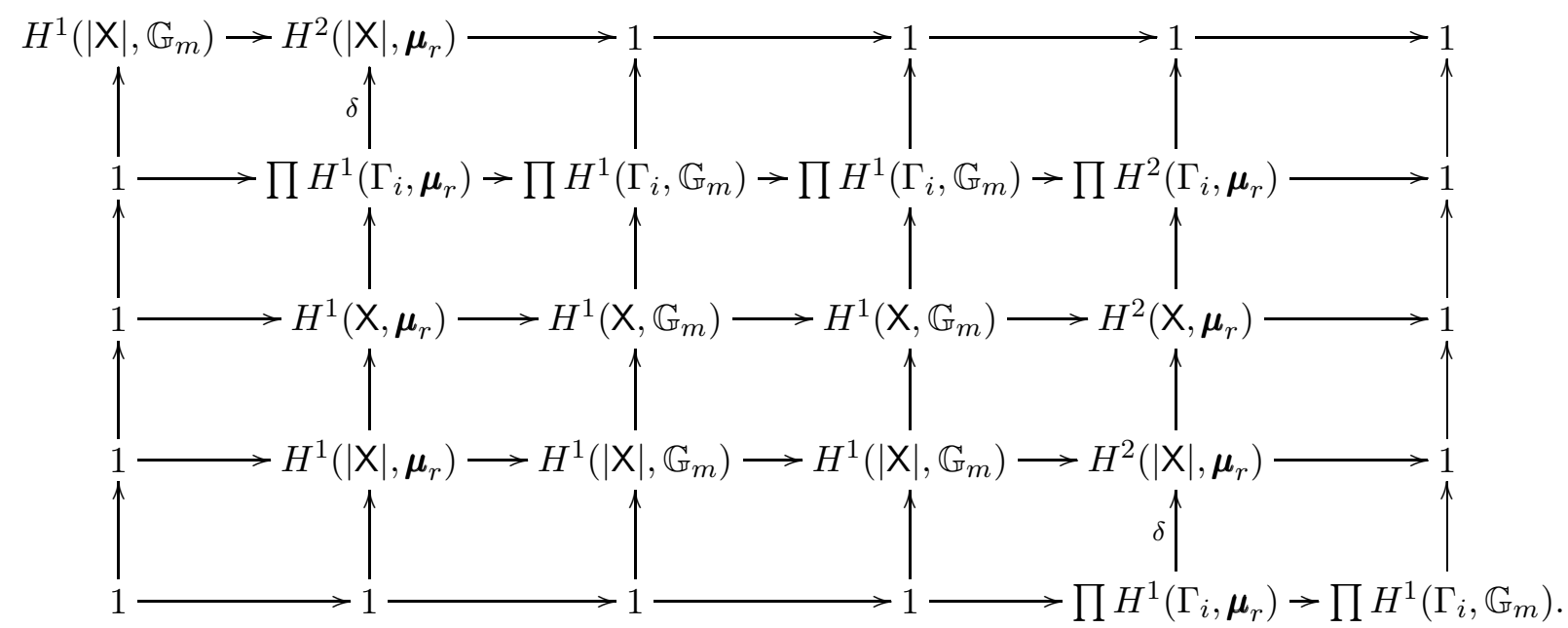

In the diagram above, we used the following facts.

1. For any proper stack $\mathrm{Y}$ the $r$ th power homomorphism is surjective in $H^{0}\left(\mathrm{Y}, \mathbb{G}_{m}\right)$.

2. The coarse space $|X|$ is a 1-dimensional scheme with nodal singularities, therefore we have $H^{2}\left(|\mathrm{X}|, \mathbb{G}_{m}\right)=1$.

3. For $j>0$, the cohomology groups of $\Gamma_{i}=\operatorname{Aut}\left(\mathrm{p}_{i}\right)$ with coefficient in $\left(\mathbb{G}_{m}\right)_{\mathrm{p}_{i}}=\mathbb{G}_{m}$ with a 
trivial $\Gamma_{i}$-action are given by

$$
H^{j}\left(\Gamma_{i}, \mathbb{G}_{m}\right)= \begin{cases}1 & \text { if } j \text { is even, } \\ \mathbb{Z} / l(i) \mathbb{Z} & \text { if } j \text { is odd }\end{cases}
$$

where $l(i)=\#\left(\Gamma_{i}\right)$.

After the identifications of $H^{1}\left(\mathrm{Y}, \mathbb{G}_{m}\right)$ with $\operatorname{Pic}(\mathrm{Y})$, this shows the exactness of the diagram of Figure 3.1.

3.2.12 Remark (the boundary homomorphism $\delta$ ). We show that the coboundary homomorphism

$$
\delta: \prod_{i} H^{1}\left(\Gamma_{i}, \boldsymbol{\mu}_{r}\right) \rightarrow H^{2}\left(|\mathrm{X}|, \boldsymbol{\mu}_{r}\right)
$$

which maps vertically in the commutative diagram of Figure 3.1 can be explicitly computed. When $X$ is a twisted curve, this allows to provide the explicit description of the exact sequence

$$
\begin{aligned}
1 \rightarrow H^{1}\left(|\mathrm{X}|, \boldsymbol{\mu}_{r}\right) \rightarrow H^{1}\left(\mathbf{X}, \boldsymbol{\mu}_{r}\right) \rightarrow & \prod H^{1}\left(\Gamma_{i}, \boldsymbol{\mu}_{r}\right) \\
& \stackrel{\delta}{\rightarrow} H^{2}\left(|\mathrm{X}|, \boldsymbol{\mu}_{r}\right) \rightarrow H^{2}\left(\mathbf{X}, \boldsymbol{\mu}_{2}\right) \rightarrow \prod H^{1}\left(\Gamma_{i}, \boldsymbol{\mu}_{r}\right) \rightarrow 1
\end{aligned}
$$

and to prove Corollary 3.2.16 and the weaker version Corollary 3.0.8 stated above.

First, for any 1-dimensional stack $X$ satisfying the hypotheses of the theorem, we use the double complex of Figure 3.1 to define a $\mathbb{Z} \times \mathbb{Z}$-graded complex $K^{\bullet \bullet \bullet}$ as follows.

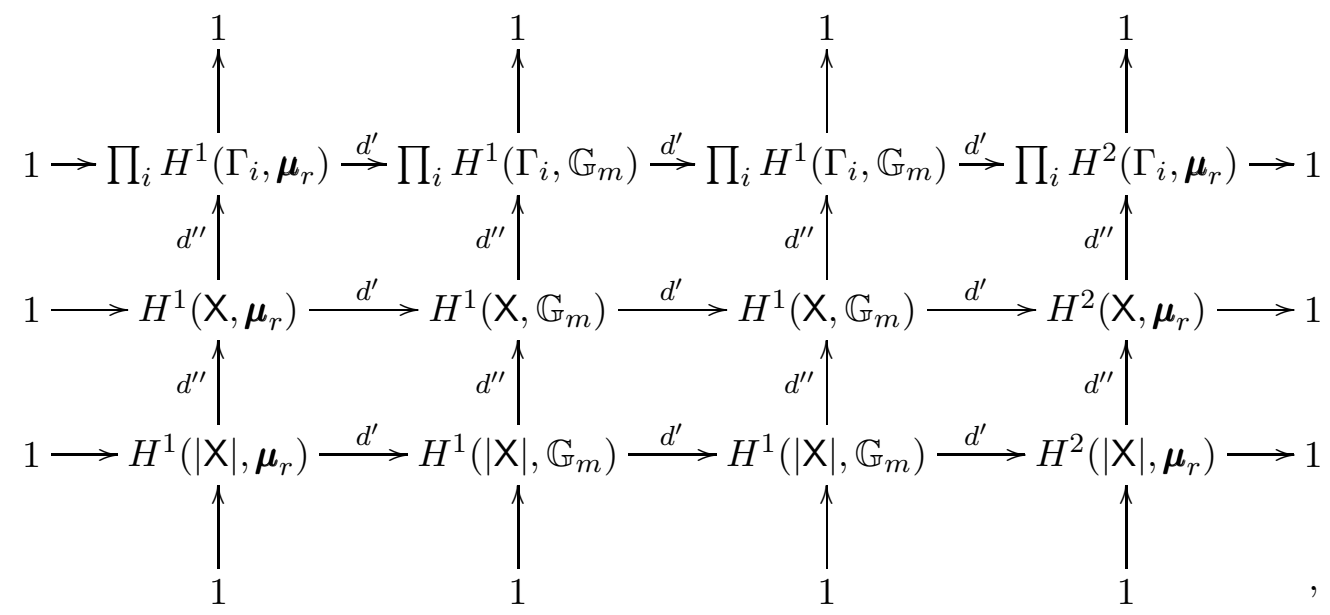

Here $K^{0,0}$ equals $H^{1}\left(|\mathrm{X}|, \boldsymbol{\mu}_{r}\right)$ and all terms $K^{p, q}$ coincide with the terms of the diagram of Figure 3.1 appearing in Theorem 3.2.3 except for $K^{3,-1}=1$ and $K^{0,3}=1$. We also set the differentials $d^{\prime}(p, q): K^{p, q} \rightarrow K^{p+1, q}$ and $d^{\prime \prime}(p, q): K^{p, q} \rightarrow K^{p, q+1}$ as in Figure 3.1, except for $d^{\prime \prime}(0,2)$ and $d^{\prime \prime}(3,-1)$ which are trivial.

Now, by means of the double complex $K^{\bullet} \cdot \bullet$ we identify $\delta$ with a differential ' $d_{3}$ of the spectral sequence associated to the filtration ' $K_{p}=\sum_{i \geq p} K^{i, j}$. This amounts to the following explicit description of $\delta$. For any $x \in \prod_{i} H^{1}\left(\Gamma_{i}, \boldsymbol{\mu}_{r}\right), \delta(x)$ is the (unique) element of $H^{2}\left(|\mathrm{X}|, \boldsymbol{\mu}_{r}\right)$ for 
which there exist $y_{1} \in H^{1}\left(\mathbf{X}, \mathbb{G}_{m}\right)$ and $y_{2} \in H^{1}\left(|\mathbf{X}|, \mathbb{G}_{m}\right)$ satisfying

$$
\left\{\begin{array}{l}
d^{\prime}(x)=d^{\prime \prime}\left(y_{1}\right), \\
d^{\prime}\left(y_{1}\right)=d^{\prime \prime}\left(y_{2}\right), \\
d^{\prime}\left(y_{2}\right)=\delta(x) .
\end{array}\right.
$$

Indeed, we consider the spectral sequences ' $E_{\bullet}$ and ${ }^{\prime \prime} E_{\bullet}$ with

$$
{ }^{\prime} E_{2}^{p, q}=H_{d^{\prime}}^{p}\left(H_{d^{\prime \prime}}^{q}\left(K^{\bullet, \bullet}\right)\right) \quad{ }^{\prime \prime} E_{2}^{p, q}=H_{d^{\prime \prime}}^{q}\left(H_{d^{\prime}}^{p}\left(K^{\bullet, \bullet}\right)\right)
$$

associated to the filtrations ' $K_{p}=\sum_{i \geq p} K^{i, j}$ and ${ }^{\prime \prime} K_{q}=\sum_{j \geq q} K^{i, j}$. Note that $d^{\prime}$ is exact everywhere; therefore, $H_{d^{\prime}}\left(K^{\bullet, \bullet}\right)$ vanishes identically. So, " $E_{2}^{p, q}$ is zero; hence, also ' $E_{h}^{p, q}$ vanishes for all $p$ and $q$ and for $h$ sufficiently large. Since ${ }^{\prime} E_{h}^{p, q}$ is constant for $h \geq 4$, the homomorphism

$$
{ }^{\prime} E_{3}^{0,2}=\operatorname{coker}\left(d^{\prime \prime}(0,1)\right) \stackrel{' d_{3}}{\longrightarrow}{ }^{\prime} E_{3}^{3,0}=\operatorname{ker}\left(d^{\prime \prime}(3,0)\right) .
$$

is an isomorphism. In fact, by construction, the coboundary homomorphism $\delta$ coincides with the composite of $K^{0,2} \rightarrow \operatorname{coker}\left(d^{\prime \prime}(0,1)\right)$ and ' $d_{3}$. The definition of the differential ' $d_{3}$ for the spectral sequence ' $E_{3}^{p, q}$ yields the equations (3.2.14) above.

In the following corollary we apply Theorem 3.2 .3 and the explicit computation of the boundary homomorphism $\delta$ given above to the case of a twisted curve. See also 3.2.22, Figure 3.2 where we summarize the result.

3.2.16 Corollary. Let $\pi: \mathrm{C} \rightarrow|\mathrm{C}|$ be a twisted curve. Write $\Gamma_{\mathrm{e}}$ for Aut(e) for any node e $\in E$ and $l(\mathrm{e})$ for its order. We have a canonical isomorphisms

$$
H^{2}\left(\mathrm{C}, \boldsymbol{\mu}_{r}\right) \cong H^{2}\left(|\mathrm{C}|, \boldsymbol{\mu}_{r}\right) \cong(\mathbb{Z} / r \mathbb{Z})^{V}
$$

Choose an orientation of the dual graph of $\mathrm{C}$; then, we have a canonical isomorphism identifying $H^{1}\left(\Gamma_{\mathrm{e}}, \boldsymbol{\mu}_{r}\right)$ with the $r$-torsion of $\mathbb{Z} / l(\mathrm{e}) \mathbb{Z}$ :

$$
H^{1}\left(\Gamma_{\mathrm{e}}, \boldsymbol{\mu}_{r}\right) \cong(l(\mathrm{e}) / \operatorname{hcf}\{l(\mathrm{e}), r\}) \mathbb{Z} / l(\mathrm{e}) \mathbb{Z} \quad \forall \mathrm{e} .
$$

With respect to these identifications, $\delta: \prod_{\mathrm{e}} H^{1}\left(\Gamma_{\mathrm{e}}, \boldsymbol{\mu}_{r}\right) \rightarrow H^{2}\left(|\mathrm{C}|, \boldsymbol{\mu}_{r}\right)$ in Figure 3.1 as the composite homomorphism of the boundary of the chain complex $\mathcal{C}_{\bullet}(\Lambda, \mathbb{Z} / r \mathbb{Z})$ of the dual graph $\Lambda$ of $\mathrm{C}$ and of the homomorphism $\prod_{\mathrm{e}} H^{1}\left(\Gamma_{\mathrm{e}}, \boldsymbol{\mu}_{r}\right) \rightarrow(\mathbb{Z} / r \mathbb{Z})^{E}$ given by the product over $E$ of

$$
(l(\mathrm{e}) / \operatorname{hcf}\{l(\mathrm{e}), r\}) \mathbb{Z} / l(\mathrm{e}) \mathbb{Z} \stackrel{\times(r / l(\mathrm{e}))}{\longrightarrow}(r / \operatorname{hcf}\{l(\mathrm{e}), r\}) \mathbb{Z} / r \mathbb{Z} \hookrightarrow \mathbb{Z} / r \mathbb{Z} \quad \forall \mathrm{e}
$$

Proof. First, we see the preliminary case of the stack $C[p / l]$. Then, we normalize the twisted curve and use the presentation of smooth stacks of dimension 1 given in Section 2.2.7.

3.2.19 Lemma. Let $C$ be a proper, connected, smooth, and reduced curve. For $p \in C$ and $l$ an invertible integer, consider the stack $C[p / l]$ : the point $C[p / l]$ lying over $p \in C$ has automorphism 
group $\Gamma$ of order $l$. The boundary homomorphism $\delta$ of Figure 3.1 is the composite homomorphism

$$
H^{1}\left(\mathrm{~B} \Gamma, \boldsymbol{\mu}_{r}\right) \cong(l / \operatorname{hcf}\{l, r\}) \mathbb{Z} / l \mathbb{Z} \stackrel{\times(r / l)}{\longrightarrow}(r / \operatorname{hcf}\{l, r\}) \mathbb{Z} / r \mathbb{Z} \hookrightarrow \mathbb{Z} / r \mathbb{Z} \cong H^{2}\left(C, \boldsymbol{\mu}_{r}\right),
$$

where we used the canonical isomorphisms $\operatorname{Pic}(\mathrm{B \Gamma}) \cong \mathbb{Z} / l \mathbb{Z}$ (Remark 2.2.8) and $H^{2}\left(C, \boldsymbol{\mu}_{r}\right) \cong$ $\mathbb{Z} / r \mathbb{Z}$.

Proof. As in Remark 2.2.8, we denote by $\mathrm{T}$ the tangent space at p. Now, we apply to $\mathrm{T}^{\otimes m} \in$ $H^{1}\left(\Gamma, \boldsymbol{\mu}_{r}\right)$ the definition of $\delta$ given in (3.2.14). Let $\mathrm{M}$ be a line bundle on $C[p / l]$ such that $\mathrm{j}^{*} \mathrm{M}=\mathrm{T}^{\otimes m}$; let $A$ be a line bundle on $C$, such that $\pi^{*} A=\mathrm{M}^{\otimes r}$. Then, $\delta\left(\mathbf{T}^{\otimes m}\right)$ is $\operatorname{deg} A \bmod r$. Finally, by Proposition 2.2.10, we have

$$
\operatorname{deg}(A)=\operatorname{deg}\left(\mathbf{M}^{\otimes r}\right)=r \operatorname{deg}(\mathbf{M})=r(k+m / l)=r k+m r / l .
$$

This is indeed the claim of the lemma.

Now let $D \rightarrow C$ and $|D| \rightarrow|C|$ be the normalization of $C$ and $|C|$. Note that $D$ has trivial stabilizers except for a finite set of smooth points $I$. Therefore, Theorem 3.2 .3 applies. Furthermore, for any point $\mathrm{p} \in \mathrm{D}$ with $|\operatorname{Aut}(\mathrm{p})|=l>1$, there is a natural projection

$$
\mathrm{D} \rightarrow|\mathrm{D}|[|\mathrm{p}| / l]
$$

Using Lemma 3.2.19, we get a canonical isomorphism from $H^{1}\left(\operatorname{Aut}(\mathrm{p}), \boldsymbol{\mu}_{r}\right)$ to $(l / \operatorname{hcf}\{l, r\}) \mathbb{Z} / l \mathbb{Z}$ and a homomorphism

$$
H^{1}\left(\operatorname{Aut}(\mathrm{p}), \boldsymbol{\mu}_{r}\right) \stackrel{\times(r / l)}{\longrightarrow} H^{2}\left(|\mathrm{D}|, \boldsymbol{\mu}_{r}\right) .
$$

Note that the morphism $\delta_{\mathrm{D}}: \prod_{\mathrm{p} \in I} H^{1}\left(\operatorname{Aut}(\mathrm{p}), \boldsymbol{\mu}_{r}\right) \rightarrow H^{2}\left(|\mathrm{D}|, \boldsymbol{\mu}_{r}\right)$ coincides with (3.2.21) on each factor, because $\delta$ commutes with pullbacks via the projections (3.2.20).

Now, consider $D \rightarrow C$. For each node e of $C$ there are two smooth points $p_{e,+}, p_{e,-} \in$ D over e with stabilizer $\Gamma_{\mathrm{e}}$, we denote them according to the orientation of the dual graph. Now, $H^{1}\left(\Gamma_{\mathrm{e}}, \boldsymbol{\mu}_{r}\right)$ is the $r$-torsion subgroup of $H^{1}\left(\Gamma_{\mathrm{e}}, \mathbb{G}_{m}\right)$, which is canonically generated by the tangent space $\mathrm{T}_{\mathrm{e},+}$ at the point of $\mathrm{p}_{\mathrm{e},+} \in \mathrm{D}$. Thus, by raising $\mathrm{T}_{\mathrm{e},+}$ to the $(l(\mathrm{e}) / \operatorname{hcf}\{l(\mathrm{e}), r\})$ th power, we get the canonical isomorphism (3.2.18).

The cohomology homomorphisms induced by pullback via $\mathrm{D} \rightarrow \mathrm{C}$ are compatible with the diagrams of Figure 3.1 for C and D. Now,

$$
\delta_{\mathrm{C}}: \prod_{\mathrm{e}} H^{1}\left(\Gamma_{\mathrm{e}}, \boldsymbol{\mu}_{r}\right) \rightarrow H^{2}\left(|\mathrm{C}|, \boldsymbol{\mu}_{r}\right)
$$

fits in the following commutative diagram

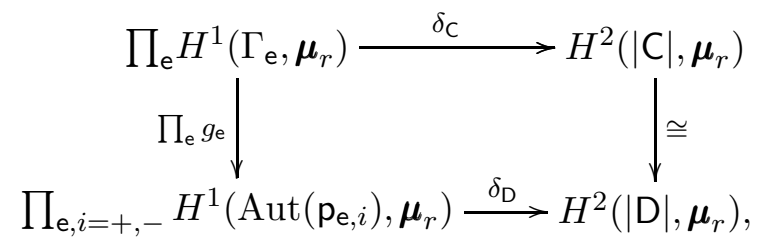


where the homomorphism induced via pullback $H^{2}\left(|\mathrm{D}|, \boldsymbol{\mu}_{r}\right) \rightarrow H^{2}\left(|\mathrm{C}|, \boldsymbol{\mu}_{r}\right)$ is invertible and the morphisms $g_{\mathrm{e}}$ are induced by pullback via $\mathrm{D} \rightarrow \mathrm{C}$ and map as

$$
\mathrm{T}_{\mathrm{e},+} \rightarrow\left(\mathrm{T}_{\mathrm{e},+},\left(\mathrm{T}_{\mathrm{e},+}\right)^{\vee}\right) \quad \forall \mathrm{e}
$$

This happens because, by the definition of twisted curves 2.4.1, the local picture of $\mathrm{C}$ at $\mathrm{e}$ is $\{z w=0\}$ with $\boldsymbol{\mu}_{l(\mathrm{e})}$ acting as $(z, w) \mapsto\left(\xi_{l(\mathrm{e})} z, \xi_{l(\mathrm{e})}^{-1} w\right)$.

3.2.22. Proof of Theorem 3.2.2. We prove the claim by chasing in the following diagram derived from Theorem 3.2 .3 and Corollary 3.2 .16 .

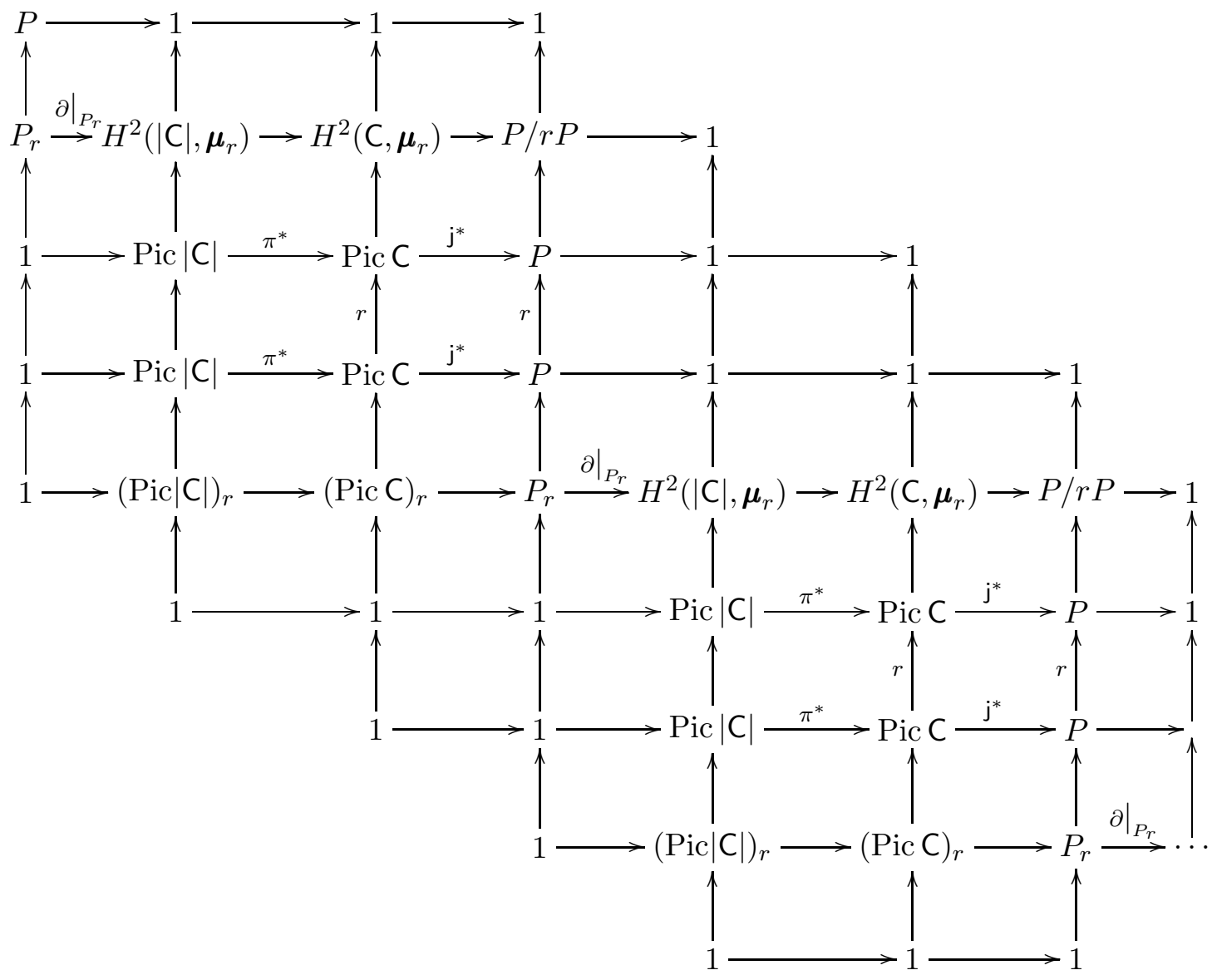

Figure 2: the diagram is commutative and all horizontal and vertical sequences are exact.

In the figure above we used the following notation:

1. $P_{\mathrm{e}}$ denotes $\mathrm{Pic}(\mathrm{B}$ Aut $(\mathrm{e}))$, and $P$ denotes $\prod_{\mathrm{e}} P_{\mathrm{e}}$;

2. for any group $H, H_{r}$ denotes the $r$-torsion subgroup;

3. $\partial$ denotes the differential of $\mathcal{C}_{\bullet}(\Lambda, \mathbb{Z} / r \mathbb{Z})$ for $\Lambda$ the dual graph of $C$ with an orientation; 
4. $\mathrm{j}$ denotes the embedding of the singular locus in $\mathrm{C}$;

5. $\pi$ denotes the morphism from $\mathrm{C}$ to $|\mathrm{C}|$.

We prove Theorem 3.2 .2 in two steps: we first focus on roots of $\mathcal{O}$; then, we consider the roots of $F$.

Step 1. The special case: $r$ th roots of $\mathcal{O}$. We need to show that there are exactly $r^{2 g}$ roots of $\mathcal{O}$ if and only if $l(\mathrm{e})$ is a multiple of $r$ for any nonseparating node e. By Theorem 3.2 .3 (see Figure $3.2)$, the number of elements of $\operatorname{Pic}(\mathrm{C})_{r}$ equals the product of $\#\left((\operatorname{Pic}|\mathrm{C}|)_{r}\right)=r^{2 g-1+\#(V)-\#(E)}$ by the size of the kernel of

$$
\partial: P_{r} \rightarrow H^{2}\left(|\mathrm{C}|, \boldsymbol{\mu}_{r}\right) .
$$

Therefore, it is enough to show the following claim

$$
\#(\operatorname{ker} \partial)=r^{1-\#(V)+\#(E)} \Leftrightarrow l(\mathrm{e}) \in r \mathbb{Z} \quad \forall \mathrm{e} \text { nonseparating. }
$$

Recall that the first Betti number of the dual graph $\Lambda$ of $\mathrm{C}$ is given by $b_{1}(\Lambda)=1-\#(V)+\#(E)$. Consider the subgraph $\Lambda(\mathrm{e})$ of $\Lambda$ of $|\mathrm{C}|$, whose vertices and edges are $V$ and $E \backslash\{\mathrm{e}\}$, denote by $\widehat{\partial}_{\mathrm{e}}$ the restriction of the chain differential of $\Lambda(\mathrm{e})$ to $P_{r}(\mathrm{e})=\prod_{E \backslash\{\mathrm{e}\}}\left(P_{\mathrm{e}}\right)_{r}$. For any nonseparating $\mathrm{e} \in E$, we have

$$
\#(\operatorname{ker} \partial)=\#\left(\operatorname{ker} \widehat{\partial}_{\mathrm{e}}\right) \cdot \operatorname{hcf}\{r, l(\mathrm{e})\}
$$

The claim (3.2.24) follows. Indeed, assume \#(ker $\partial)=r^{1-\#(V)+\#(E)}$. Now, we have $\#\left(\operatorname{ker} \widehat{\partial}_{\mathrm{e}}\right) \leq$ $r^{b_{1}(\Lambda(\mathrm{e}))}$ and $b_{1}(\Lambda(\mathrm{e}))=b_{1}(\Lambda)-1$ if e is nonseparating. Hence, $r=\operatorname{hcf}\{r, l(\mathrm{e})\}$. Conversely, assume $l(\mathrm{e}) \in r \mathbb{Z}$ for all nonseparating e. The claim holds when there are $n-1$ nonseparating edges, then (3.2.25) implies \# $(\operatorname{ker} \partial)=r^{b_{1}(\Lambda(e))+1}=r^{1-\#(V)+\#(E)}$.

Step 2. The general case: rth roots of $\mathrm{F}$. We assume the numerical condition in the statement, and we show that it implies that F has one $r$ th root (by Step 1, this also implies that the number of roots is $r^{2 g}$ ).

By the diagram in Figure 3.2, this amounts to a combinatorial criterion on the differential of $\mathcal{C}^{\bullet}(\Lambda, \mathbb{Z} / r \mathbb{Z})$ (recall that an orientation for $\Lambda$ is chosen). The point is that for any line bundle $A$ on the curve $|\mathrm{C}|$ the pullback $\pi^{*} A$ has an $r$ th root in $\operatorname{Pic}(\mathrm{C})$ if and only if $\operatorname{deg}(A)$ is in the image of $\partial$ in Figure 3.2 .

In order to state the criterion we attach a partition of $E \backslash\{\mathrm{e}\}$ and $V$ to each separating node e joining $v_{+}$and $v_{-}$:

$$
E \backslash\{\mathrm{e}\}=E^{+} \sqcup E^{-} \quad V=V^{+} \sqcup V^{-},
$$

where the set $E^{+}$(the set $V^{+}$) contains the edges (the vertices) that can be connected to $v_{+}$ without passing through e. Then, regard $P_{r}$ as the product $\prod_{\mathrm{e} \in E}\left(P_{\mathrm{e}}\right)_{r}$ and write $P^{ \pm}(\mathrm{e})=$ $\prod_{\mathrm{e} \in E^{ \pm}}\left(P_{\mathrm{e}}\right)_{r}$, write $\partial_{\mathrm{e}}^{ \pm}$for the corresponding chain differentials, and denote by $\varepsilon_{\mathrm{e}}^{ \pm}$the composition of $V^{ \pm} \hookrightarrow V$ with the augmentation homomorphism $\varepsilon: V \rightarrow \mathbb{Z} / r \mathbb{Z}$ (over ker $\varepsilon$, we obviously have $\left.\varepsilon_{\mathrm{e}}^{+}=-\varepsilon_{\mathrm{e}}^{-}\right)$.

3.2.26 Lemma. Assume that $r$ divides $l(\mathrm{e})$ if e is nonseparating. Consider an element $\vec{t} \in$ ker $\varepsilon \in(\mathbb{Z} / r \mathbb{Z})^{V}$; the following conditions are equivalent.

1. $\vec{t}$ is in the image of $P_{r} \subseteq(\mathbb{Z} / r \mathbb{Z})^{E}$ via $\partial$. 
2. For any separating edge e, the value of $\varepsilon_{\mathrm{e}}^{+}(\vec{t}) \in \mathbb{Z} / r \mathbb{Z}$ belongs to $P_{\mathrm{e}}$ (or, equivalently, the value of $\varepsilon_{\mathrm{e}}^{-}(\vec{t})=-\varepsilon_{\mathrm{e}}^{+}(\vec{t})$ belongs to $\left.P_{\mathrm{e}}\right)$.

Proof. Note that the claim is trivial if all edges are nonseparating. Indeed, (2) is true, whereas (11) is true, because if $\varepsilon(\vec{t})$ vanishes, then $\vec{t}$ is exact in $\mathcal{C}^{\bullet}$ and lies in $\partial\left(P_{r}\right)$ by $l(\mathrm{e}) \in r \mathbb{Z}$.

Then, choose a separating edge e joining $v^{+}$to $v^{-}$Note that, the homomorphism $\partial$ can be written as

$$
\begin{array}{rlll}
P^{+}(\mathrm{e}) \times\left(P_{\mathrm{e}}\right)_{r} \times P^{+}(\mathrm{e}) & \rightarrow & & (\mathbb{Z} / r \mathbb{Z})^{V^{+}} \times \quad \times \quad(\mathbb{Z} / r \mathbb{Z})^{V^{-}} \\
(a, x, b) & \mapsto & & \left(\partial_{\mathrm{e}}^{+}(a)+i_{+}(x), \quad \partial_{\mathrm{e}}^{-}(b)-i_{-}(x)\right),
\end{array}
$$

where $i^{+}$and $i^{-}$are the injections of $\left(P_{\mathrm{e}}\right)_{r}$ in $(\mathbb{Z} / r \mathbb{Z})^{V^{+}}$and $(\mathbb{Z} / r \mathbb{Z})^{V^{-}}$induced by $v^{+} \in V^{+}$ and $v^{-} \in V^{-}$.

We assume $\vec{t} \in \partial\left(P_{r}\right)$, and we prove (2). Indeed, there exist $a \in P^{+}(\mathrm{e})$ and $x \in\left(P_{\mathrm{e}}\right)_{r}$ satisfying $\varepsilon_{\mathrm{e}}^{+}(\vec{t})=\varepsilon_{\mathrm{e}}^{+}\left(\partial_{\mathrm{e}}^{+}(a)+i_{+}(x)\right)$. Therefore, we have $\varepsilon_{\mathrm{e}}^{+}(\vec{t})=\varepsilon_{\mathrm{e}}^{+}\left(i_{+}(x)\right)=x \in P_{\mathrm{e}}$.

Conversely, choose a separating edge e, and set $x_{\mathrm{e}}=\varepsilon_{\mathrm{e}}^{+}(\vec{t}) \in\left(P_{\mathrm{e}}\right)_{r}$. We construct $\vec{h} \in P_{r}$ such that $\partial(\vec{h})=\vec{t}$, by means of the presentation of $\partial$ given in the diagram above. Using the partition $V=V^{+} \sqcup V^{-}$, write $\vec{t}$ as $\left(\vec{t}_{\mathrm{e}}^{+}, \vec{t}_{\mathrm{e}}^{-}\right)$. Now, giving $\vec{h}$ is equivalent to finding two elements mapping via $\partial_{\mathrm{e}}^{+}$and $\partial_{\mathrm{e}}^{-}$to $\vec{q}_{\mathrm{e}}^{+}=\vec{t}_{\mathrm{e}}^{+}-i_{\mathrm{e}}^{+}\left(x_{\mathrm{e}}\right)$ and $\vec{q}_{\mathrm{e}}^{-}=\vec{t}_{\mathrm{e}}^{-}+i_{\mathrm{e}}^{+}\left(x_{\mathrm{e}}\right)$. By induction on the number of separating edges, the lemma holds on the subgraphs with edges $E^{+}$(or $E^{-}$) and vertices $V^{+}$ (or $V^{-}$). Therefore, in order to lift $\vec{q}=\vec{q}_{\mathrm{e}}^{+}$and $\vec{q}_{\mathrm{e}}^{-}$to $P^{+}(\mathrm{e})$ and $P^{-}(\mathrm{e})$, we only need to show that $\vec{q}$ satisfies (2). This is immediate, since, by construction, for any separating edge $\mathrm{f}$ we have either $\varepsilon_{\mathrm{f}}^{+}(\vec{q})=\varepsilon_{\mathrm{f}}^{+}(\vec{t})$ or $\varepsilon_{\mathrm{f}}^{-}(\vec{q})=\varepsilon_{\mathrm{f}}^{-}(\vec{t})$.

Assuming that the numerical condition on the nodes is satisfied, we construct an $r$ th root of F. Using the orientation of the dual graph $\Lambda$ chosen above, we have a canonical way to associate to a node e a point $\mathrm{p}_{+}$over e lying in the normalization of $\mathrm{C}$ at e. We set $m(\mathrm{e})=\operatorname{mult}_{\mathrm{p}_{+}}(\mathrm{F})$. In the same way, for any separating node e, the orientation allows us to associate to e a component $\mathrm{C}_{+}(\mathrm{e})$ of the partial normalization of $\mathrm{C}$ at e. Then, we set $d(\mathrm{e})=\operatorname{deg}_{\mathrm{C}_{+}(\mathrm{e})}(\mathrm{F})$. By hypothesis there exists a function $k(\mathrm{e}) \in \mathbb{Z}$ satisfying

$$
\begin{array}{ll}
d(\mathrm{e}) l(\mathrm{e})=k(\mathrm{e}) r & \text { for separating nodes and } \\
m(\mathrm{e})=k(\mathrm{e}) r & \text { for nonseparating nodes. }
\end{array}
$$

The orientation of the dual graph $\Lambda$ also induces a canonical generator $T_{p_{+}}$for each group $\operatorname{Pic}(\operatorname{B~Aut}(\mathrm{e}))$. We consider

$$
\prod_{\mathrm{e}} \mathrm{T}_{\mathrm{p}_{+}}^{\otimes k(\mathrm{e})} \in \prod_{\mathrm{e}} P_{\mathrm{e}}
$$

Let $\mathrm{M}$ be a line bundle on $\mathrm{C}$ such that $\mathrm{j}^{*} \mathrm{M}=\prod_{\mathrm{e}} \mathrm{T}_{\mathrm{p}_{+}}^{\otimes k(\mathrm{e})}$. By the equations (3.2.27) and (3.2.28) and Proposition 2.2.10, we have $\mathrm{j}^{*}\left(\mathrm{M}^{\otimes r}\right)=\mathrm{j}^{*} \mathrm{~F}$. Therefore, there exists a line bundle $A \in \mathrm{Pic}|\mathrm{C}|$ satisfying $\pi^{*} A=\left(\mathrm{M}^{\otimes r}\right)^{\vee} \otimes \mathrm{F}$.

In fact, $\pi^{*} A$ has an $r$ th root, because it lies in the kernel of $\operatorname{Pic}(\mathrm{C}) \rightarrow H^{2}\left(\mathrm{C}, \boldsymbol{\mu}_{r}\right)$. To see this, using the diagram of Figure 3.2, it is enough to show that the homomorphism $\mathrm{Pic}|\mathrm{C}| \rightarrow$ $H^{2}\left(|\mathrm{C}|, \boldsymbol{\mu}_{r}\right)$ sends $A$ into $\partial\left(P_{r}\right)$. We apply Lemma 3.2.26 to the multidegree of $A \bmod r$; clearly, condition (2) of the lemma holds if the following numerical condition is satisfied for each sepa- 
rating node: the total degree of $\pi^{*} A$ on the connected component $\widetilde{\mathrm{C}}=\mathrm{C}_{+}(\mathrm{e})$ is a multiple of $r$. Indeed, $r$ divides

$$
\operatorname{deg}\left(\left.\pi^{*} A\right|_{\widetilde{\mathrm{C}}}\right)=\left.\operatorname{deg}\left(\left(\mathrm{M}^{\otimes r}\right)^{\vee} \otimes \mathrm{F}\right)\right|_{\widetilde{\mathrm{C}}}=-r \operatorname{deg}\left(\left.\mathrm{M}\right|_{\widetilde{\mathrm{C}}}\right)+d(\mathrm{e})=r\left(-\operatorname{deg}\left(\left.\mathrm{M}\right|_{\tilde{\mathrm{C}}}\right)+d(\mathrm{e}) / r\right)
$$

because $\operatorname{deg}\left(\left.\mathrm{M}\right|_{\tilde{\mathrm{C}}}\right)-k(\mathrm{e}) / l(\mathrm{e})$ is an integer by Proposition 2.2 .10 and we have $k(\mathrm{e}) / l(\mathrm{e})=d(\mathrm{e}) / r$ by (3.2.27). This proves the claim, because by tensoring a root of $\pi^{*} A$ by $\mathrm{M}$ we get a root of $\mathrm{F}$.

Conversely, if $\mathrm{F}$ has $r^{2 g} r$ th roots, we show $l(\mathrm{e}), m(\mathrm{e}) \in r \mathbb{Z}$ for any nonseparating node e and $l(\mathrm{e}) d(\mathrm{e}) \in r \mathbb{Z}$ for any separating node e. First, for any nonseparating node e, Step 1 implies $l(\mathrm{e}) \in r \mathbb{Z}$, and, since $\mathrm{F}$ has an $r$ th root, j*F also has an $r$ th root, and we have $m(\mathrm{e}) \in r \mathbb{Z}$. Second, we show $l(\mathrm{e}) d(\mathrm{e}) \in r \mathbb{Z}$ for a separating node e. Since $\mathrm{j}^{*} \mathrm{~F}$ has an $r$ th root and $\mathrm{j}^{*}$ is surjective, we can choose a line bundle $\mathrm{M}$ on $\mathrm{C}$ such that $\mathrm{j}^{*} \mathrm{M}^{\otimes r}=\mathrm{j}^{*} \mathrm{~F}$. There exists $A \in \mathrm{Pic}|\mathrm{C}|$ such that $\pi^{*} A=\left(\mathrm{M}^{\otimes r}\right)^{\vee} \otimes \mathrm{F}$. Since $\mathrm{F}$ has an $r$ th root, $\pi^{*} A$ has an $r$ th root; therefore, the homomorphism Pic $|\mathrm{C}| \rightarrow H^{2}\left(|\mathrm{C}|, \boldsymbol{\mu}_{r}\right)$ sends $A$ into the image of $\partial: P_{r} \rightarrow H^{2}\left(|\mathrm{C}|, \boldsymbol{\mu}_{r}\right)$. Note that, by Lemma 3.2.26, the degree of $\pi^{*} A$ on $\mathrm{C}_{+}(\mathrm{e})$ has order $l(\mathrm{e})$ modulo $r$; hence, we have

$$
l(\mathrm{e}) \operatorname{deg}\left(\left.\pi^{*} A\right|_{\mathrm{C}_{+}(\mathrm{e})}\right) \in r \mathbb{Z},
$$

which implies $l(\mathrm{e}) d(\mathrm{e}) \in r \mathbb{Z}$, because

$$
l(\mathrm{e}) \operatorname{deg}\left(\left.\pi^{*} A\right|_{\mathrm{C}_{+}(\mathrm{e})}\right) \quad=\left.\quad l(\mathrm{e}) \operatorname{deg}\left(\mathrm{j}^{*}\left(\mathrm{M}^{\otimes r}\right)^{\vee} \otimes \mathrm{F}\right)\right|_{\mathrm{C}_{1}}=-r l(\mathrm{e}) \operatorname{deg}\left(\left.\mathrm{M}\right|_{\mathrm{C}_{1}}\right)+l(\mathrm{e}) d(\mathrm{e}) .
$$

\section{The notion of stability for twisted curves}

\subsection{Twisted curves and the notion of $\vec{l}$-stability}

By Olsson's Theorem 2.4.6, the category of twisted curves of genus $g \geq 2$ forms a DeligneMumford stack. As the following example shows, the stack $\widetilde{\mathrm{M}}_{g}$ is not separated.

4.1.1 Example. A twisted curve $\mathrm{C}$ over a discrete valuation ring $R$ with smooth generic fibre $\mathrm{C}_{K}$ is isomorphic to its coarse space over $K$ and may differ from it on the special fibre; in this case the coarse space $|\mathrm{C}|$ and $\mathrm{C}$ are two nonisomorphic twisted curves extending $\mathrm{C}_{K}$ on $S$. Therefore, the valuative criterion of separateness fails.

Inside $\widetilde{\mathrm{M}}_{g}$, we identify all proper substacks containing $\mathrm{M}_{g}$. We need to recall the following standard notion of type of a node.

4.1.2 Notation (type of a node). Given a twisted curve of genus $g \geq 2$ over an algebraically 
closed field $k$ and a node $e \in \mathrm{C}$ we set the following convention:

$$
e \in \mathrm{C} \text { is } \begin{cases}\text { of type } 0 & \text { if the normalization of } \mathrm{C} \text { at e } \\ & \text { is connected (i.e. e is nonseparating); } \\ \text { of type } i \text { with } & \text { if normalizing } \mathrm{C} \text { at e we get } \mathrm{C}_{1} \sqcup \mathrm{C}_{2} \\ 1 \leq i \leq\lfloor g / 2\rfloor & \text { with }\left\{g\left(\mathrm{C}_{1}\right), g\left(\mathrm{C}_{2}\right)\right\}=\{i, g-i\} .\end{cases}
$$

4.1.3 Definition. Let $\vec{l}=\left(l_{0}, l_{1}, \ldots, l_{\lfloor g / 2\rfloor}\right)$ be a multiindex of positive and invertible integers $l_{i}$. A twisted curve $\mathrm{C} \rightarrow X$ is $\vec{l}$-stable, if the coarse space is stable and if the stabilizer at a node of type $i$ has order $l_{i}$.

4.1.4 Theorem. Let us denote by $\mathrm{M}_{g}(\vec{l})$ the category of $\vec{l}$-stable curves. It is contained in $\widetilde{\mathrm{M}}_{g}$ and it contains $\mathrm{M}_{g}$ :

$$
\mathrm{M}_{g} \hookrightarrow \mathrm{M}_{g}(\vec{l}) \hookrightarrow \widetilde{\mathrm{M}}_{g}
$$

I. The stack $\mathrm{M}_{g}(\vec{l})$ is tame, proper (separated), smooth, irreducible and of Deligne-Mumford type. The morphism $\mathrm{M}_{g}(\vec{l}) \rightarrow \overline{\mathrm{M}}_{g}$ is finite, flat, and is an isomorphism on the open dense substack $\mathrm{M}_{\mathrm{g}}$.

II. Any proper substack $\mathrm{X}$ of $\widetilde{\mathrm{M}}_{g}$ fitting in $\mathrm{M}_{g} \hookrightarrow \mathrm{X} \hookrightarrow \widetilde{\mathrm{M}}_{g}$ is isomorphic to $\mathrm{M}_{g}(\vec{l})$ for a suitable multiindex $\vec{l}$.

Proof. There is a natural surjective morphism

$$
\widetilde{\mathrm{M}}_{g} \rightarrow \overline{\mathrm{M}}_{g}
$$

which is the functor sending a twisted curve $\mathrm{C} \rightarrow X$ to the coarse space $|\mathrm{C}| \rightarrow X$. Note that $\mathrm{M}_{g}$ is dense in $\widetilde{\mathrm{M}}_{g}$ : by Theorem 2.4.6 any twisted curve $\mathrm{C} \rightarrow$ Spec $k$ can be realized as the special fibre of a twisted curve $C^{\prime}$ over a discrete valuation ring in such a way that the generic fibre is smooth.

Point (I) follows from [O107, Thm. 1.9] and, in particular, from the description of versal deformation spaces (2.4.7). The morphism $\mathrm{M}_{g}(\vec{l}) \rightarrow \overline{\mathrm{M}}_{g}$ is locally represented by the flat, finite, tame morphism of Deligne-Mumford type

$$
\left[(\operatorname{Spec} \widetilde{I}) / \boldsymbol{\mu}_{h_{1}} \times \cdots \times \boldsymbol{\mu}_{h_{m}}\right] \rightarrow \operatorname{Spec} I
$$

where $I$ is the versal deformation space of a point of $\overline{\mathrm{M}}_{g}, m$ is the number of nodes of the curve represented by such point, $\widetilde{I}=I\left[z_{1}, \ldots, z_{m}\right] /\left(z_{1}^{h_{1}}-t_{1}, \ldots, z_{m}^{h_{m}}-t_{m}\right)$ and $\boldsymbol{\mu}_{h_{1}} \times \cdots \times \boldsymbol{\mu}_{h_{m}}$ acts as $\left(\xi_{h_{1}}, \ldots, \xi_{h_{m}}\right) z_{i}=\xi_{h_{j}} z_{j}$ (note that the index $h_{j}$ depends on the type of the $j$ th node and $h_{j}=l_{i}$ if the $j$ th node is of type $i$ ). This means that $\mathrm{M}_{g}(\vec{l})$ is smooth, which is also shown in ACV03, $\S 3]$ and [AJ03, §3]. The fact that $\mathrm{M}_{g}(\vec{l})$ is irreducible is a consequence of the fact that $\mathrm{M}_{g}$ is dense on $\mathrm{M}_{g}(\vec{l})$.

We show (II). Let $X$ be a proper stack, which contains $M_{g}$ and is contained in $\widetilde{M}_{g}$. By restriction of $\widetilde{\mathrm{M}}_{g} \rightarrow \overline{\mathrm{M}}_{g}$ to $\mathrm{X}$, we obtain a morphism of proper stacks $\mathrm{f}: \mathrm{X} \rightarrow \overline{\mathrm{M}}_{g}$. Note that $\mathrm{M}_{g}$ is dense in $X$ and in $\bar{M}_{g}$. The valuative criterion of properness for $X$ and $\bar{M}_{g}$ implies that for any 
geometric point y: Spec $k \rightarrow \overline{\mathrm{M}}_{g}$ there exists a point $\mathrm{x}:$ Spec $k \rightarrow \mathrm{X}$ lifting $\mathrm{y}$. In fact $\mathrm{x}$ is unique. To see this, consider a versal deformation Spec $I \rightarrow \overline{\mathrm{M}}_{g}$ at $\mathrm{y}$. The base change of $\mathrm{X} \rightarrow \overline{\mathrm{M}}_{g}$ via $\operatorname{Spec} I \rightarrow \overline{\mathrm{M}}_{g}$ is a restriction of (4.1.5). The point $\mathrm{y}$ is in the locus $z_{1}=z_{2}=\cdots=z_{m}=0$ and admits only one lifting.

Now, we define positive indexes $\vec{l}=\left(l_{0}, l_{1}, \ldots, l_{|g / 2|}\right)$ such that $\mathrm{X}$ is isomorphic to $\mathrm{M}_{g}(\vec{l})$. Denote by $\mathrm{u}: \mathrm{U}\left(\overline{\mathrm{M}}_{g}\right) \rightarrow \overline{\mathrm{M}}_{g}$ the universal stable curve. Consider a geometric point $\mathrm{p}:$ Spec $k \rightarrow$ $\mathrm{U}\left(\overline{\mathrm{M}}_{g}\right)$, which is a node of type $i$. Let q: Spec $k \rightarrow \mathrm{X}$ be the unique morphism which lifts $\mathrm{u} \circ \mathrm{p}$. The object determined by $q$ is a twisted curve $C$, whose coarse space $|C|$ is represented by the point $\mathrm{u} \circ \mathrm{p}$. We define $l_{i}$ as the order of the stabilizer of $\mathrm{C}$ on the node $\mathrm{p}$. The index $l_{i}$ is locally constant on the (connected) substack of $\mathrm{U}\left(\overline{\mathrm{M}}_{g}\right)$ of nodes of type $i$. Therefore, $l_{i}$ only depends on $i$. This implies that the objets of $\mathbf{X}$ are $\vec{l}$-stable curves. Finally, the properness of $\mathbf{X}$ implies that any point Spec $k \rightarrow \mathrm{M}_{g}(\vec{l})$ lifts to $\mathrm{X}$. Since $\mathrm{M}_{g}(\vec{l})$ is regular, this suffices to show that $\mathrm{X}$ is isomorphic to $\mathrm{M}_{g}(\vec{l})$.

In fact, the stack $\mathrm{M}_{g}(\vec{l})$ admits an alternative description.

The first part of Olsson's proof of Theorem 2.4.6 in [O107] consists of constructing a functor from the category of twisted curves to the category of simple logarithmic extensions of the logarithmic structure $\mathcal{M}_{\Delta}$ on $\overline{\mathrm{M}}_{g}$ canonically associated to the boundary locus $\Delta=\overline{\mathrm{M}}_{g} \backslash \mathrm{M}_{g}$. Indeed, the boundary locus is a normal crossings divisor $\Delta=\sum_{i} \Delta_{i}$ where $\Delta_{i}$ is the full subcategory of stable curves $C \rightarrow X$ where the geometric fibres on every $x$ in $X$ are curves containing at least a node of type $i$ (recall that a logarithmic structure is canonically associated to any normal crossings divisor [Ka89, 1.5]). The second part of Olsson's proof shows that simple extensions of logarithmic structures form an algebraic stack.

We point out that, by [0107, Lem. 5,3], Olsson's functor can be regarded as an equivalence between the subcategory $\mathrm{M}_{g}(\vec{l})$ of $\vec{l}=\left(l_{0}, l_{1}, \ldots, l_{\lfloor g / 2\rfloor}\right)$-stable curves and the subcategory of simple extensions $\mathcal{M}_{\Delta} \rightarrow \mathcal{N}$ of locally free logarithmic structures inducing, at each geometric point $x:$ Spec $k \rightarrow X$, the commutative diagram

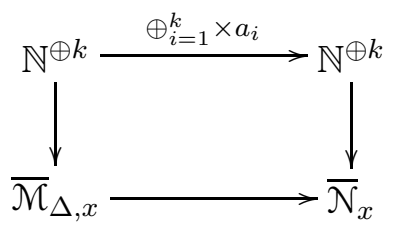

where $\{1, \ldots, k\}$ is the set of irreducible components of the local picture of $\Delta$ at $x$, and $a_{i}=l_{j}$ if $i \in\{1, \ldots, k\}$ corresponds to the component $\Delta_{j}$ in $\Delta$ (following Olsson we adopt the notation $\overline{\mathcal{M}}_{\Delta}=\mathcal{M}_{\Delta} / \mathcal{O}^{\times}, \overline{\mathcal{N}}=\mathcal{N} / \mathcal{O}^{\times}$and we refer to [MO05, Lem 4.2] for the proof of the canonical decomposition of $\overline{\mathcal{M}}_{\Delta, x} \cong \mathbb{N}^{\oplus k}$ ).

Now, we notice that this subcategory of logarithmic extensions is precisely the category used in Matsuki and Olsson's Generalization 2.2.5. In this way, we get the following statement. 
4.1.6 Theorem. For any multiindex $\left(l_{0}, l_{1}, \ldots, l_{\lfloor g / 2\rfloor}\right)$, we have the following isomorphism

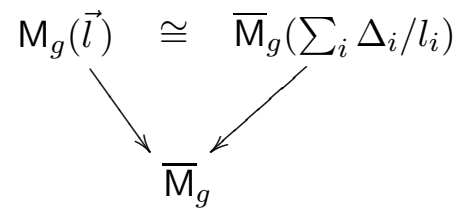

where $\mathrm{M}_{g}(\vec{l})$ is the stack of $\vec{l}$-stable curves and $\overline{\mathrm{M}}_{g}\left(\sum_{i} \Delta_{i} / l_{i}\right)$ is the stack of simple extensions of the logarithmic structure associated to $\sum_{i} \Delta_{i}$ with indexes $l_{i}$ in the sense of [MO05, (4.2.2)].

\subsection{The moduli stack of $r$ th roots on $\vec{l}$-stable curves}

Let $\mathrm{LB}_{g}$ be the category formed by pairs $(C \rightarrow X, M)$, where $C \rightarrow X$ is a smooth curve and $M$ is a line bundle on $C$. The stack $\mathrm{LB}_{g}$ is the category fibred over $\mathrm{M}_{g}$ whose fibre over a smooth curve $f: C \rightarrow X$ is the stack $\mathrm{LB}_{f}$. Let $\mathrm{F}$ be a line bundle on the universal curve of $\mathrm{M}_{g}$, whose relative degree is a multiple of $r$. Let $\mathrm{M}_{g}^{\mathrm{F}, r}$ be the fibred category whose fibre over $f: C \rightarrow X$ is $\mathrm{F}^{1 / r}$. By Proposition 3.1.3. $\mathrm{M}_{g}^{\mathrm{F}, r}$ is a Deligne-Mumford stack, étale over $\mathrm{M}_{g}$.

It is well known that $\mathrm{F}$ is a power $\omega^{\otimes k}$ of the relative dualizing sheaf modulo pullbacks from $\mathrm{M}_{g}$ (Enriques and Franchetta's conjecture, [Ha83] [Me87] [AC87]). In view of a compactification of $\mathrm{M}_{g}^{\mathrm{F}, r}$, we focus on the case

$$
\mathrm{F}=\omega^{\otimes k}
$$

and we assume

$$
(2 g-2) k \in r \mathbb{Z} .
$$

We extend $\mathrm{LB}_{g}$ to $\widetilde{\mathrm{M}}_{g}$ : we consider the category $\widetilde{\mathrm{LB}}_{g}$ formed by pairs $(\mathrm{C} \rightarrow X, \mathrm{M})$ where $\mathrm{C} \rightarrow X$ is a twisted curve and $\mathrm{M}$ is a line bundle on $\mathrm{C}$, Remark 3.1.1. We get the stack

$$
\widetilde{\mathrm{LB}}_{g} \rightarrow \widetilde{\mathrm{M}}_{g}
$$

By abuse of notation, we denote by $\mathrm{F}$ the power of the relative dualizing sheaf $\omega^{\otimes k}$ on the universal curve over $\widetilde{\mathrm{M}}_{g}$. Let $\widetilde{\mathrm{M}}_{g}^{\mathrm{F}, r}$ be the fibred category over $\widetilde{\mathrm{M}}_{g}$ whose fibre over $\mathrm{f}: \mathrm{C} \rightarrow X$ is the stack of $\mathrm{F}^{1 / r}$ of $r$ th roots of $\mathrm{F}$ on $\mathrm{C}$. In this way, the objects of $\widetilde{\mathrm{M}}_{g}^{\mathrm{F}, r}$ are triples $(\mathrm{C} \rightarrow X, \mathrm{~L}, \mathrm{j})$, where $C$ is a twisted curve, $L$ is a line bundle on $C$ and $j$ is an isomorphism $j: L^{\otimes r} \rightarrow F_{C}$. Morphisms from $(\mathrm{C} \rightarrow X, \mathrm{~L}, \mathrm{j})$ to $\left(\mathrm{C}^{\prime} \rightarrow X^{\prime}, \mathrm{L}^{\prime}, \mathrm{j}^{\prime}\right)$ are pairs $(\mathrm{m}, \mathrm{a})$ where $\mathrm{m}: \mathrm{C} \rightarrow \mathrm{C}^{\prime}$ is a morphism of twisted curves and a: $\mathrm{L} \rightarrow \mathrm{m}^{*} \mathrm{~L}^{\prime}$ is an isomorphism of line bundles, with $\mathrm{a}^{\otimes r}$ commuting with $\mathrm{j}$ and $\mathrm{j}^{\prime}$.

By Proposition 3.1.3, $\widetilde{\mathrm{M}}_{g}^{\mathrm{F}, r}$ is a Deligne-Mumford stack étale over $\widetilde{\mathrm{M}}_{g}$

$$
\widetilde{\mathrm{M}}_{g}^{\mathrm{F}, r} \rightarrow \widetilde{\mathrm{M}}_{g}
$$

which can be regarded as the projection on the second factor of the fibred product $\left(\widetilde{\mathrm{LB}}_{g}\right) \mathrm{k}_{r} \times_{\mathrm{F}} \widetilde{\mathrm{M}}_{g}$ 
fitting in

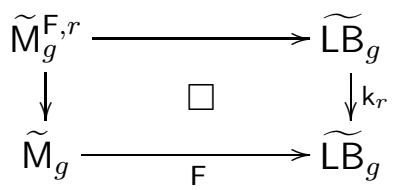

where $\mathrm{k}_{r}$ is induced by the $r$ th power in $\mathbb{G}_{m}$ and $\mathrm{F}=\omega^{\otimes k}$ is regarded as a section of (4.2.1).

We consider the étale morphism $\widetilde{\mathrm{M}}_{g}^{\mathrm{F}, r} \rightarrow \widetilde{\mathrm{M}}_{g}$. Its restriction to the stack $\mathrm{M}_{g}^{\mathrm{F}, r}$ of $r$ th roots of $\mathrm{F}$ on smooth curves $\mathrm{C} \rightarrow X$ forms a finite stack on $\mathrm{M}_{g}$ equipped with a torsor structure under the group stack $\mathrm{M}_{g}^{\mathcal{O}, r}$. On the other hand $\widetilde{\mathrm{M}}_{g}$ is not separated and so is $\widetilde{\mathrm{M}}_{g}^{\mathrm{F}, r}$. By Theorem 4.1.4, we can consider the base changes to all compactifications of $\mathrm{M}_{g}$ in $\widetilde{\mathrm{M}}_{g}$ via $\mathrm{M}_{g}(\vec{l}) \hookrightarrow \widetilde{\mathrm{M}}_{g}$ : we get $\mathrm{M}_{g}^{\mathrm{F}, r}(\vec{l}) \rightarrow \widetilde{\mathrm{M}}_{g}(\vec{l})$

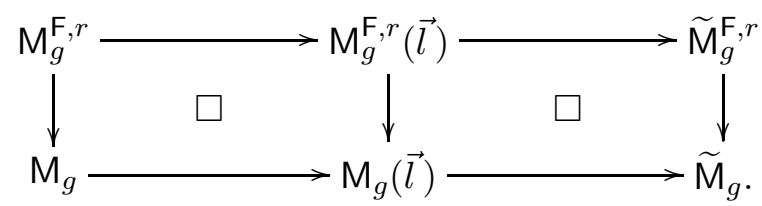

We now characterize the compactifications $\mathrm{M}_{g}^{\mathrm{F}, r}(\vec{l})$ for which the morphism to $\widetilde{\mathrm{M}}_{g}(\vec{l})$ is proper.

4.2.3 Theorem. For any $\mathrm{F}=\omega^{\otimes k}$, the category $\mathrm{M}_{g}^{\mathrm{F}, r}(\vec{l})$ is a smooth Deligne-Mumford algebraic stack, étale on $\mathrm{M}_{g}(\vec{l})$.

I. For $\mathrm{F}=\mathcal{O}$, the stack $\mathrm{M}_{g}^{\mathcal{O}, r}(\vec{l})$ is a finite group stack if and only if $r$ divides $l_{0}$.

II. For $\mathrm{F}=\omega$ and $2 g-2 \in r \mathbb{Z}$, the stack $\mathrm{M}_{g}^{\mathcal{O}, r}(\vec{l})$ is a finite group stack and $\mathrm{M}_{g}^{\omega, r}(\vec{l})$ is a finite torsor under $\mathrm{M}_{g}^{\mathcal{O}, r}(\vec{l})$ if and only if $r$ divides

$$
(2 i-1) l_{i} \text { for all } i \text {. }
$$

In this way, we obtain several compactifications of the stack $\mathrm{M}_{g}^{\omega, r}$ of smooth r-spin curves: for each $\vec{l}$ satisfying $l_{i}(2 i-1) \in r \mathbb{Z}$,

$$
\mathrm{M}_{g}^{\omega, r}(\vec{l}) \rightarrow \mathrm{M}_{g}(\vec{l})
$$

is the finite torsor of $r$-spin $\vec{l}$-stable curves.

III. More generally, for $\mathrm{F}=\omega^{\otimes k}$ and $(2 g-2) k \in r \mathbb{Z}$, the stack $\mathrm{M}_{g}^{\mathcal{O}, r}(\vec{l})$ is a finite group stack and $\mathrm{M}_{g}^{\mathrm{F}, r}(\vec{l})$ is a finite torsor under $\mathrm{M}_{g}^{\mathcal{O}, r}(\vec{l})$ if and only if $r$ divides

$$
l_{0} \quad \text { and } \quad(2 i-1) k l_{i}, \text { for } i>0 .
$$

Proof. It is enough to show (III). We check the numerical condition of Theorem 3.2 .2 for $\mathrm{F}=\omega^{\otimes k}$. Let e be a node of a twisted curve. If e is nonseparating, the condition amounts to require that $r$ divides \#(Aut(e)). If e is separating and of type $i$, then the numerical condition is that $r$ divides \#(Aut(e) $k(2 i-1)$. In this way, Theorem 3.2 .2 implies the claim.

4.2.4 Proposition. The category $\mathrm{M}_{g}^{\mathcal{O}, r}(\vec{l})$ is equivalent to the category of $\boldsymbol{\mu}_{r}$-torsors on $\vec{l}$-stable curves. In this way, as soon as $r$ divides $l_{0}$, the stack $\mathrm{M}_{g}^{\mathcal{O}, r}(\vec{l})$ is a compactification of the stack of $\boldsymbol{\mu}_{r}$-torsors on smooth curves. 
Proof. There is a natural functor from $\boldsymbol{\mu}_{r}$-torsors to $r$-torsion line bundles. Given a torsor $\mathrm{T}$ on $\mathrm{C}$ with an action of $\boldsymbol{\mu}_{r}$, consider $\mathrm{P}=\mathrm{T} \times{ }_{\mathrm{C}} \mathbb{A}^{1}$ over $\mathrm{C}$. Note that $\boldsymbol{\mu}_{r}$ acts on both factors and the diagonal action on the fibre product is free. The quotient $\mathrm{P} / \boldsymbol{\mu}_{r}$ yields an $r$-torsion line bundle on $\mathrm{C}$. The functor is essentially surjective and fully faithful.

4.2.5 Remark. For each object of $\mathrm{M}_{g}^{\mathrm{F}, r}(\vec{l})$ there is an injection of $\boldsymbol{\mu}_{r}$ in the automorphism group (the $r$ th roots of unity act by multiplication along the fibres of the line bundle). The rigidification of $\mathrm{M}_{g}^{\mathrm{F}, r}(\vec{l})$ along the group scheme $\boldsymbol{\mu}_{r}$ yields a representable cover $\mathrm{H}(\mathrm{F})^{\boldsymbol{\mu}_{r}}$ of $\mathrm{M}_{g}(\vec{l})$, see Proposition 3.1.5. In this way, the morphism $\mathrm{M}_{g}^{\mathrm{F}, r}(\vec{l}) \rightarrow \mathrm{M}_{g}(\vec{l})$ factors as

$$
\mathrm{M}_{g}^{\mathrm{F}, r}(\vec{l}) \rightarrow \mathrm{H}(\mathrm{F})^{\boldsymbol{\mu}_{r}} \rightarrow \mathrm{M}_{g}(\vec{l})
$$

where $\mathrm{H}(\mathrm{F})^{\boldsymbol{\mu}_{r}} \rightarrow \mathrm{M}_{g}(\vec{l})$ is a representable cover of degree $r^{2 g}$ and $\mathrm{M}_{g}^{\mathrm{F}, r}(\vec{l}) \rightarrow \mathrm{H}(\mathrm{F})^{\boldsymbol{\mu}_{r}}$ is an étale $\boldsymbol{\mu}_{r}$-gerbe. By Proposition 3.1.5 and Proposition 3.1.6, as soon as $\vec{l}$ satisfies $l_{0} \in r \mathbb{Z}$ and $(2 i-1) k l_{i} \in r \mathbb{Z}$ for $i>0$ the base change of $\mathrm{H}(\mathcal{O})^{\boldsymbol{\mu}_{r}} \rightarrow \mathrm{M}_{g}(\vec{l})$ and of $\mathrm{H}(\mathrm{F})^{\boldsymbol{\mu}_{r}} \rightarrow \mathrm{M}_{g}(\vec{l})$ with respect to a morphism from a scheme $X$ to $\mathrm{M}_{g}(\vec{l})$ yields a finite group $X$-scheme $G_{X}$ and a finite torsor $T_{X}$ under $G_{X}$.

4.2.6 Remark ( $\lambda$-stable curves). Note that Theorem 4.2.3 is automatically satisfied if $l_{i}=\lambda$ for all $i$ and $\lambda$ is a multiple of $r$. In this case, we write " $\lambda$-stable" instead $\vec{l}$-stable.

4.2.7 Remark ( $n$-pointed curves). Olsson's results [Ol07, Thm. 1.9] are formulated in terms of $n$ pointed curves; therefore, the category $\widetilde{\mathrm{M}}_{g, n}$ of twisted curves, with $n$ ordered distinct markings $\sigma_{1}, \ldots, \sigma_{n}$ in the smooth locus, is a Deligne-Mumford stack. We point out that our method produces proper stacks of $r$ th roots of any line bundle $\mathrm{F}=\omega^{\otimes k}\left(\sum_{i}-h_{i}\left[\sigma_{i}\right]\right)$ on the universal twisted curve over $\widetilde{\mathrm{M}}_{g, n}$ for all integers $k, h_{1}, \ldots, h_{n}$ satisfying $(2 g-2) k-\sum_{i} h_{i} \in r \mathbb{Z}$. (The case $k=1$ is relevant to Witten's conjecture [Wi93]). This happens by Corollary 3.0 .8 and the fact that $\mathrm{F}=\omega^{\otimes k}$ is a pullback from the universal (scheme-theoretic) stable $n$-pointed curve over $\widetilde{\mathrm{M}}_{g, n}$, Proposition 2.5.1, In general, Corollary 3.0.8 yields the following statement.

4.2.8 Corollary. Let $\mathrm{F}$ be a line bundle on the universal twisted n-pointed curve isomorphic to a pullback from the universal stable n-pointed curve. Let $\widetilde{\mathrm{M}}_{g, n}^{\mathrm{F}, r}$ be the fibred category on $\widetilde{\mathrm{M}}_{g, n}$ of $r$ th roots of $\mathrm{F}$ on twisted curves. It is a stack, étale on $\widetilde{\mathrm{M}}_{g, n}$. It is nonempty, as long as we assume that the relative degree of $\mathrm{F}$ is a multiple of $r$.

For any $\lambda \in \mathbb{Z}$, the stack of $\lambda$-stable n-pointed curves $\mathrm{M}_{g, n}(\lambda)$ is smooth, irreducible, and proper. If $r$ divides $\lambda$, the stack $\mathrm{M}_{g, n}^{\mathcal{O}, r}(\lambda)$ of $r$-torsion line bundles on $\lambda$-stable $n$-pointed curves is a finite group stack and the stack $\mathrm{M}_{g, n}^{\mathrm{F}, r}(\lambda)$ of rth roots of $\mathrm{F}$ on $\lambda$-stable curves is a finite torsor under the group stack $\mathrm{M}_{g, n}^{\mathcal{O}, r}(\lambda)$.

In this way, we obtain several compactifications of the stack $\mathrm{M}_{g, n}^{\omega, r}$ of smooth r-spin n-pointed curves: for each $\lambda \in r \mathbb{Z}$,

$$
\mathrm{M}_{g, n}^{\omega, r}(\lambda) \rightarrow \mathrm{M}_{g, n}(\lambda)
$$

is the finite torsor of $r$-spin $n$-pointed $\lambda$-stable curves.

4.2.9 Remark. Let $r_{1}$ and $r_{2}$ be positive and coprime integers. In [JKV00, $\S 4$, Rem. 4.11], it is noted that the functor $L \mapsto\left(L^{\otimes r_{2}}, L^{\otimes r_{1}}\right)$ is an isomorphism $\mathrm{M}_{g}^{\mathrm{F}, r_{1} r_{2}} \cong \mathrm{M}_{g}^{\mathrm{F}, r_{1}} \times \mathrm{M}_{g} \mathrm{M}_{g}^{\mathrm{F}, r_{2}}$, that does not extend to the compactifications given in the existing literature. With our formalism the isomorphism extends immediately (we omit markings for simplicity). 
4.2.10 Proposition (roots of two coprime orders). Let $r_{1}$ and $r_{2}$ be positive and coprime integers. Set $\lambda=r_{1} r_{2}$. The functor $\mathrm{L} \mapsto\left(\mathrm{L}^{\otimes r_{2}}, \mathrm{~L}^{\otimes r_{1}}\right)$ is an isomorphism of stacks

$$
\mathrm{M}_{g}^{\mathrm{F}, r_{1} r_{2}}(\lambda) \cong \mathrm{M}_{g}^{\mathrm{F}, r_{1}}(\lambda) \times \mathrm{M}_{g}(\lambda) \mathrm{M}_{g}^{\mathrm{F}, r_{2}}(\lambda)
$$

Proof. The inverse functor is $\left(\mathrm{L}_{1}, \mathrm{~L}_{2}\right) \mapsto \mathrm{L}_{1}^{\otimes h_{2}} \otimes \mathrm{L}_{2}^{\otimes h_{1}}$ for $h_{1}$ and $h_{2}$ satisfying $h_{1} r_{1}+h_{2} r_{2}=1$. Indeed, we have $\left(\mathrm{L}_{1}^{\otimes h_{2}} \otimes \mathrm{L}_{2}^{\otimes h_{1}}\right)^{\otimes r_{1} r_{2}}=\mathrm{L}_{1}^{\otimes r_{1} r_{2} h_{2}} \otimes \mathrm{L}_{2}^{\otimes r_{2} r_{1} h_{1}} \cong \mathrm{F}^{\otimes h_{1} r_{1}+h_{2} r_{2}}=\mathrm{F}$.

4.2.11 Example $(g=1$ and $n=1)$. Consider the twisted curve $\mathrm{C}=\left[E / \boldsymbol{\mu}_{2}\right]$, where $E$ is equal to $\mathbb{P}^{1} /(0 \sim \infty)$ and $\boldsymbol{\mu}_{2}$ acts by change of sign. We now exhibit the four distinct square roots of $\omega_{\mathrm{C}}$ up to isomorphism. If we denote by $\times$ a smooth point of $\mathrm{C}$, we can regard this example as a check that the fibre of $\mathrm{M}_{1,1}^{\omega / 2}(2) \rightarrow \mathrm{M}_{1,1}(2)$ over the geometric point representing $(\mathrm{C}, \mathrm{x})$ actually contains 4 distinct geometric points.

Note that $\omega_{C}$ is trivial, so we are actually looking for square roots of $\mathcal{O}_{C}$. Consider the normalization $\mathrm{C}^{\nu} \rightarrow \mathrm{C}$, which is isomorphic to $\left[\mathbb{P}^{1} / \boldsymbol{\mu}_{2}\right]$. On $\mathrm{C}^{\nu}$ there are 2 roots of $\mathcal{O}$ : the line bundle $\mathcal{O}_{\mathbb{P}^{1}}$ with trivial $\boldsymbol{\mu}_{2^{-a c t i o n}}$ on the fibres; and the line bundle $\mathcal{O}_{\mathbb{P}^{1}}$ with $\boldsymbol{\mu}_{2^{2}}$-action on the fibres given by $t \mapsto-t$. Each of these line bundles descend to $E$ to form a root of $\mathcal{O}_{E}$ in exactly two nonisomorphic ways. Therefore, on $\mathrm{C}$, we get four square roots $\mathrm{L}^{++}, \mathrm{L}^{+-}, \mathrm{L}^{-+}$, and $\mathrm{L}^{--}$of $\omega_{\mathrm{C}}$ up to isomorphism.

Note that the line bundles $L^{\sigma, \tau}$ above, are 2-torsion line bundles and can be regarded as the 2-torsion subgroups of $\mathrm{Pic}^{\boldsymbol{\mu}_{2}}(E)$, the group of $\boldsymbol{\mu}_{2}$-linearized line bundles. Their geometric realizations are stacks fibred over $C$, which we describe explicitly. Let $W^{+}$be the line bundle on $E$, obtained from $\mathbb{P}^{1} \times \mathbb{A}^{1}$ by glueing the lines over 0 and $\infty$ via $(0, t) \sim(\infty, t)$ and let $W^{-}$be the line bundle on $E$ obtained from $\mathbb{P}^{1} \times \mathbb{A}^{1}$ by glueing the lines over 0 and $\infty$ via $(0, t) \sim(\infty,-t)$. The geometric bundles over $\mathrm{C}$ associated to $\mathrm{L}^{\sigma, \tau}$ for $\sigma, \tau \in\{+,-\}$ is the quotient stack $\left[W^{\tau} / \boldsymbol{\mu}_{2}\right]$ with $\boldsymbol{\mu}_{2}$ acting as $t \mapsto \sigma t$ on the fibres.

Since $\omega_{\mathrm{C}}$ is isomorphic to $\mathcal{O}$, following Proposition 4.2.4, we also provide a concrete description of the line bundles above in terms of $\boldsymbol{\mu}_{2}$-torsors on $\mathrm{C}$. We exhibit four distinct representable 2 -folded étale covers of $C$. Note that some points of the covering stack D over $C$ might have nontrivial stabilizer $\boldsymbol{\mu}_{2}$ : in the drawings we adopt the convention of marking them with a black circle. The labelling $\mathrm{D}^{++}, \mathrm{D}^{+-}, \mathrm{D}^{-+}$, and $\mathrm{D}^{--}$matches the above notations for the line bundles.

1. The cover $\mathrm{D}^{++}$. It is $\mathrm{D}^{++}=\mathrm{C} \times \boldsymbol{\mu}_{2}$ on which $\boldsymbol{\mu}_{2}$ acts as id $\times \xi_{2}$. The morphism $\mathrm{p}$ is the projection to the first factor.

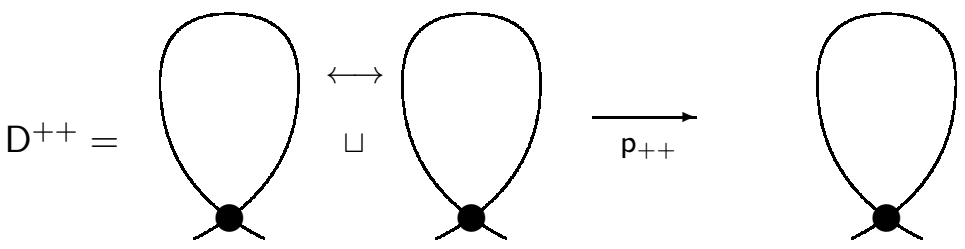

2. The cover $\mathrm{D}^{+-}$. Take $\left[\mathbb{P}^{1} / \pm\right] \times \boldsymbol{\mu}_{2}$ modulo the relation $(0, \sigma) \sim(\infty,-\sigma)$, for all $\sigma \in \boldsymbol{\mu}_{2}$. The $\boldsymbol{\mu}_{2}$-action is generated by id $\times \xi_{2}$ and the morphism $\mathrm{p}$ is the projection to the first factor. 


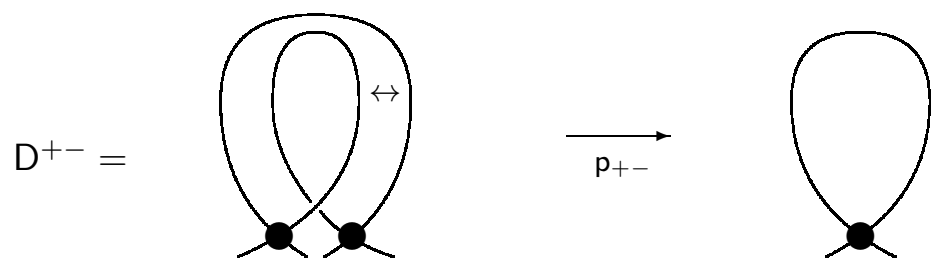

3. The cover $\mathrm{D}^{-+}$. Take the étale atlas $E=\mathbb{P}^{1} /(0 \sim \infty)$ of $\mathrm{C}$. The $\boldsymbol{\mu}_{2}$-action is the change of sign on $\mathbb{P}^{1}$. The morphism $\mathrm{p}$ is $E \rightarrow\left[E / \boldsymbol{\mu}_{2}\right]$. Note that, the local picture of $\mathrm{p}$ at the node is given by $(z, w) \mapsto(z, w)$ on $\{z w=0\} \rightarrow\left[\left\{z^{\prime} w^{\prime}=0\right\} / \boldsymbol{\mu}_{2}\right]$.

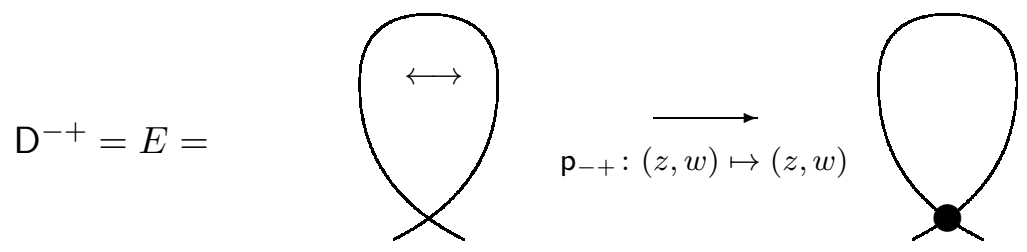

4. The cover $\mathrm{D}^{--}$. Take $E=\mathbb{P}^{1} /(0 \sim \infty)$ with $\boldsymbol{\mu}_{2}$ acting by change of sign as above. On the smooth locus $E^{\mathrm{sm}} \longrightarrow \mathrm{C}^{\mathrm{sm}}$ the morphism $\mathrm{p}$ is $x \mapsto x^{2}$. On the other hand, we define $\mathrm{p}$ so that its local picture at the node $\{z w=0\} \rightarrow\left[\left\{z^{\prime} w^{\prime}=0\right\} / \boldsymbol{\mu}_{2}\right]$ is given by $(z, w) \mapsto(z,-w)$.

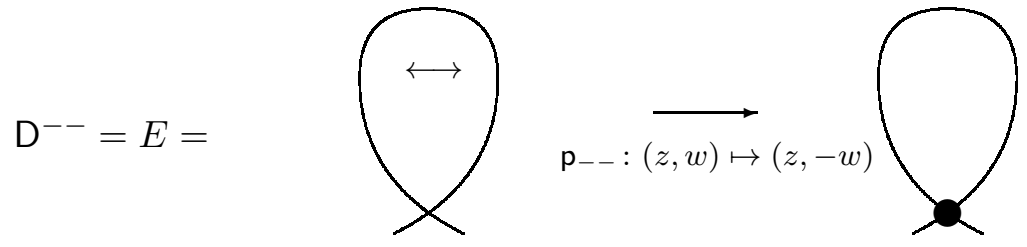

This completes the check that the fibre of $M_{1,1}^{\omega / 2}(2) \rightarrow M_{1,1}(2)$ over the curve $(C, x)$ in $M_{1,1}(2)$ is the 0 -dimensional stack given by 4 disjoint copies of $\mathrm{B}(\mathbb{Z} / 2 \mathbb{Z}$ ) (recall that each object has a nontrivial automorphism acting by multiplication by -1 along the fibre of the line bundle).

One can ask a natural question at this point: what is the fibre of the corresponding morphism between coarse spaces over the closed point corresponding to $(C, x)$ in the moduli space of stable curves? In order to answer this question one should note that the only automorphism of $(C, x)$ that acts nontrivially on the objects of $\mathrm{M}_{1,1}^{\omega / 2}(2)$ is the automorphism $\mathrm{g}$ of order 2 generating $\operatorname{Aut}(\mathrm{C},|\mathrm{C}|)$. Then, by Proposition 2.5.3, we note that the action of $\mathrm{g}$ fixes $\mathrm{L}^{++}$and $\mathrm{L}^{+-}$and identifies the objects $\mathrm{L}^{-+}$and $\mathrm{L}^{--}$. Indeed the formula in Proposition 2.5 .3 can be written as

$$
\begin{aligned}
& \mathrm{g}^{*} \mathrm{~L}^{++}=\mathrm{L}^{++} \otimes \mathrm{L}^{++}=\mathrm{L}^{++}, \\
& \mathrm{g}^{*} \mathrm{~L}^{+-}=\mathrm{L}^{+-} \otimes \mathrm{L}^{++}=\mathrm{L}^{+-}, \\
& \mathrm{g}^{*} \mathrm{~L}^{-+}=\mathrm{L}^{-+} \otimes \mathrm{L}^{+-}=\mathrm{L}^{--} .
\end{aligned}
$$

Therefore, the fibre is the disjoint union of two reduced point and a third point of length two over the coarse moduli space of stable curves.

4.2.12 Remark. By Proposition 2.5.3, this analysis can be generalized to any integer $r>2$. For simplicity, we consider $r$ prime. Let $\mathrm{C}$ be the $r$-stable curve on the 1-pointed nodal curve $\mathbb{P}^{1} /(0 \sim \infty)$. The group $\operatorname{Aut}(\mathrm{C},|\mathrm{C}|)=\boldsymbol{\mu}_{r}$ acts freely on the $\left(r^{2}-r\right)$ spin structures that are not pullbacks from $|\mathrm{C}|$. This means that the fibre of the morphism to $\overline{\mathrm{M}}_{1,1}$ contains 1 
point representing the trivial $r$-spin structures and $2 r-2$ points representing nontrivial $r$-spin structures, half of which are pullbacks from the coarse space. After the identifications induced by the hyperelliptic involution we get

there are exactly $r-1$ nontrivial $r$-spin $r$-stable curves over $\mathbb{P}^{1} /(0 \sim \infty)$.

This allows to picture the coarse space of nontrivial $r$-spin curves of genus 1 , with 1 marking. This leads us to point out the following counterexample to Conjecture 4.2 .1 of [Ja01, which predicts that the Picard group of the stack of smooth $r$-spin 1-pointed curves of genus 1 is finite. 4.2.14 Example. The space $N_{r}$ of nontrivial $r$-stable $r$-spin curves is a curve covering $\left|\mathrm{M}_{1,1}\right|$ : indeed it is an $\left(r^{2}-1\right) / 2$-fold cover of the projective line. For instance, fix $r=11$; then $N_{r} \rightarrow\left|\mathrm{M}_{1,1}\right|$ has degree 60 . Over the two curves with extra automorphisms there are respectively $\left(r^{2}-1\right) / 4=30$ and $\left(r^{2}-1\right) / 6=20$ spin structures. By (4.2.13), there are exactly $r-1=10$ singular spin curves. Then, by the Riemann-Hurwitz formula, the Euler-Poincaré characteristic is $\chi\left(N_{r}\right)=0$. In this way, $N_{r}$ is a genus- 1 curve. The moduli stack of nontrivial $r$-spin structures on smooth 1-pointed genus-1 curves is a stack over the genus-1 curve $N_{r}$ minus a finite number of points. Its Picard group cannot be finite. A similar computation using (4.2.13) shows that for any prime integer $r>3$ we have

$$
g\left(N_{r}\right)=(r-5)(r-7) / 24
$$

\subsection{The relation with Abramovich and Jarvis's compactification}

The compactifications [Ja00] and [AJ03] adopt two different methods but are isomorphic, [AJ03, Prop. 4.3.1]). We restate the construction. We use systematically the equivalence between line bundles on a stack $\mathrm{X}$ and morphisms $\mathrm{X} \rightarrow \mathrm{B} \mathbb{G}_{m}$.

The compactification $\mathrm{B}_{g, n}\left(\mathrm{~B} \mathbb{G}_{m}, \omega_{\text {log }}^{1 / r}\right)$ introduced in [AJ03] is the following category. An object is the datum of a 1-commutative diagram

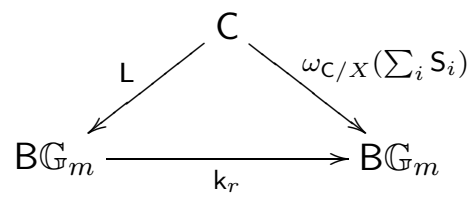

where $\mathrm{k}_{r}$ is induced by the homomorphism $t \mapsto t^{r}$ and the following conditions are satisfied.

1. The stack $C$ is of Deligne-Mumford type, flat of relative dimension 1 with nodal singularities over $X$.

2. The stacks $S_{1}, \ldots, S_{n}$ are closed disjoint substacks of $C^{\mathrm{sm}}$ and étale gerbes over $X$.

3. The corresponding coarse spaces $|\mathrm{C}|,\left|\mathrm{S}_{1}\right|, \ldots,\left|\mathrm{S}_{n}\right|$ form a proper, $n$-pointed, nodal curve over $X$, and $\pi: C \rightarrow|C|$ is an isomorphism away from the nodes and the stacks $S_{i}$.

4. At a node $\mathrm{p}$ in $\mathrm{C}$, for a suitable integer $l$, the local picture is given by $\left[V / \boldsymbol{\mu}_{l}\right]$, where, for some $t \in T, V$ is $\operatorname{Spec}(T[z, w] /(z w-t))$ and $\boldsymbol{\mu}_{l}$ acts as $(z, w) \mapsto\left(\xi_{l} z, \xi_{l}^{-1} w\right)$.

5. The morphism $L$ is representable. 
A morphism is a 1-commutative diagram

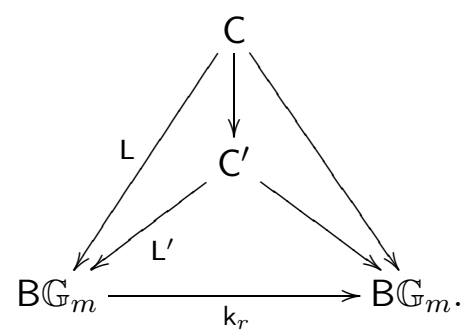

As usual, morphism are considered up to 2-isomorphisms (see Lemma 2.2.1).

In fact, we show that $\mathrm{B}_{g, n}\left(\mathrm{~B} \mathbb{G}_{m}, \omega_{\text {log }}^{1 / r}\right)$ is a compactification of

$$
\bigsqcup_{0 \leq h_{i}<r} \mathbf{M}_{g, n}^{\omega(\boldsymbol{h}), r},
$$

where $\omega(\boldsymbol{h}):=\omega\left(-\sum_{i} h_{i} S_{i}\right)$ is the relative dualizing sheaf on the universal curve over $\mathrm{M}_{g, n}$ twisted by the divisors $S_{n}$ determining the $i$ th marking.

4.3.1 Definition (faithful line bundles). A line bundle $\mathrm{M}$ on a twisted curve $\mathrm{C} \rightarrow X$ is faithful if it satisfies the following condition: for each node e on $C$ the action of Aut(e) on $M_{e}$ is faithful.

4.3.2 Remark. In the case of a line bundle $\mathrm{M}$ whose $r$ th tensor power is the pullback of a line bundle $F$ on the coarse space, for any $g \in \operatorname{Aut}(\mathrm{e})$ the element $g^{r}$ acts as the identity on $\mathrm{M}_{\mathrm{e}}$. Therefore, the fact that $\mathrm{M}$ is faithful implies that \#(Aut(e)) divides $r$.

4.3.3 Proposition. There is a finite and surjective morphism

$$
\bigsqcup_{0 \leq h_{i}<r} \mathrm{M}_{g, n}^{\omega(\boldsymbol{h}), r}(r) \longrightarrow \mathrm{B}_{g, n}\left(\mathrm{~B} \mathbb{G}_{m}, \omega_{l o g}^{1 / r}\right)
$$

where $\mathbf{M}_{g, n}^{\omega(\boldsymbol{h}), r}(r)$ is the stack of $r$ th roots of $\omega(\boldsymbol{h})=\omega\left(-\sum_{i} h_{i} S_{i}\right)$ over n-pointed r-stable curves.

The morphism (4.3.4) has degree one, but, in general, it is not an isomorphism. Indeed, consider a point $\mathrm{x}$ in $\mathrm{M}_{g, n}^{\omega(\boldsymbol{h}), r}(r)$ and its image $\mathrm{y}$ in $\mathrm{B}_{g, n}\left(\mathrm{~B}_{\mathbb{G}_{m}}, \omega_{l o g}^{1 / r}\right)$. We have

$$
\#(\operatorname{Aut}(\mathrm{x})) / \#(\operatorname{Aut}(\mathrm{y}))=r^{m} / \prod_{i=1}^{m} d_{i}
$$

where $d_{1}, \ldots, d_{m}$ are the orders of the automorphism groups of the nodes $\mathrm{e}_{1}, \ldots, \mathrm{e}_{m}$ of the twisted curve corresponding to $\mathrm{y}$.

Proof. There is an equivalence of categories

$$
\mathrm{B}_{g, n}\left(\mathrm{~B} \mathbb{G}_{m}, \omega_{l o g}^{1 / r}\right) \longrightarrow \bigsqcup_{0 \leq h_{i}<r} \mathrm{P}\left(h_{1}, \ldots, h_{n}\right),
$$

where $\mathrm{P}\left(h_{1}, \ldots, h_{n}\right)$ is the category on $\widetilde{\mathrm{M}}_{g, n}$ of faithful $r$ th roots of the line bundle $\omega(\boldsymbol{h})=$ $\omega\left(-\sum_{i} h_{i} S_{i}\right)$ on twisted curves. We prove the equivalence.

First, we need to introduce a decomposition of $\mathrm{B}_{g, n}\left(\mathrm{~B} \mathbb{G}_{m}, \omega_{l o g}^{1 / r}\right)$ into connected components $\mathrm{P}(\vec{l}, \vec{m})$. The local picture of a line bundle $\mathrm{L}$ on $\mathrm{C}$ at a point $\mathrm{p}: \operatorname{Spec} k \rightarrow \mathrm{S}_{i} \hookrightarrow \mathrm{C}$ is given, for 
suitable indexes $l_{i}$ and $m_{i}$, by a $\boldsymbol{\mu}_{l_{i}}$-equivariant line bundle $\mathrm{W}$ on $\left[V / \boldsymbol{\mu}_{l_{i}}\right]$ where $V$ is $\operatorname{Spec} T[z]$, the action is $z \mapsto \xi_{l_{i}} z$, and $\mathrm{W}$ is linearized by the character $\xi_{l_{i}} \mapsto \xi_{l_{i}}^{m_{i}}$. The coefficients $l_{i}$ and $m_{i}$ induce locally constant morphisms I, $\mathrm{m}: \mathrm{B}_{g, n}\left(\mathrm{~B} \mathbb{G}_{m}, \omega_{l o g}^{1 / r}\right) \rightarrow \mathbb{Z}^{n}$ with $0 \leq \mathrm{m}<\mathrm{I}$. Note also that the representability condition on $\mathrm{L}$ implies that $\mathrm{I}$ and $\mathrm{m}$ are coprime on each coordinate of $\mathbb{Z}^{n}$. The values taken by $\mathrm{I}$ and $\mathrm{m}$ determine a decomposition of $\mathrm{B}_{g, n}\left(\mathrm{~B} \mathbb{G}_{m}, \omega_{l o g}^{1 / r}\right)$ as the disjoint union of substacks $\mathrm{P}(\vec{l}, \vec{m})$ where the vectors $\vec{l}=\left(l_{i}\right), \vec{m}=\left(m_{i}\right) \in \mathbb{Z}^{n}$ satisfy $0 \leq \vec{l}<\vec{m}$ and $\operatorname{hcf}\left\{m_{i}, l_{i}\right\}=1$ for any $i$.

Second, there is an equivalence of category between $\mathrm{P}(\vec{l}, \vec{m})$ and the stack $\mathrm{P}\left(h_{1}, \ldots, h_{n}\right)$ for $h_{i}=r m_{i} / l_{i}-1$. By [Ca07, Thm. 4.1] and [O107, Thm. 1.8], there is an equivalence between the category of twisted curves equipped with $n$ distinct sections

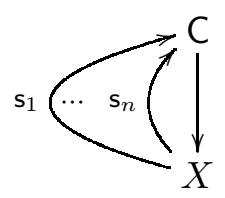

in the smooth locus and the category of Deligne-Mumford stacks $\mathrm{C} \rightarrow X$ satisfying conditions (1-4) in the definition of $\mathrm{B}_{g, n}\left(\mathrm{~B} \mathbb{G}_{m}, \omega_{\text {log }}^{1 / r}\right)$, where we require that the automorphism groups have order $l_{i}$ over the points of $S_{i}$. The functor sends $\left(\mathrm{C} \rightarrow X, \mathrm{~s}_{1}, \ldots, \mathrm{s}_{2}\right)$ to the stack $\mathrm{C} \times|\mathrm{C}| \mathrm{D}$, where we set $\mathrm{D}=|\mathrm{C}|\left[S_{1} / l_{1}\right] \times{ }_{|\mathrm{C}|} \cdots \times{ }_{|\mathrm{C}|}|\mathrm{C}|\left[S_{n} / l_{n}\right]$ for $S_{i}=\mathrm{s}_{i}(X)$. Note that the latter stack is equipped with a natural projection to $\mathrm{C}$. For $h_{i}=r m_{i} / l_{i}-1$, we have a functor $\mathrm{P}\left(h_{1}, \ldots, h_{n}\right) \rightarrow \mathrm{P}(\vec{l}, \vec{m})$ induced by pushforward via the projection. Indeed, the pushforward of line bundles on $\mathrm{D}$ is a line bundle on $|\mathrm{C}|$, see [AJ03, §3] or [Ca07]. In this way, the functor lands in $\mathrm{P}\left(h_{1}, \ldots, h_{n}\right)$ (the representability assumption at the nodes is equivalent to requiring that the $r$ th root is faithful at the nodes). The inverse functor is induced by pullback and tensorization with the tautological line bundles $\mathrm{M}_{i}^{\otimes m_{i}}$, which are defined on D and satisfy $\mathrm{M}_{i}^{\otimes l_{i}}=\mathcal{O}\left(S_{i}\right)$.

The morphism (4.3.4) is the composite of $\mathrm{P}\left(h_{1}, \ldots, h_{n}\right) \rightarrow \mathrm{P}(\vec{l}, \vec{m})$ and the disjoint union of finite and surjective morphisms of stacks

$$
\mathrm{M}_{g, n}^{\omega(h), r}(r) \rightarrow \mathrm{P}\left(h_{1}, \ldots h_{n}\right)
$$

whose restriction to the open and dense substack $\mathrm{M}_{g, n}^{\omega(\boldsymbol{h}), r}$ is the identity and whose corresponding morphism between coarse spaces is an isomorphism. We define the morphisms (4.3.5) here below.

The functor $\mathrm{M}_{g, n}^{\omega(\boldsymbol{h}), r}(r) \rightarrow \mathrm{P}\left(h_{1}, \ldots h_{n}\right)$, sends the morphism of stacks $\mathrm{C} \stackrel{L}{\rightarrow} \mathrm{B} \mathbb{G}_{m}$ to the corresponding representable morphism $\mathrm{C}^{\prime} \stackrel{L^{\prime}}{\rightarrow} \mathrm{B} \mathbb{G}_{m}$ making $\mathrm{C} \rightarrow \mathrm{C}^{\prime} \rightarrow \mathrm{B} \mathbb{G}_{m}$ the the "relative moduli space" in the sense of [ACV03, 5.2.4,(c)]. By means of the weak valuative criterion it is easy to see that this functor defines a surjection (each geometric point is lifted as in the proof of Theorem 4.1.4). The functor sending the object determined by $C$ and $L$ to the object determined by $C^{\prime}$ and $L^{\prime}$ is not an isomorphism in general. Indeed, the ratio of the orders of the automorphism groups at the two objects is $\#\left(\operatorname{Aut}\left(\mathrm{C}, \mathrm{C}^{\prime}\right)\right)$, where $\operatorname{Aut}\left(\mathrm{C}, \mathrm{C}^{\prime}\right)$ denotes the group of automorphisms of $C$ that fix $C^{\prime}$ (this happens because $L$ is the pullback of $L^{\prime}$ via $C \rightarrow C^{\prime}$ ). By Theorem 2.4.8, the order of $\operatorname{Aut}\left(\mathrm{C}, \mathrm{C}^{\prime}\right)$ is equal to $r^{m} / \prod_{i=1}^{m} d_{i}$, if we denote by $d_{1}, \ldots, d_{m}$ the orders of the automorphism groups of the nodes $\mathrm{e}_{1}, \ldots, \mathrm{e}_{m}$ of $\mathrm{C}^{\prime}$. 
4.3.6 Remark (the Witten top Chern class). The Witten top Chern class is a rational Chow cohomology class which plays a crucial role in the definition of the relevant numerical invariants in Witten's conjecture Wi93]. Although the new compactifications are not isomorphic to the preexisting one, the surjective morphism above yields an isomorphism between the coarse spaces. This implies that the rational Chow rings are isomorphic, see for example [Kr99].

There are two equivalent formulations [PV01 and [Ch06] of the construction of the Witten top Chern class and they both use the universal stable $r$-spin structure of Jarvis's compactification [Ja00], which is a sheaf of rank 1 rather than an invertible sheaf. Nevertheless they can be applied without modification to the new compactifications and yield the same class after the identification of the rational Chow cohomology rings.

4.3.7 Proposition. The Witten top Chern class functor defined in [PV01] and [Ch06] yields a class $c_{\mathrm{W}}$ in the rational cohomology of $\mathrm{M}_{g, n}^{\omega(\boldsymbol{h}), r}(r)$ as well as a class $\bar{c}_{\mathrm{W}}$ in the rational cohomology of Abramovich and Jarvis's compactification. The outputs are compatible in the sense that $c_{\mathrm{W}}$ is a pullback of $\bar{c}_{\mathrm{W}}$ via the surjective morphism of degree one exhibited in Proposition 4.3.3.

Proof. To see this, note that both constructions start from a datum in the derived category which is obtained by pushing Jarvis's universal $r$ th root $\mathcal{L}$ and the universal homomorphism $f$ along the universal stable curve. The morphism between our compactification and the compactification of Abramovich and Jarvis induces a morphism between the universal twisted curve on $\mathrm{M}_{g, n}^{\omega(\boldsymbol{h}), r}(r)$ and the universal stable curve on Jarvis's compactification. We only need to check that the pushforward to the universal stable curve yields the universal sheaf-theoretic stable $r$-spin structure $(\mathcal{L}, f)$ of Jarvis's construction [Ja00]. The proof of this fact can be found in [AJ03, $§ 3, \S 4.3]$, which applies verbatim to our setting.

\section{A Appendix. The stack $L B_{f}$}

We analyse the category $\mathrm{LB}_{\mathrm{f}}$ of line bundles on a flat and proper morphism $\mathrm{f}: \mathrm{Y} \rightarrow X$, where $\mathrm{Y}$ is a tame stack of Deligne-Mumford type, and $|\mathrm{f}|:|\mathrm{Y}| \rightarrow X$ is flat on $X$. The fact that this category $L B_{f}$ forms a stack even when $f$ is not represented by a scheme is a preliminary to our compactification. We show that it can be proven by adapting Mumford's treatment of cohomology and base change [Mu70, II. §5] to the stack-theoretic situation: $\mathrm{f}: \mathrm{Y} \rightarrow X$. As mentioned in $\S 3.1$, M. Lieblich provides a more general statement implying this result by showing that the category of flat families of coherent sheaves on $Y$ with proper support over $X$ is an algebraic stack, Li06, Thm. 2.1.1, Lem. 2.3.1].

\section{A.1 The fibred category $L B_{f}$ is a stack}

Let $Y$ be a Deligne-Mumford stack, flat and proper on a base scheme $X$

$$
f: Y \rightarrow X \text {. }
$$

We write $\mathrm{LB}_{\mathrm{f}}$ for the category of line bundles on base changes $\mathrm{Y}_{S}=\mathrm{Y} \times_{X} S$ for every $X$-scheme $S$. More precisely, the objects are pairs $(S, \mathrm{M})$, where $S$ is an $X$-scheme and $\mathrm{M}$ is a line bundle on $\mathrm{Y}_{S}=\mathrm{Y} \times_{X} S$. The morphisms $(S, \mathrm{M}) \rightarrow\left(S^{\prime}, \mathrm{M}^{\prime}\right)$ are pairs $(m, \mathrm{a})$, where $m \in \operatorname{Hom}_{X}\left(S, S^{\prime}\right)$ and $\mathrm{a}$ is an isomorphism of line bundles a: $\mathrm{M} \stackrel{\sim}{\rightarrow} \mathrm{M}^{\prime} \times_{S^{\prime}} S$ on $\mathrm{Y}_{S}$. 
A.1.1 Remark. The category $\mathrm{LB}_{\mathrm{f}}$ induces a functor sending an $X$-scheme $S$ to the groupoid in $\mathrm{LB}_{\mathrm{f}}$ formed by the objects $(S, \mathrm{M})$ on $S$. Even when $\mathrm{f}$ is a representable morphism $f: Y \rightarrow X$, such a functor differs from the functor sending an $X$-scheme $S$ to the $\operatorname{set} \operatorname{Pic}\left(Y_{S}\right)$, which is the functor used in Grothendieck's treatment Gr68] of the relative Picard functor. We illustrate the relation between the two functors at the end of this appendix using the notion of rigidification of a stack along a group scheme, Theorem A.2.1.

A.1.2 Proposition. On a base scheme $X$, let $\mathrm{f}: \mathrm{Y} \rightarrow X$ be a flat and proper morphism of Deligne-Mumford type, with geometrically connected fibres, tame, and coarsely represented by an $X$-scheme $|\mathrm{Y}|$, projective and flat on $X$. Then, the category $\mathrm{LB}_{\mathrm{f}}$ is a stack on $X$.

Proof. Since the category is fibred in groupoids by definition, we only need to show

1. the representability of the isomorphism functors,

2. the effectiveness of any étale descent datum of objects

(as far as $\widetilde{\mathrm{LB}}_{g}$ is concerned, we ignore the issue of geometrization, namely the existence of a smooth and surjective morphism from a scheme to the stack, see Remark A.1.5). We show point (1) by means of the following statement, a stack-theoretic generalization of Mumford's theorem on cohomology and base change [Mu70, II. §5] for schemes.

A.1.3 Lemma. Let $\mathrm{Y}$ be a tame Deligne-Mumford stack, flat and proper over an affine scheme $S$. Assume that the morphism of schemes $|\mathrm{Y}| \rightarrow S$ is flat. Let $\mathcal{E}$ be a locally free and coherent sheaf on $\mathrm{Y}$. Then, there exists a perfect complex $K^{\bullet}: 0 \rightarrow K^{0} \rightarrow K^{1} \rightarrow \cdots \rightarrow K^{n} \rightarrow 0$ on $S$ and an isomorphism of functors

$$
H^{p}\left(\mathrm{Y}_{T}, \mathcal{E}_{T}\right)=H^{p}\left(K_{T}^{\bullet}\right)
$$

on the category of $S$-schemes $T$ (here, $Y_{T}, \mathcal{E}_{T}$, and $K_{T}^{\bullet}$ denote the base change via $T \rightarrow S$ ).

Proof. Recall that the direct image via $\pi: \mathrm{Y} \rightarrow|\mathrm{Y}|$ is an exact functor from the category of coherent sheaves on $\mathrm{Y}$ to the category of coherent sheaves on $|\mathrm{Y}|$, [AV02, Lem.2.3.4]. Therefore, we have the isomorphism $H^{p}\left(\mathrm{Y}_{T}, \mathcal{E}_{T}\right) \cong H^{p}\left(|\mathrm{Y}|_{T}, \pi_{*} \mathcal{E}_{T}\right)$, and it is enough to find a complex $K^{\bullet}$ satisfying

$$
H^{p}\left(|\mathrm{Y}|_{T}, \pi_{*} \mathcal{E}_{T}\right) \cong H^{p}\left(K_{T}^{\bullet}\right)
$$

Mumford' theorem [Mu70, II. §5] shows that such a $K^{\bullet}$ exists if $\pi_{*} \mathcal{E}$ is a coherent sheaf, flat on $S=\operatorname{Spec} A$. Indeed, we check that $\pi_{*} \mathcal{E}$ is flat on $S$, which means that, locally on $|\mathrm{Y}|$, there exists an affine open set on which $\pi_{*} \mathcal{E}$ is given by a flat $A$-module. This happens because, as shown in [AV02, Lem.2.2.3], for a stack $\mathrm{Y}$ of Deligne-Mumford type, there is an étale covering $Y_{\alpha} \rightarrow|\mathrm{Y}|$ such that, for all $\alpha$, the pullback $\mathrm{Y} \times_{|\mathrm{Y}|} Y_{\alpha}$ is a quotient stack of the form $\left[U_{\alpha} / G_{\alpha}\right]$, where $U_{\alpha}$ is a scheme and $G_{\alpha}$ is a finite group acting on $U_{\alpha}$ (note that since $\mathrm{Y}$ is tame, \# $\left(G_{\alpha}\right)$ is prime to the residue characteristic). So, on an affine open set $V \subset|\mathrm{Y}|, \pi_{*} \mathcal{E}$ can be regarded as the direct image of a locally free $G$-equivariant coherent sheaf on an affine scheme $U=\operatorname{Spec} R$. Such a sheaf can be regarded as a $G$-linearized $R$-module $M$. Therefore, as an $\mathcal{O}_{V}$-module, $\pi_{*} \mathcal{E}$ corresponds to $M^{G}$, the submodule of $M$ of $G$-invariant elements. The tameness assumption implies linear reductiveness: $M$ splits as $M^{G} \oplus M^{\prime}$. So, as an $A$-module, $M^{G}$ is flat, because it is a direct summand of $M$ and, on the other hand, $M$ is flat over $R$, which is flat over $A$. 
Now, point (1) follows from the following lemma.

A.1.4 Lemma. For any scheme $S$ and objects $\alpha=\left(S, \mathrm{M}_{\alpha}\right)$ and $\beta=\left(S, \mathrm{M}_{\beta}\right)$ in $\mathrm{LB}_{\mathrm{f}}$ the functor $\operatorname{Isom}_{S}(\alpha, \beta)$ from $S$-schemes to sets is represented by a separated scheme locally of finite type over $S$.

Proof. Write $\mathrm{D}_{S}=\mathrm{M}_{\alpha} \otimes \mathrm{M}_{\beta}^{\vee}$. We write $\pi$ for $\mathrm{Y} \rightarrow|\mathrm{Y}|$, and we adopt the notation $\mathrm{p}: \mathrm{Y}_{S} \rightarrow S$ and $p:|\mathrm{Y}|_{S} \rightarrow S$. For any morphism $Z \rightarrow S$ write $\mathrm{Y}_{Z}$ and $\mathrm{D}_{Z}$ for the base change of $\mathrm{Y}_{S}$ and $\mathrm{D}_{S}$. We need to represent the functor $\operatorname{Isom}_{S}(\alpha, \beta)$ sending an $S$-scheme $Z$ to the set of isomorphisms between $\mathrm{M}_{\alpha}$ and $\mathrm{M}_{\beta}$ on $Z$. This is equivalent to the set of nonzero sections $s \in \Gamma\left(\mathrm{Y}_{Z}, \mathrm{D}_{Z}\right)$, where $s$ is nowhere vanishing. By Lemma A.1.3, we can take $d: K^{0} \rightarrow K^{1}$, a homomorphism of vector bundles on $S$; then, the $S$-scheme $T_{\alpha, \beta}=\{d=0\}$ of $K^{0}$ represents represent the functor $\operatorname{Hom}_{S}(\alpha, \beta)$ sending an $S$-scheme $Z$ to the set of homomorphisms between $\mathrm{M}_{\alpha}$ and $\mathrm{M}_{\beta}$ on $Z$. The composition of homomorphism induces a morphism

$$
c: T_{\alpha, \beta} \times_{S} T_{\beta, \alpha} \rightarrow T_{\alpha, \alpha} .
$$

Let $1: S \rightarrow T_{\alpha, \alpha}$ be the section representing the identity homomorphism. The functor $\operatorname{Isom}_{S}(\alpha, \beta)$ is represented by $\{c=1\}$. Since $K^{0}$ is a finite dimensional vector bundle, $T_{\alpha, \beta}$ is separated and of finite type over $S$. It follows that the scheme representing the functor $\operatorname{Isom}_{S}(\alpha, \beta)$ is separated and of finite type (it is closed in the scheme $T_{\alpha, \beta} \times{ }_{S} T_{\beta, \alpha}$, which is separated and of finite type over $S$ ).

Finally, we show point (2): any étale descent datum of line bundles on $\mathrm{Y}$ is effective. Indeed, given an $X$-scheme $S$, an étale cover $\left(S_{\alpha} \rightarrow S\right)$, and objects $\left(S_{\alpha}, \mathrm{M}_{\alpha}\right)$, where $\mathrm{M}_{\alpha}$ is a line bundle on $\mathrm{Y}_{\alpha}=\mathrm{Y} \times{ }_{S} S_{\alpha}$, together with isomorphisms between pullbacks of $\left(S_{\alpha}, \mathrm{M}_{\alpha}\right)$ and $\left(S_{\beta}, \mathrm{M}_{\beta}\right)$ to $S_{\alpha} \times{ }_{S} S_{\beta}$ satisfying the cocycle condition, we claim that these data descend to an object $(S, \mathrm{M})$, where $\mathrm{M}$ is a line bundle on $\mathrm{Y}_{S}$. Indeed $\mathrm{M}$ is defined by étale descent of line bundles and morphisms of line bundles for schemes: for any scheme $T \rightarrow \mathrm{Y}_{S}$ the line bundle $\mathrm{M}_{T}$ on $T$ is induced by descent along the étale cover $\left(\mathrm{Y}_{\alpha} \times{ }_{\mathrm{Y}_{S}} T \rightarrow T\right)$ of the line bundles $\mathrm{M}_{\alpha} \times{ }_{\mathrm{Y}_{S}} T$ on $\mathrm{Y}_{\alpha} \times \mathrm{Y}_{S} T$.

A.1.5 Remark. Although we did not show that $\mathrm{LB}_{\mathrm{f}}$ is an algebraic stack in the sense of Artin's definition, we point out that Lemma A.1.4 together with the fact that the relative cotangent complex of $\mathrm{F}^{1 / r} \rightarrow X$ is trivial (see 3.1.3) can be used to prove that the stack $\mathrm{F}^{1 / r}$ is algebraic. The claim follows easily from Artin's method, in which one starts from a deformation theory, constructs formal deformation spaces, and shows that they are algebraizable. Indeed the deformation functor of $\mathrm{F}^{1 / r}$ coincides with that of $X$, since the relative cotangent complex of $\mathrm{F}^{1 / r} \rightarrow X$ is trivial. To finish the proof one only needs to show that formal deformations are algebraizable, which is an immediate consequence of Grothendieck's Existence Theorem extended to tame stacks by Abramovich and Vistoli, AV02.

\section{A.2 Rigidification}

Note that each object $(S, \mathrm{~L})$ of $\mathrm{LB}_{\mathrm{f}}$ over an $X$-scheme $S$ has automorphisms given by multiplication by $s \in \Gamma\left(S, \mathbb{G}_{m}\right)$ along the fibre of $\mathrm{L}$. More precisely, $\mathrm{H}=\mathrm{LB}_{\mathrm{f}}$ and $G=\mathbb{G}_{m}$ fit in the 
following setting.

A.2.1 Theorem (Abramovich, Corti, Vistoli, ACV03, Thm. 5.1.5]). Let $\mathrm{H}$ be a stack on a base scheme $X$, let $G$ be a flat finitely presented group scheme on $X$, and assume that for any object $\tau$ of $\mathrm{H}$ over an $X$-scheme $S$ there is an embedding $i_{\tau}: G(S) \hookrightarrow \operatorname{Aut}_{S}(\tau)$ compatible with pullbacks in the obvious sense (for any $\varphi: \tau \rightarrow \tau^{\prime}$ in $\mathrm{H}$ over the morphism of $X$-schemes $f: S \rightarrow S^{\prime}$ we have $\left.i_{\tau} \circ f^{*}=\varphi^{*} \circ i_{\tau^{\prime}}\right)$. Then, there exists a stack $\mathrm{H}^{G}$ and a morphism of stacks $\mathrm{H} \rightarrow \mathrm{H}^{G}$ over $X$ satisfying the following conditions.

1. For any object $\tau \in \mathrm{H}(S)$ with image $\xi \in \mathrm{H}^{G}(S)$, the set $G(S)$ lies in the kernel of $\operatorname{Aut}_{S}(\xi) \rightarrow$ $\operatorname{Aut}_{S}(\tau)$.

2. The morphism $\mathrm{H} \rightarrow \mathrm{H}^{G}$ is universal for morphisms of stacks $\mathrm{H} \rightarrow \mathrm{H}^{\prime}$ satisfying (1) above.

3. In the condition (1) above, if $S$ is the spectrum of an algebraically closed field, we have $\operatorname{Aut}_{S}(\xi)=\operatorname{Aut}_{S}(\tau) / G(S)$.

If $\mathrm{H}$ is an algebraic stack, then $\mathrm{H}^{G}$ is also an algebraic stack. If $\mathrm{H}$ is of Deligne-Mumford type, then $\mathrm{H}^{G}$ is also of Deligne-Mumford type and the coarse space $|\mathrm{H}|$ is isomorphic to $\left|\mathrm{H}^{G}\right|$.

We call $\mathrm{H}^{G}$ the rigidification of $\mathrm{H}$ along $G$. In [Ro, I. Prop. 3.0.2, (2)], Romagny shows that if $\mathrm{H}$ is a Deligne-Mumford stack, then it is "locally isomorphic" to $\mathrm{B} G$ (on $\mathrm{H}^{G}$ ) and is indeed an étale $G$-gerbe.

The construction of $\mathrm{H}^{G}$ consists of two steps.

1. We define a prestack $\mathrm{H}_{\text {pre }}^{G}$ : the category whose objects are the objects of $H$ and whose morphisms are obtained by means of a quotient operation on the sheaves of morphisms of $\mathrm{H}$ (for any object $\tau$ the embeddings $G(S) \hookrightarrow \operatorname{Aut}_{S}(\tau)$ induce a categorically injective morphism of $S$-group schemes of the pullback $G_{S}$ of $G$ to $S$ to the group scheme $\operatorname{Aut}_{S}(\tau)$ of automorphisms of $\tau$ ).

2. We pass to the stack $\mathrm{H}^{G}$ associated to the prestack $\mathrm{H}_{\text {pre }}^{G}$ in the sense of [LM00, Lem. 3.2].

This construction provides a natural framework to a standard procedure that occurs systematically in the construction of the Picard functor. In general, for any morphism of schemes $f: Y \rightarrow X$, the natural functor $S \mapsto \operatorname{Pic}\left(Y_{S}\right)$ from $X$-schemes to sets is a presheaf and is not represented by a scheme. The actual "relative Picard functor" is defined by the passage to the associated sheaf. This point is illustrated in detail in [BLR80, Ch. 8], by Bosch, Lütkebohmert, and Raynaud. In this way, the construction of the relative Picard functor is just another way to rigidify $\mathrm{LB}_{f}$ along $\mathbb{G}_{m}$.

\section{References}

[Ab] D. Abramovich, Lectures on Gromov-Witten invariants of orbifolds, Preprint: math. AG/0512372.

[ACV03] D. Abramovich, A. Corti, A. Vistoli, Twisted bundles and admissible covers, Communications in Algebra 31 (2003), 3547-3618.

[AGV01] D. Abramovich, T. Graber, A. Vistoli, Algebraic orbifold quantum products. Orbifolds in mathematics and physics (Madison, WI, 2001), Contemp. Math., 310, Amer. Math. Soc., Providence, RI, 2002. 1-24 
[AJ03] D. Abramovich, T. J. Jarvis, Moduli of twisted spin curves, Proc. Amer. Math. Soc. 131 (2003), 685-699.

[AV02] D. Abramovich, A. Vistoli, Compactifying the space of stable maps, J. Amer. Math. Soc. 15 (2002), 27-75.

[AC87] E. Arbarello, M. Cornalba, The Picard groups of the moduli spaces of curves, Topology 26 (1987), 153-171.

[Ar74] M. Artin, Versal deformations and algebraic stacks, Invent. Math. 27 (1974), 165-189.

[BLR80] S. Bosch, W. Lütkebohmert, M. Raynaud, Néron models, Results in Mathematics and Related Areas (3), vol. 21, Springer-Verlag, Berlin, 1990.

[BG] J. Bryan, T. Graber, The Crepant Resolution Conjecture, Preprint: math.AG/0610129.

[Br90] L. Breen, Bitorseurs et cohomologie non-abélienne, The Grothendieck Festschrift, Vol. I, 401-476, Progr. Math., 86, Birkhäuser Boston, Boston, MA, 1990.

[Ca07] C. Cadman, Using stacks to impose tangency conditions on curves, Amer. J. Math. 2007, 129 (2), 405-427. math. AG/0312349

[CCC07] L. Caporaso, C. Casagrande, M. Cornalba, Moduli of roots of line bundles on curves, Trans. Amer. Math. Soc. 359 (2007), 3733-3768. math. AG/0404078

[CCIT] Coates, Corti, Iritani, Tseng, Computing Genus-Zero Twisted Gromov-Witten Invariants, Preprint: math/0702234

[Ch06] A. Chiodo, The Witten top Chern class via K-theory, J. Algebraic Geom., 15, 2006, 681-707. math. AG/0210398.

[Ch1] A. ChIodo, Quantitative Néron theory for torsion bundles, Preprint: math.AG/0603689.

[Ch2] A. ChIODO, Towards an enumerative geometry of the moduli space of twisted curves and $r$-th roots, Preprint: math.AG/0607324.

[Co87] M. Cornalba, Moduli of curves and theta-characteristics, "Lectures on Riemann Surfaces", Ed. M. Cornalba, X. Gomez-Mont, A. Verjovsky, World Scientific, Singapore, (1989), 560-589.

[DM69] P. Deligne, D. Mumford, Irreducibility of the space of curves of given genus, Inst. Hautes Études Sci. Publ. Math. 36 (1969), 75-112.

[FSZ] C. Faber, S. Shadrin, D. Zvonkine, Tautological relations and the $r$-spin Witten conjecture, Preprint: math.AG/0612510

[Gr68] A. GRothENDIECK, Technique de descente et théorèmes d'existence en géométrie algébrique. I-V. Séminaire Bourbaki, Vol. 7, Exp. No. 236, Soc. Math. France, Paris, (1995) 221-243.

[Ha83] J. HARER, The second homology group of the mapping class group of an orientable surface. Invent. Math. 72 (1983), no. 2, 221-239.

[I172] L. ILlusie, Complexe cotangent et déformations. I. Lecture Notes in Mathematics, Vol. 239, Springer-Verlag (1971) xv+355 pp. ; II. Lecture Notes in Mathematics, Vol. 283, SpringerVerlag (1972) vii+304 pp.

[Ja98] T. J. JARVIS, Torsion-free sheaves and moduli of generalized spin curves. Compositio Mathematica, 110 (1998), 291-333.

[Ja00] T. J. JARvis, Geometry of the moduli of higher spin curves, Internat. J. Math. 11 (2000), $637-663$.

[Ja01] T. J. JARvis, The Picard group of the moduli of higher spin curves, New York Journal of Mathematics, 7, (2001), 23-47.

[JKV01] T. J. Jarvis, T. Kimura, A. Vaintrob, Moduli spaces of higher spin curves and integrable hierarchies. Compositio Math. 126, (2001), no. 2, 157-212. 
[JKV00] T. J. Jarvis, T. Kimura, A. Vaintrob, Tensor products of Frobenius manifolds and moduli spaces of higher spin curves, Conférence Moshé Flato 1999, Vol. II (Dijon), Math. Phys. Stud., 22, Kluwer Acad. Publ., Dordrecht, (2000), 145-166.

[JKV05] T. J. Jarvis, T. Kimura, A. Vaintrob, Stable spin maps, Gromov-Witten invariants, and quantum cohomology, Commun. Math. Phys. 259(3), 511-543 (2005).

[Ka89] L. Kato, Logarithmic structures of Fontaine-Illusie, Algebraic analysis, geometry, and number theory Johns Hopkins Univ. Press, Baltimore, MD, (1989), 191-224.

[Ko92] M. Kontsevich, Intersection theory of the moduli space of curves and the matrix Airy function. Comm. Math. Phys. 147 (1992), no. 1, 1-23.

[Kr99] A. Kresch, Cycle groups for Artin stacks, Invent. Math. 138 (1999), 495-536.

[KM97] S. KeEL, S. MorI, Quotients by groupoids. Ann. of Math. (2) 145 (1997), no. 1, 193-213.

[LM00] G. Laumon, L. Moret-Bailly, Champs algébriques, Springer Verlag, Berlin (2000), xii +208 .

[Li06] M. LieBLich, Remarks on the stack of coherent algebras, Int. Math. Res. Not., pages Art. ID $75273,12,2006$.

[Me87] N. Mestrano, Conjecture de Franchetta forte. Invent. Math. 87 (1987), 365-376.

[Mi80] J. Milne, Étale cohomology. Princeton Mathematical Series, 33. Princeton University Press, Princeton, 1980.

[MO05] K. Matsuki, M. Olsson, Kawamata-Viehweg vanishing and Kodaira vanishing for stacks Math. Res. Letters 12 (2005), 207-217.

[Mu70] D. Mumford, Abelian varieties, Tata Institute of Fundamental Research Studies in Mathematics, No. 5, Bombay, 1970.

[O107] M. Olsson, On (log) twisted curves, Compositio Mathematicae, 143 (2007), 476-494. Preprint version: http://www.ma.utexas.edu/molsson/Logcurves.pdf

[PV01] A. Polishchuk, A. Vaintrob, Algebraic construction of Witten's top Chern class in "Advances in algebraic geometry motivated by physics", (Lowell, MA, 2000), 229-250, Contemp. Math. 276, Ed. E. Previato, AMS, Providence, RI, (2001).

[Ra70] M. Raynaud, Spécialisation du foncteur de Picard. Inst. Hautes Études Sci. Publ. Math. No. $38197027-76$.

[Ro] M. Romagny, Sur quelques aspects des champs de revêtements de courbes algébriques, Ph.D. Thesis, 2002, 120. http://www-fourier.ujf-grenoble.fr/THESE/ps/t120.ps.gz

[Wi91] E. WitTen, Two-dimensional gravity and intersection theory on moduli space, Surveys in differential geometry, (Cambridge, MA, 1990), 243-310, Lehigh Univ., Bethlehem, PA, (1991).

[Wi93] E. WitTen, Algebraic geometry associated with matrix models of two-dimensional gravity, "Topological methods in modern mathematics" (Stony Brook, NY, 1991) Publish or Perish, Houston, TX (1993), 235-269. 